University of Louisville

ThinkIR: The University of Louisville's Institutional Repository

Electronic Theses and Dissertations

$12-2018$

\title{
Low temperature desiccants in atmospheric water generation.
}

Sunil Gupta

University of Louisville

Follow this and additional works at: https://ir.library.louisville.edu/etd

Part of the Applied Mechanics Commons, Energy Systems Commons, Heat Transfer, Combustion Commons, Nanoscience and Nanotechnology Commons, Other Chemical Engineering Commons, and the Other Materials Science and Engineering Commons

\section{Recommended Citation}

Gupta, Sunil, "Low temperature desiccants in atmospheric water generation." (2018). Electronic Theses and Dissertations. Paper 3087.

https://doi.org/10.18297/etd/3087

This Master's Thesis is brought to you for free and open access by ThinkIR: The University of Louisville's Institutional Repository. It has been accepted for inclusion in Electronic Theses and Dissertations by an authorized administrator of ThinkIR: The University of Louisville's Institutional Repository. This title appears here courtesy of the author, who has retained all other copyrights. For more information, please contact thinkir@louisville.edu. 
LOW TEMPERATURE DESICCANTS IN ATMOSPHERIC WATER GENERATION

By

Sunil Gupta

\author{
A Thesis \\ Submitted to the Faculty of the \\ J.B. Speed School of Engineering \\ In Fulfillment of the Requirements \\ For the Degree of \\ Master of Science \\ in Mechanical Engineering
}

Department of Mechanical Engineering

University of Louisville

Louisville, KY 40292

December 2018 

Sunil Gupta

A 7 KHMV Approved on

July 26, 2018

by the Following Thesis Committee

Dr. Ellen Brehob (Thesis Director)

Dr. Srinivasan Rasipuram (Committee Member)

Dr. William Mark McGinley (Committee Member)

Dr. Thad Druffel (Committee Member) 


\section{ACKNOWLEDGEMENTS}

I acknowledge and thank my thesis committee for all of their assistance in directing this study and in the writing of this thesis, namely Dr. Brehob, Dr. Rasipuram, Dr. McGinley and Dr. Druffel.

Dr. Druffel was my chief mentor for the duration of this research, and his guidance was invaluable - from core mechanical, thermal \& electrical elements of the prototype to material synthesis, characterization and experimental setup on the desiccant research.

I also thank all those who helped in accomplishing this work, Brianna Price \& Dhruv Patel, both of whom undertook their Co-op on this research; several students, notably Peter Heiniger \& David Bergman, who worked with me on the first prototype for their capstone project; Pranav Singh \& Harshit Mishra, interns; Juan He from Advanced Energy Materials; Veerandra Alta, PhD candidate, and Dr. Sunkara, Conn Center. Special thanks for the feedback and assistance from my research group.

It was a pleasure to work with everyone. 
Finally, I thank my immediate family, Anjana, Shruthi \& Karan, all in India, for supporting me in this endeavor and for the encouragement through a new and wonderful process of re-learning!

Sunil Gupta 


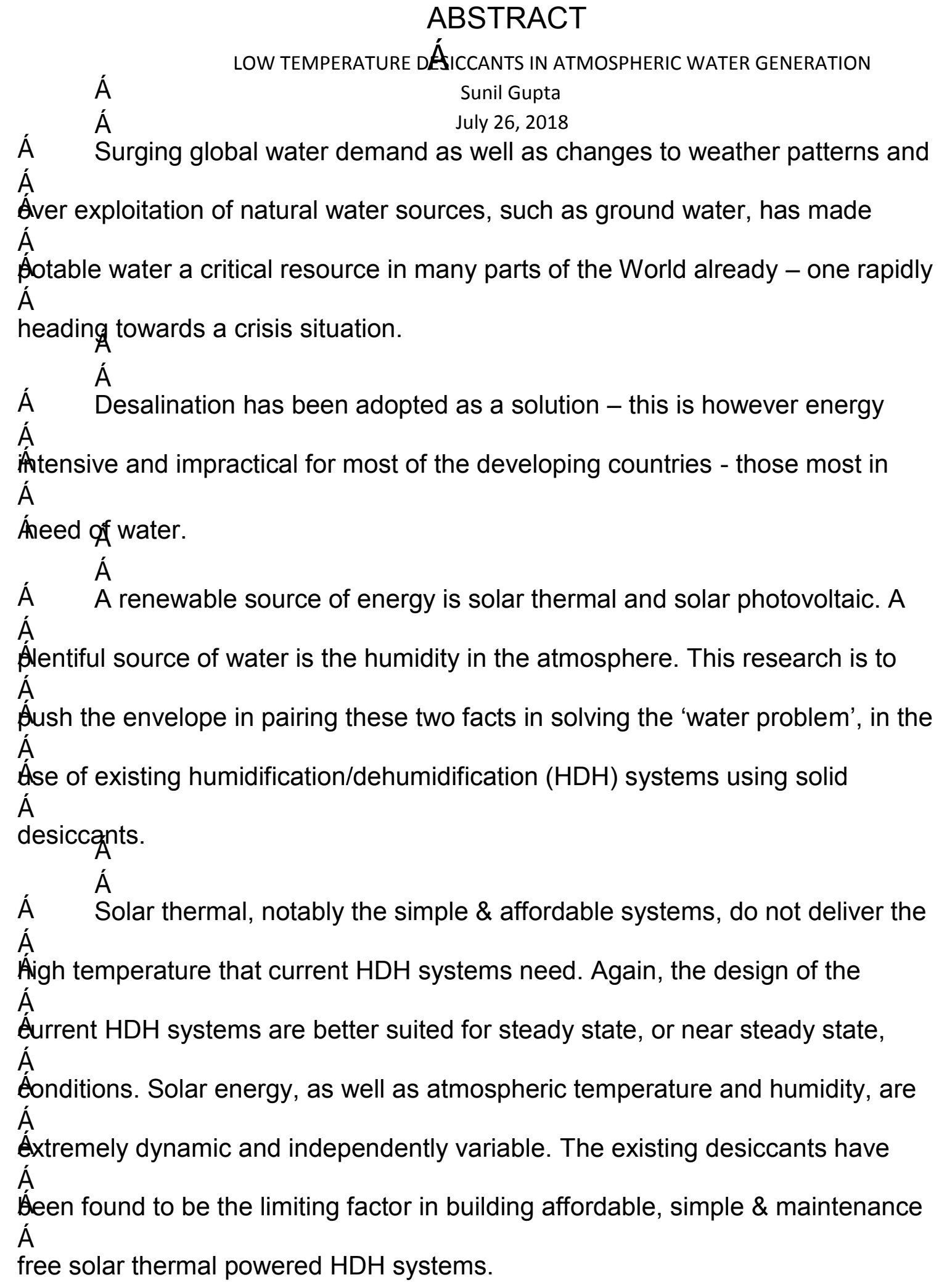


The result of this study identifies a promising new lithium oxide nano material that meets or exceeds the specifications for such a desiccant. Specifically, Lithium Aluminate $\left(\mathrm{LiAlO}_{2}\right)$ nano powder, synthesized using a UofL patented process, was found to be have ultra-fast kinetics (of the order of 2-5 minutes for adsorption as well as desorption), low temperature desorption (60$\left.80^{\circ} \mathrm{C}\right)$ and low cost $(\$ 20 / \mathrm{kg})$.

As a spin-off benefit, a validated system (described in Appendix 2) was also built that handles the dynamic environmental factors that such an $\mathrm{HDH}$ system will need to manage in real-world installations. 


\section{TABLE OF CONTENTS}

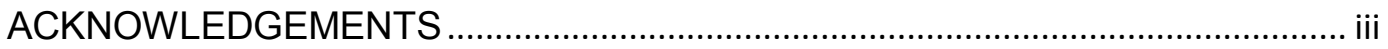

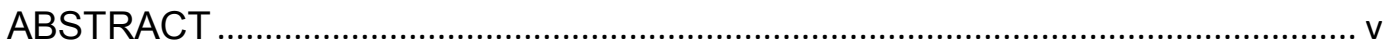

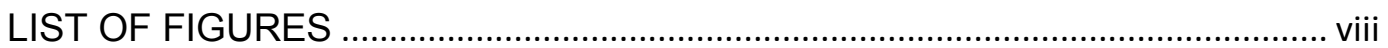

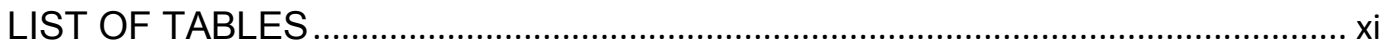

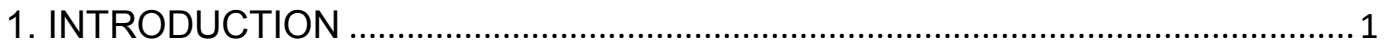

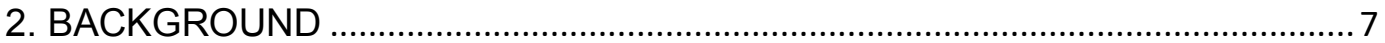

3. SCOPE

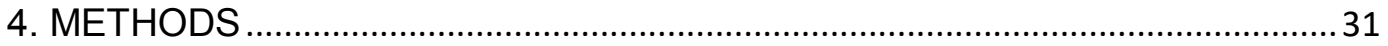

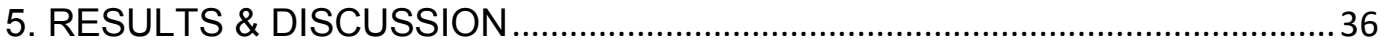

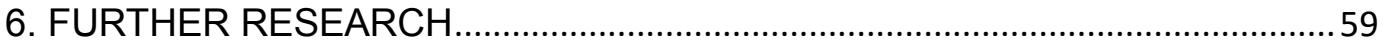

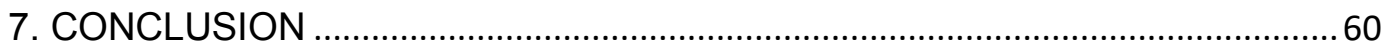

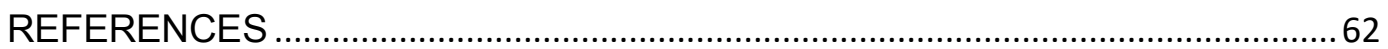

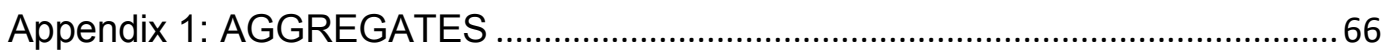

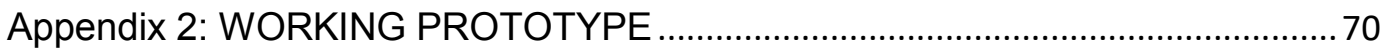

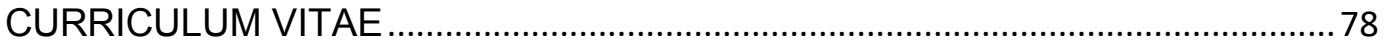




\section{LIST OF FIGURES}

Figure 1: Types of Atmospheric Water Generators [14] ................................................

Figure 2: Design Type 1: Simple condensation of humidity in air [17] ...............................

Figure 3: Design Type 2: Adsorb/Desorb - a coated desiccant wheel [39] ........................ 4

Figure 4: Air Moisture Holding Capacity. Graph shows how capacity increases

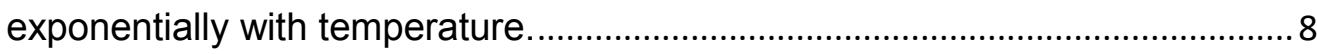

Figure 5: Condensation by cooling. Air at $35^{\circ} \mathrm{C}$ and $60 \% \mathrm{RH}$ will have to be cooled to

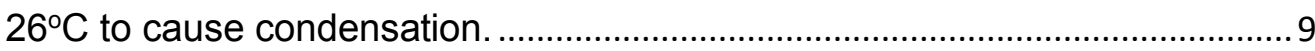

Figure 6: Typical adsorb/desorb atmospheric water generator. Ambient air is the source of moisture in the adsorb phase. Ambient air is the sink to condense the hightemperature air stream after picking up moisture from the desiccant. .10

Figure 7: Desiccant Capacity [55]. Different desiccants have markedly different capacities at different temperatures.

Figure 8: Water Vapor Adsorption Isotherm [62]. The Langmuir isotherm is ideal for our requirements as a desiccant with this behavior shows a uniform capacity after a minimum humidity level. 14

Figure 9: Vapor Pressure vs Moisture [55]. The stages of Adsorption, Desorption and Cooling of a desiccant, along with its moisture content.

Figure 10: Airflow temperature and humidity changes [55] in a rotary HDH system, showing the temperature and moisture changes over the Adsorption and Desorption stages.......

Figure 11: Psychrometric chart [36] illustrating the humidity ratio (water content) of air in the dehumidification loop. 20 
Figure 12: TGA for a LAO sample showing the point of maximum heat flow (representing the desorb temperature) and the maximum desiccant capacity.

Figure 13: A typical TGA-DSC curve for dehydration of $\mathrm{MgSO}_{4} \cdot 7 \mathrm{H}_{2} \mathrm{O}$. The blue line denotes TGA curve and the red line denotes the DSC curve. .23

Figure 14: Desalination design for maximum GOR - showing a stratified design. .24

Figure 15: Desiccant Cooling System [71], using a heat recovery wheel. The colored lines in (b) correspond to the stages in (a). .25

Figure 16: SEM of sol-gel synthesized LAO showing nano rods. .37

Figure 17: TGA of sol-gel LAO that shows a marked inverse peak in the heat flow ........39

Figure 18: TGA of LAO from 1:3 molar pre-cursor mix that does not have a characteristic peak .42

Figure 19: TGA of LAO from 1:5 molar pre-cursor mix showing the inverse peak at $58^{\circ} \mathrm{C}$

Figure 20: TGA of LAO from 1:6 molar pre-cursor mix showing the inverse peak at $68^{\circ} \mathrm{C}$

Figure 21: TGA of LAO from 1:7 molar pre-cursor mix showing the inverse peak at $72^{\circ} \mathrm{C}$

Figure 22: TGA of partially washed 1:6 nano LAO (typical results) showing the inverse peak at $35^{\circ} \mathrm{C}$ and $<2 \%$ desorption

Figure 23: TGA of twice washed 1:6 nano-LAO, vacuum dried, that does not show the characteristic desiccant curves

Figure 24: TGA of single washed $1: 6$ nano-LAO, dried at $120^{\circ} \mathrm{C}$, that does not show the characteristic desiccant curves. .45

Figure 25: Proposed direct synthesis of LAO on metal foil where the sheet is coated in a dip tray and immediately subject to plasma oxidation .47 
Figure 26: SEM of on-aluminum synthesized LAO showing nano fibres 48

Figure 27: Adsorption vs Coating Thickness, with error bars.

49

Figure 28: TGA: On-glass synthesis with TritonX-114, + sinter @ 400C showing 12\% desorption capacity at $70^{\circ} \mathrm{C}$ .51

Figure 29: XRD of LAO coatings, sintered @ $550^{\circ} \mathrm{C}$ that show chemical changes due to the process employed 52

Figure 30: XRD of LAO coatings, sintered @ $900^{\circ} \mathrm{C}$ also showing chemical changes....52

Figure 31: Dried extrudates, before sintering .67

Figure 32: Sintered Extrudates 67

Figure 33: Test rig for the extrudates, using a modified syringe .68

Figure 34: Typical TGA of washed sample showing a marked reduction of the desiccant's capacity 69

Figure 35: Schematic of the prototype 70

Figure 36: Solid model of prototype 72

Figure 37: Prototype fabrication in progress...... .73

Figure 38: Use of a car intercooler as the condenser .74

Figure 39: Water collection over time from the prototype using Silica Gel 76 


\section{LIST OF TABLES}

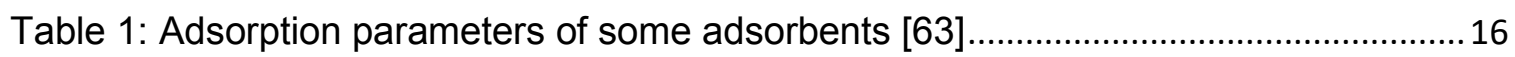

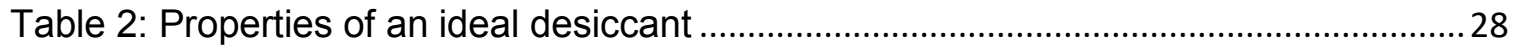

Table 3: TGA summary of different pre-cursor molar mixes ........................................... 41

Table 4: Direct synthesis on aluminum foil, $11.5 \mathrm{~g} / \mathrm{m}^{2}, 120 \mathrm{sec}$ adsorption $+120 \mathrm{sec}$ desorption @ $86^{\circ} \mathrm{C}$............................................................................................ 48

Table 5: Direct synthesis on aluminum foil, $2 \mathrm{~g} / \mathrm{m}^{2}, 120 \mathrm{sec}$ adsorption $+120 \mathrm{sec}$ desorption @ 86 C

Table 6: Time taken for adsorption $(75 \% \mathrm{RH}, 25 \mathrm{C})$ [76] showing the extremely favorable kinetics of LAO vs some commercial desiccants .50

Table 7: Summary of results, as compared to the ideal desiccant....................................55

Table 8: Thermal Efficiency LAO vs Silica Gel, showing 43\% improvement in energy efficiency using LAO. .57

Table 9: Typical results of extrudates using the test rig of Fig 33 with 5 minutes adsorb \& 5 minutes desorb @ $100^{\circ} \mathrm{C}$, showing that the desiccant was destroyed .68

Table 10: Computation for the prototype, for 60 liters/day output, using electricity from the grid. 


\section{INTRODUCTION}

Water scarcity is so pervasive today that solving it is one of the identified goals by the United Nations [1]. Technology development over the years has resulted in the development of industrial water systems like desalination and recovery from waste water, largely using reverse osmosis or multi-stage flash techniques [2]. These plants cater to the needs of cities, and even countries [3]. These are however extremely large-scale projects, requiring a large amount of power and capital outlay, that few countries can muster. As the vast majority of humanity is in developing and third world countries, and they cannot possibly consider these expensive technologies, at least not in the near term. These plants also have a large carbon footprint and negative environmental impact [4].

To illustrate a real-world problem, let us take a very typical situation in India - a group of villages and towns in the northern part of the State of Karnataka. This area frequently suffers draught [5], is far away from the ocean or rivers [6], and has no sources of water other than ground water, which has also become unviable [7]. As a result, this area is one of the more underdeveloped parts of India, resulting in hundreds of farmers committing suicide [8] each year. The agrarian economy in many parts of the World, especially India, is based on rains. Draught resistant plant varieties notwithstanding, often the only thing that differentiates crop failure and success is the availability of just enough water to 
keep the crops alive long enough till the rains come. This is accomplished with techniques like drip-irrigation [9] and hydroponics [10], which have orders of magnitude lower water requirements than traditional farming. Yet, where is even this limited water to come from?

One source of fresh water is the humidity in air, which has been estimated to be ten times the water available in all river systems [11]. Many research groups [12] and several industries [13] are taking advantage of this. Figure 1 broadly illustrates the methods of extraction. The first two, Design Type $1 \& 2$, require energy input, and are discussed in more detail.

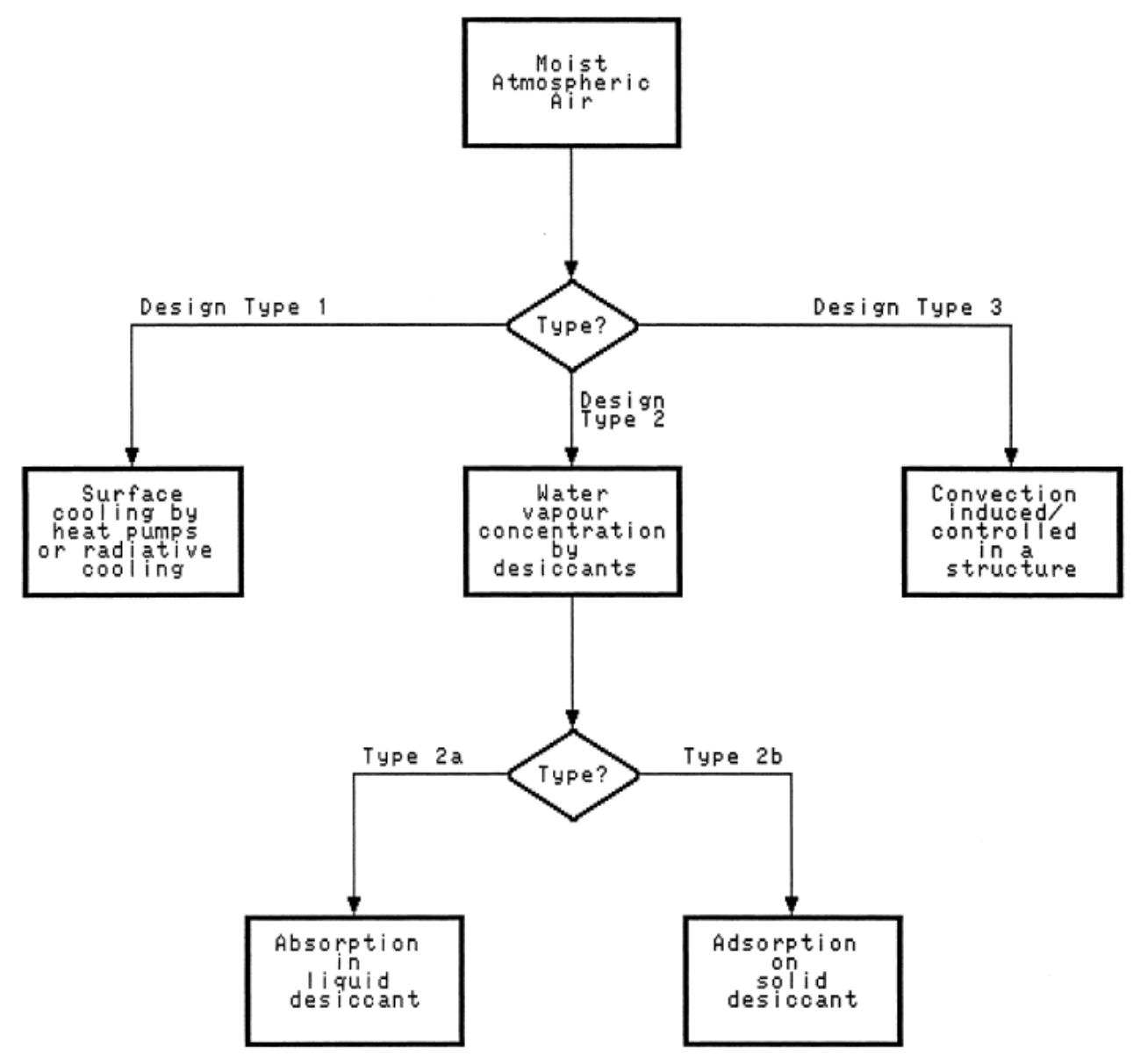

Figure 1: Types of Atmospheric Water Generators [14] 
In the first type, illustrated in Fig 2, humid air is passed over a chilled surface or expanded to produce cooling [15], and the condensate collected. From an energy perspective this method is the least attractive, as energy losses are high and the Gained Output Ratio (GOR) of the system is extremely low. The main advantage of this system is the relative simplicity, which is why this approach is used in common domestic dehumidifiers [16].

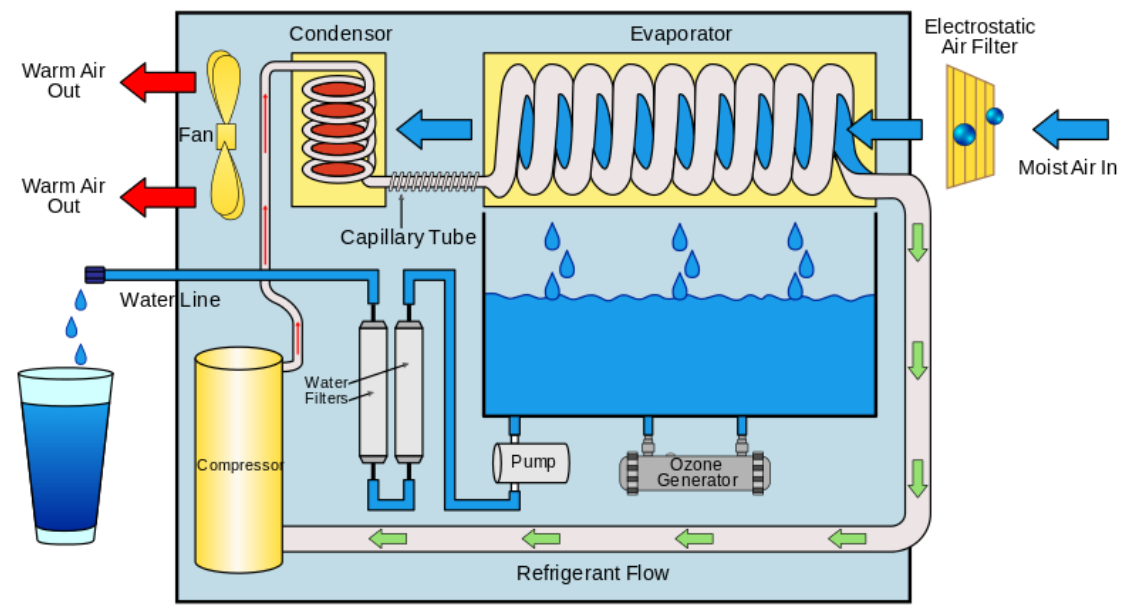

Figure 2: Design Type 1: Simple condensation of humidity in air [17]

The second type, humidification/ dehumidification (HDH), is employed for larger scale systems. Typical HDH systems are composed of three sub-systems 1) humidifier, 2) dehumidifier and 3) heater which are connected in series. The performance is evaluated by the ratio of heat of vaporization $\left(\dot{\mathrm{m}}_{\mathrm{wh}} \mathrm{h}_{\mathrm{fg}}\right)$ to heat input (Qin), represented by the dimensionless number, GOR, which typically ranges from 2-5, where higher GOR values are better [18-22]. The use of solar thermal heat can theoretically increase the GOR of the system up to 12[23], a number demonstrated by several theoretical studies [24-30]. Schemes to increase the 
GOR above 100 requires balancing the thermodynamics between the humidification and dehumidification stages using multi-extraction techniques [3133].

Typically, in these systems, atmospheric air is sent through a solid desiccant packed bed [34-37] as illustrated in Figure 3, or a liquid desiccant [38]. The adsorbed water is then extracted by a high temperature stream of air (Heated Reactivation Air). The resulting air (Wet Air Discharge) is at a high relative humidity $(\mathrm{RH})$, which is then cooled below its dew point and the condensate collected [39]. The literature [19, 24, 28, 32] covers the thermodynamics in great detail.

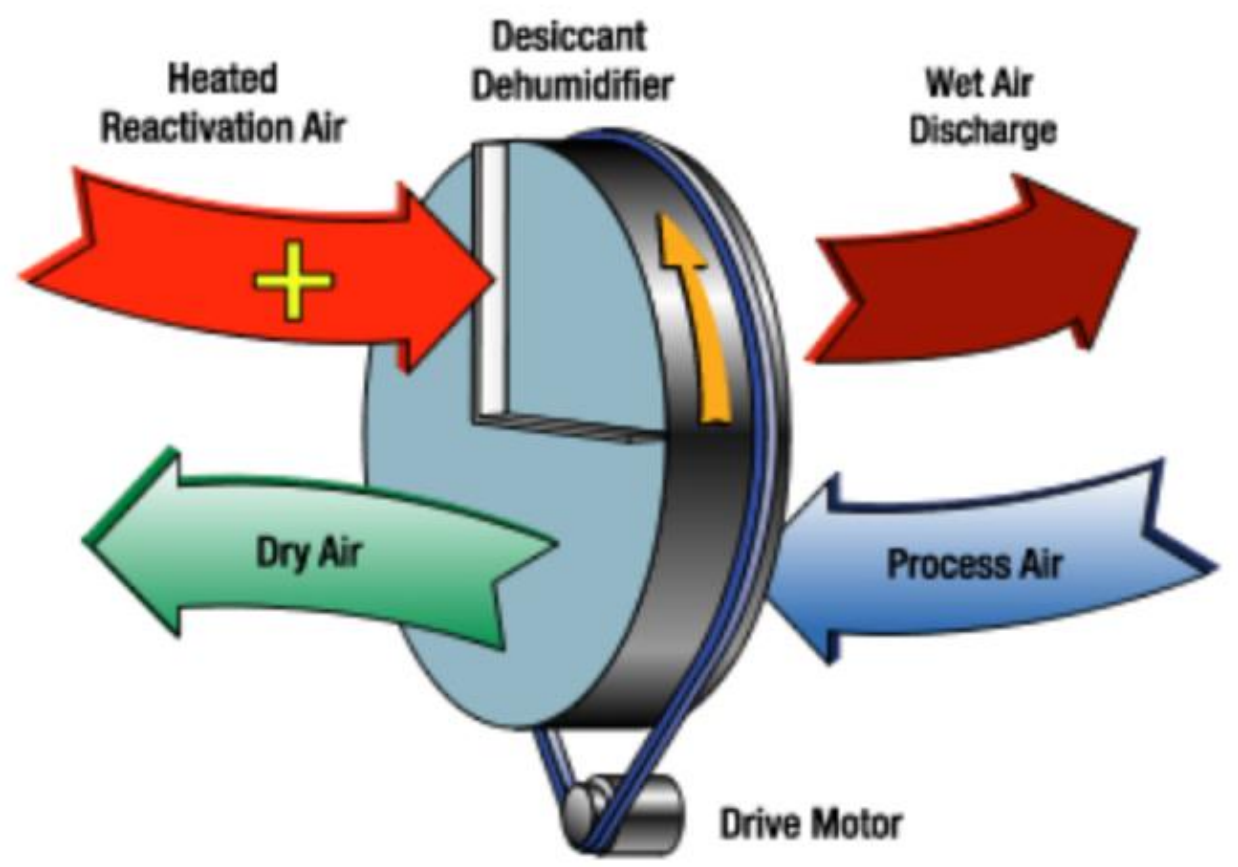

Figure 3: Design Type 2: Adsorb/Desorb - a coated desiccant wheel [39] 
The reason these systems are not in commercial use (other than in situations, like the military, where cost is not a factor) is two-fold: the availability of electric power and the cost of water produced. Commercial Atmospheric Water Generators (AWG) typically [40] require a connected power supply of $20 \mathrm{~kW}$, 3phase, for just 1,000 liters per day, peak. In most developing nations, neither will utility companies supply the required power, nor is the cost of water produced affordable even for domestic use (we can ignore the challenge of capital costs for now, as one can argue that these are issues for economists and policy makers).

Traditional desiccant systems such as silicates (like silica gel) have very slow kinetics and require relatively high temperatures (Table 1) to desorb the water $[41,42]$. Despite this limitation these have been installed in solar cooling systems [43] for a number of years, and recently investigated as a means to produce water from humid atmospheric air [34]. Newer materials such as halide salts and metal organic frameworks (MoF) have pushed the capacities to more than $100 \%$ of the weight of the desiccant; however, the costs and scalability of these are questionable $[35,44]$.

Both these existing characteristics (slow kinetics and high desorption temperatures) of commercially available desiccants limit solar-thermal applications due to the fact that the number of cycles of adsorb/desorb is limited to one per day (the cooler higher $\mathrm{RH}$ night air is suitable for adsorption, while the hot day is used to obtain the high temperature needed for desorption), and the overall system losses due to operating at a high temperature are large, implying a low efficiency. 
What we need are smaller and simpler systems. Smaller systems are possible if the desiccant can be cycled multiple times per day - this needs fast kinetics: adsorption and desorption in minutes and not hours. Simple systems are a possibility with flat-plate air heaters - these however provide air temperatures of $<90^{\circ} \mathrm{C}$ in practice - which means we need a desiccant with a desorption temperature in the $60-80^{\circ} \mathrm{C}$ range. While other aspects of an ideal desiccant are discussed later, these two emerged as critical to our requirements of a desiccant for a sustainable and user-managed solar powered AWG.

We take a detailed look at the literature on various water-from-air systems in Chapter 2, with a focus on desiccants and a review of the available thermodynamic analyses of these systems. Chapter 3 details the scope of this research. Chapter 4 describes the experimental setup. Chapter 5 details the analysis of the new desiccant. Chapter 6 discusses further research that is needed. The conclusions follow in Chapter 7.

Appendix 1 discusses the attempts to develop an aggregate form of the desiccant. Appendix 2 details the specifics of an open-air adsorption, closed-air desorption system, detailing how the dynamic configuration of the uncoupled system offers several advantages over the state of the art. 


\section{BACKGROUND}

Nature incorporated the use of moisture in air as part of survival strategies during evolution of life on Earth. Organisms such as cacti and desert beetles can survive in arid environments because they've evolved mechanisms to collect water from air [45].

Mankind has been able to use simple techniques to condense saturated air, notably at high altitudes with simple mesh systems [46]. These are limited to specific geographies and are best labelled as fog collectors - inexpensive, zero power, with little or no maintenance. Variations of this concept are seen in simple passive devices, like ancient air wells discovered by Beysons, et al., [47] to bamboo towers (convection induced [14]) that several non-governmental organizations are installing across Africa [48] - however, where these work, they are limited to a few liters per day and have no mass potential. Other approaches have used the ground as a thermal sink to condense water [49] - again these approaches have proven impractical as the ground is a poor conductor of heat, and the sink temperature rapidly approaches ambient.

This science is based on the dew point temperature [50]. The humidity tables [51], Fig 4, show that the capacity of air to hold water decreases with decreasing temperature. When we cool air, this causes its relative humidity $(\mathrm{RH})$ to go up, and at the dew point 'drops' the excess water. Fig 5 illustrates how this 
capacity is used to cause condensation. The red arrow on chart shows that air at $35^{\circ} \mathrm{C} 60 \% \mathrm{RH}$, needs to be cooled below $27^{\circ} \mathrm{C}$ for condensation to occur.

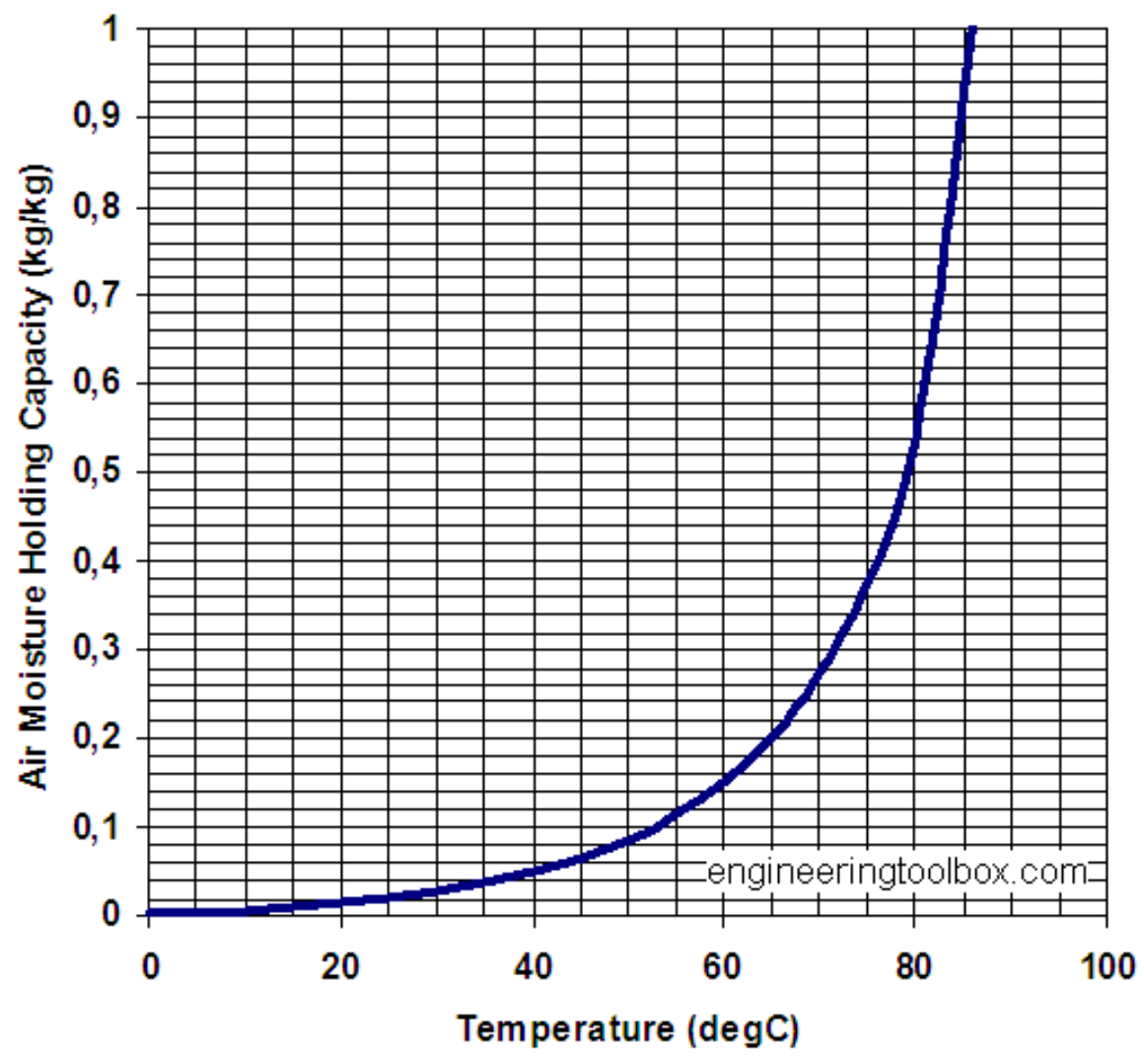

Figure 4: Air Moisture Holding Capacity. Graph shows how capacity increases exponentially with temperature. 


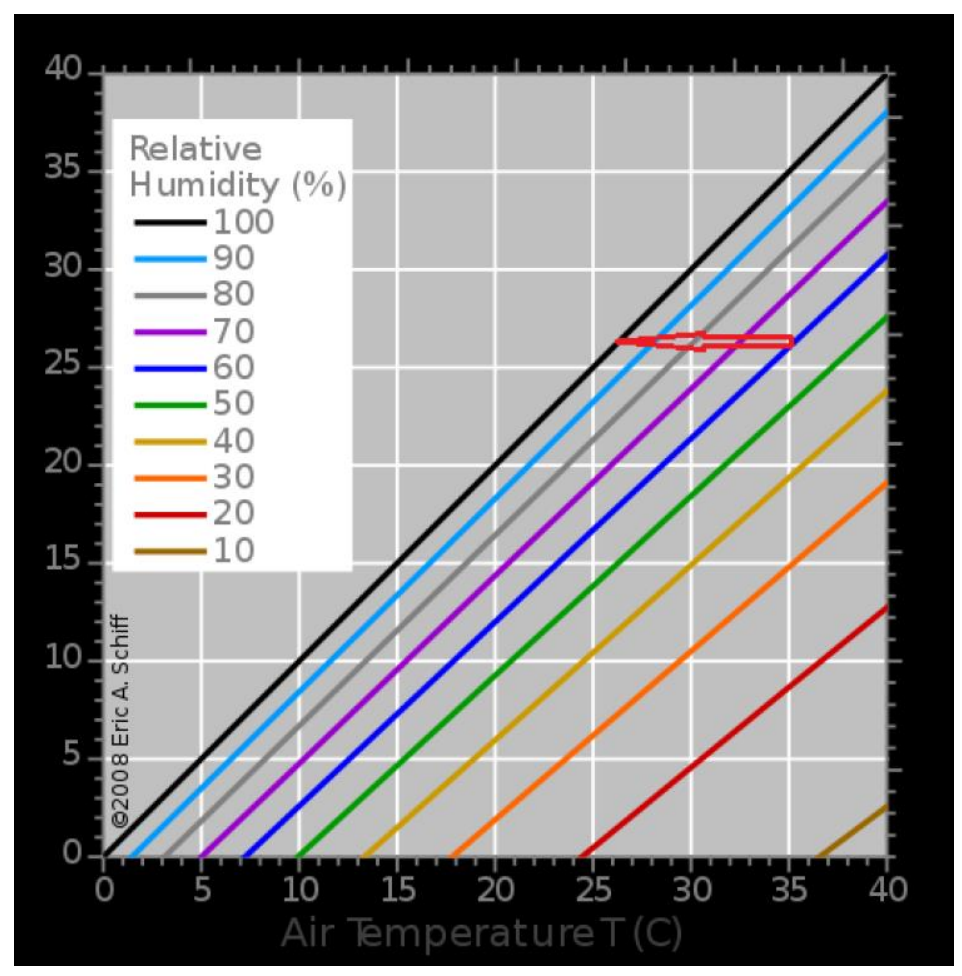

Figure 5: Condensation by cooling. Air at $35^{\circ} \mathrm{C}$ and $60 \% \mathrm{RH}$ will have to be cooled to $26^{\circ} \mathrm{C}$ to cause condensation.

Several AWG systems operate on the 'brute-force' cooling technique

shown in Fig 1. The higher operating temperature is ambient, and the lower is the cooled surface. From an energy perspective, this process has very poor efficiency as there is no possibility to reuse any of the heat produced from the compressor or the condenser. Another disadvantage is that these systems work best when the $\mathrm{RH}$ is above $60 \%$. The higher the $\mathrm{RH}$, the less cooling is required for condensation to occur, and vice-versa: for air at $35^{\circ} \mathrm{C}, 20 \% \mathrm{RH}$, it would need to be cooled down to below $8^{\circ} \mathrm{C}$, to get any condensate. These systems therefore have limited applications, one example being survival, using solar photovoltaics (PV) and Peltier cells for cooling [52, 53]. Due to these limitations, there are no known commercially successful large-scale products using this method. 
The desiccant based approach with its many variations, are energy efficient and even have been successfully applied for commercial products. At a fundamental level, the principle is the same as the previously discussed system, that of condensation. The difference with this system is that humidity is carried by hot air, which holds more water, and the moist air can be cooled below its dew point temperature using ambient air (or sea water/brine). Furthermore, heat recovery sub-systems can add to overall system efficiency.

During adsorption, the latent heat of vaporization is released. This makes the exit air (A), Fig 6, from desiccant (which is in the adsorb phase) hot and dry. During desorption the exit air (B) is cooler, as it has given up heat needed for evaporation. The exit air (C) from the condenser is at near ambient temperature - it is this air that is recirculated to the heater to form a closed air loop.

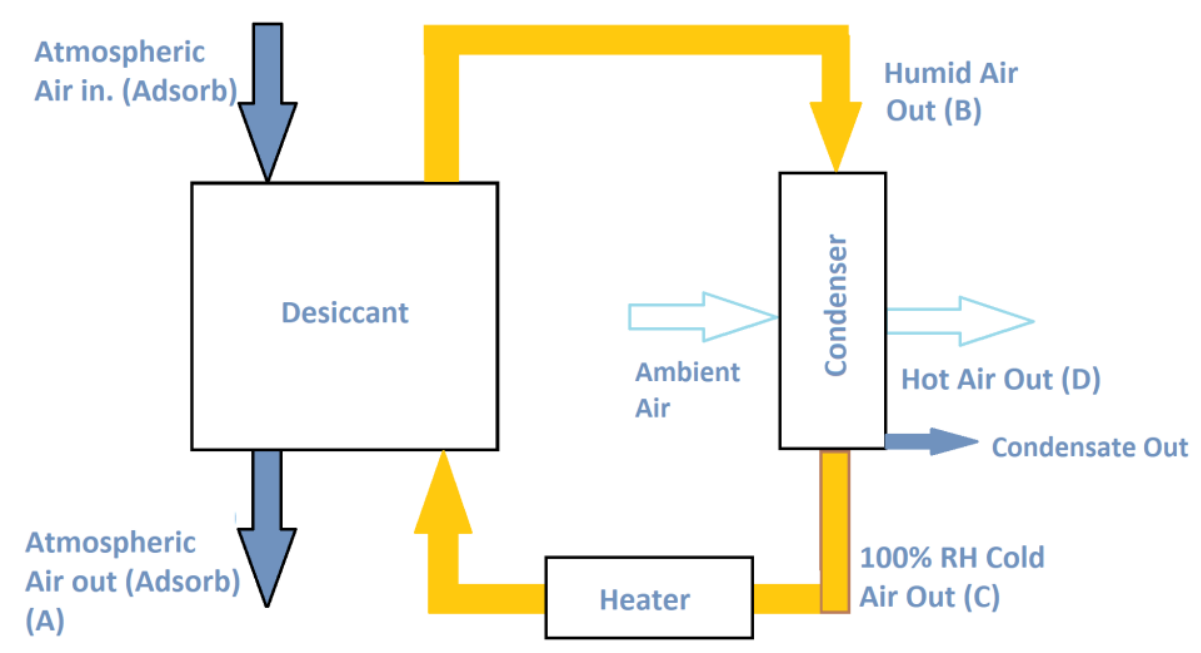

Figure 6: Typical adsorb/desorb atmospheric water generator. Ambient air is the source of moisture in the adsorb phase. Ambient air is the sink to condense the high-temperature air stream after picking up moisture from the desiccant. 
Ambient air, used to cool the condenser, leaves at a higher temperature (D). There is a possibility to reuse some of the heat available at A and D to add to air stream C, to improve energy efficiency.

The same principle is used in desalination or water recovery from industrial waste. These systems can be considered to have just one significant design difference with AWGs - the source of moisture. In the case of AWG, this is moisture from ambient air. In desalination etc., moisture is picked up from the water source by bubbling air through it, heating the water and/or spray drying techniques. Once we have air with high humidity, the process is the same as described for AWG. Typically, referring to Fig 6, incoming feed water is used as the coolant in the condenser (instead of ambient air), thus improving thermal efficiency, and the resulting hotter feed water is evaporated in a stream of air to create the "Atmospheric Air In". As these systems have different thermodynamics from AWG, we will not discuss these in any more detail. Detailed reviews of these systems is available in the literature [54], as well as extensive cost analysis [14].

Irrespective of the specific desiccant used, the following parameters are critical in the design of an optimal system:

- Adsorption capacity: this is a measure of the water-holding capacity of the desiccant. Ideally a larger number is better, as this means that for the same output of water the quantity of desiccant needed will be lower. This, taken by itself, implies a lower system cost - both from the cost of desiccant needed, as well as the system (chamber size, insulation, 
weight). Fig 7 shows the capacity of the current desiccants used in $\mathrm{HDH}$ and AWG systems. The typical desert humidity is $25 \%$. At this level, Lithium Chloride has a peak capacity of $35 \%(w / w)$, Type 1 Silica Gel (the 'standard' type) is about $15 \%$, and Molecular Seive of about $20 \%$.

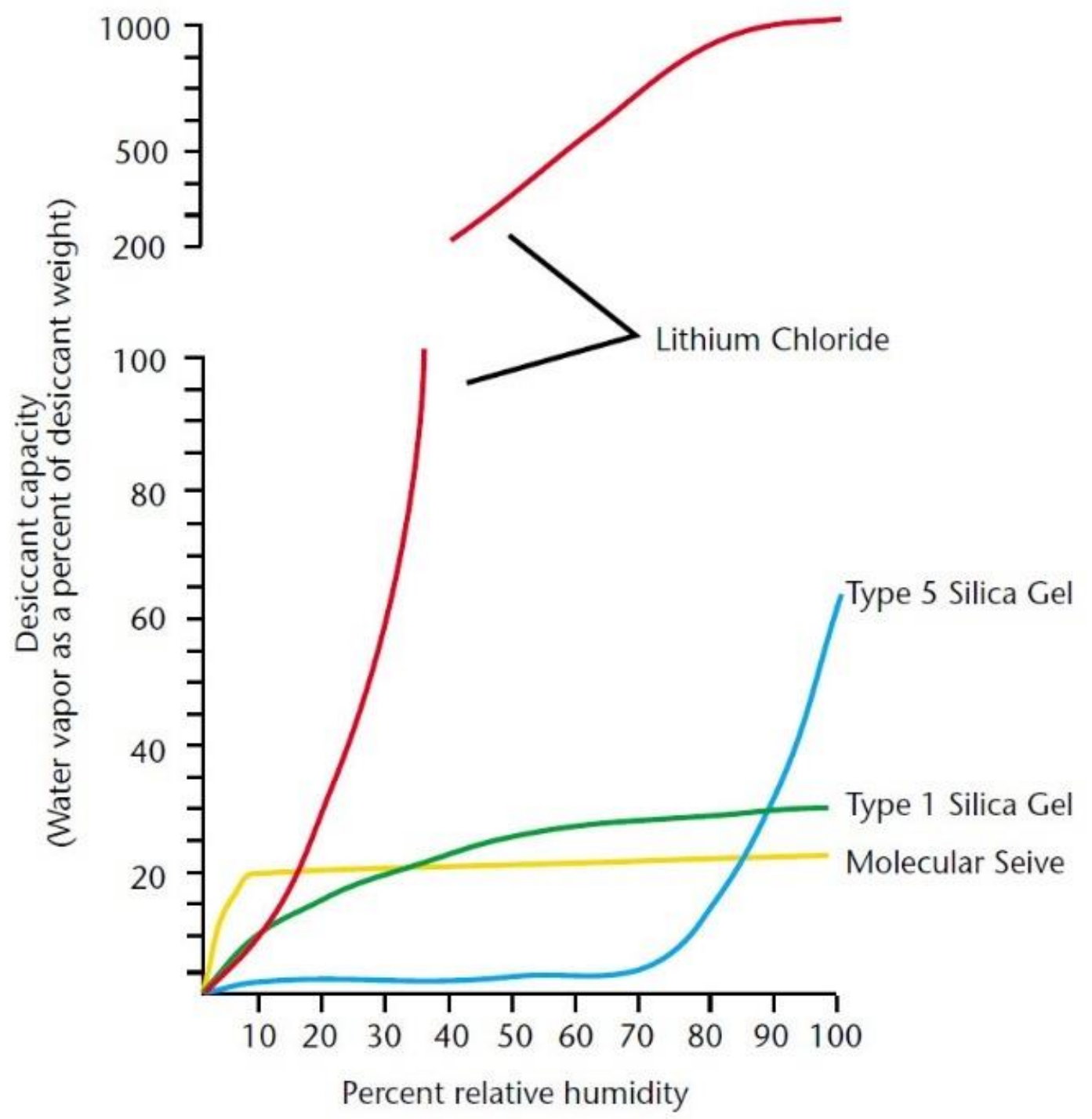

Figure 7: Desiccant Capacity [55]. Different desiccants have markedly different capacities at different temperatures. 
- Desorption temperature: a lower desorption temperature is desirable. For one, thermal losses are lower in low temperature systems, due to an exponential reduction in radiative losses. Second, at lower temperatures, solar thermal becomes practical. While solar thermal can be used to obtain extremely high temperatures, typical of concentrated solar designs [56] - these are large complex installations, better suited for power generation. Typical flat panel liquid based or direct air heating have acceptable operating efficiencies only when the temperature rise from ambient is less than $50^{\circ} \mathrm{C}$ [57].

- Toxicity and corrosion: for potable water, we need a desiccant that is not toxic, and to manage an actual plant, a non-corrosive desiccant [58] is necessary to be cost effective - from both a capital expense (capex) and operating expense (opex) perspective.

- Kinetics: The speed of adsorption and desorption have a large impact on system design. Fast desiccants mean more cycles per unit time. This can mean lower desiccant costs as well as system costs per volume of water output.

- Life: All desiccants have a finite life. It is desirable to have those that have a life in tens of years, when operated continuously.

- Form: The physical form of the desiccant in the overall design impacts total cost of ownership (TCO). For example, desiccant wheels that use coatings on fins [59], can be extremely expensive to replace periodically. Packed bed systems may offer better economics. 
- Adsorption Isotherm: The shape of the adsorption isotherm [60] will dictate its suitability in areas with lower $\mathrm{RH}$. Since water shortage is a bigger problem in dry and hot climates, a desiccant with a Langmuir type of isotherm, see Fig 8, is preferred [61]. Let us again refer to Fig 7. We see that Molecular Sieve has a sharp capacity increase up to $8 \% \mathrm{RH}$, and a flat curve from there to $100 \% \mathrm{RH}$. This is a Langmuir type adsorption isotherm, and indicates that molecular sieves are effective desiccants without any change in capacity from an ultra low humidity level of $8 \%$ up.

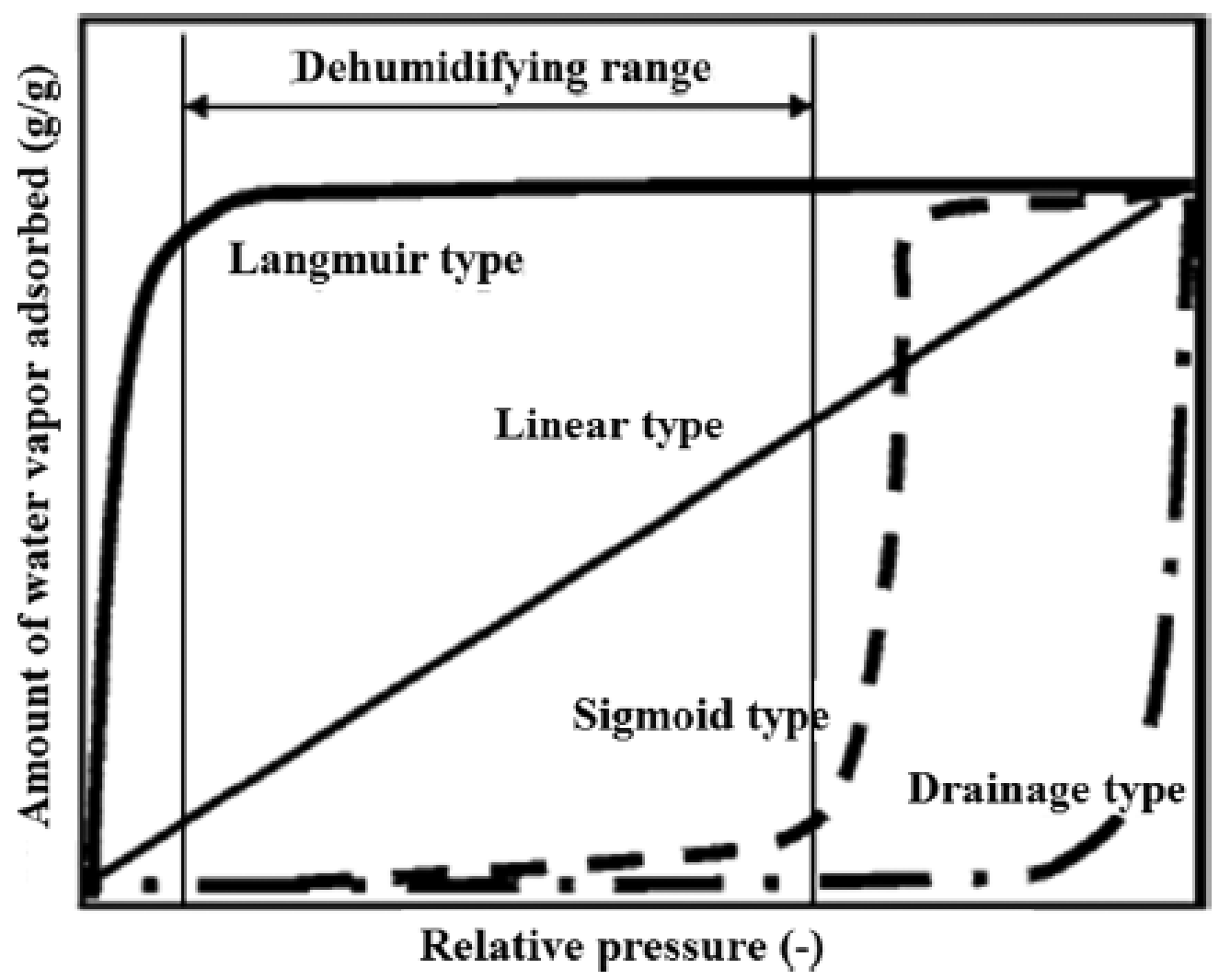

Figure 8: Water Vapor Adsorption Isotherm [62]. The Langmuir isotherm is ideal for our requirements as a desiccant with this behavior shows a uniform capacity after a minimum humidity level. 
- Impact of regeneration time on adsorption capacity: Desorb time and temperature have a significant impact on the adsorption capacity [62].

Desiccants need a finite time for recovery, and this time may be very large - with a consequent impact on thermal input and energy/exergy loss. This parameter has not been observed in the literature on thermodynamic analysis of HDH systems, even though it is significant.

- Cost: The lower the Total Cost of Ownership (TCO), the better.

A detailed analysis [63] of available desiccants is listed in Table 1. We see that zeolites need $250-350^{\circ} \mathrm{C}$ for desorption (called the 'Regeneration temperature' in the table), several others have low desorption temperatures (as low as $70^{\circ} \mathrm{C}$ ), most need relative humidity as high as $60 \%$, and some work even at $4 \% \mathrm{RH}$. 


\begin{tabular}{|c|c|c|c|c|}
\hline Adsorbent & $\begin{array}{c}\text { Adsorbent } \\
\text { temperature, } \mathrm{RH}\end{array}$ & $\begin{array}{l}\text { Adsorption capacity } \\
\text { g- } \mathrm{H}_{2} \mathrm{O} / \mathrm{g} \text {-sorb }\end{array}$ & $\begin{array}{l}\text { Textural } \\
\text { properties }\end{array}$ & $\begin{array}{l}\text { Regeneration } \\
\text { temperature }\left({ }^{\circ} \mathrm{C}\right)\end{array}$ \\
\hline \multicolumn{4}{|l|}{ Silica } & $80-150$ \\
\hline Silica gel ${ }^{[36]}$ & $27^{\circ} \mathrm{C}, 60 \%$ & $0.08-0.36$ & $2-10 \mathrm{~nm}$ & 70 \\
\hline Silica gel ${ }^{[51]}$ & $\begin{array}{c}27^{\circ} \mathrm{C}, 7 \% \\
30-40^{\circ} \mathrm{C}, 60 \%\end{array}$ & $\begin{array}{c}0.02-0.05 \\
0.34\end{array}$ & $\begin{array}{c}1 \mathrm{~nm} \\
349-690 \mathrm{~m}^{2} / \mathrm{g} \\
2-10 \mathrm{~nm}\end{array}$ & $\begin{array}{c}70 \\
70-80\end{array}$ \\
\hline $\mathrm{MCM}-41^{[36]}$ & $\begin{array}{l}27^{\circ} \mathrm{C}, 60^{\circ} \% \\
27^{\circ} \mathrm{C}, 7 \%\end{array}$ & $\begin{array}{l}0.46 \\
0.07\end{array}$ & $3.5 \mathrm{~nm}$ & 70 \\
\hline \multicolumn{4}{|l|}{ Zeolite } & $250-350$ \\
\hline Zeolite $\mathrm{Y}(\mathrm{Si} / \mathrm{Al}=5.6-220)^{[36]}$ & $\begin{array}{c}27^{\circ} \mathrm{C}, 60 \% \\
27^{\circ} \mathrm{C}, 7 \%\end{array}$ & $\begin{array}{c}0.02-0.30 \\
0-0.25\end{array}$ & - & \\
\hline $\mathrm{NaX}{ }^{[79]}$ & $30-40^{\circ} \mathrm{C}, 60 \%$ & 0.34 & $605 \mathrm{~m}^{2} / \mathrm{g}$ & - \\
\hline $\mathrm{NaY} \mathrm{Y}^{[72]}$ & $25^{\circ} \mathrm{C}, 80 \%$ & 0.25 & - & - \\
\hline Zeolite $\mathrm{A}^{[72]}$ & $25^{\circ} \mathrm{C}, 80 \%$ & 0.25 & - & - \\
\hline YZSM-20 ${ }^{[72]}$ & $25^{\circ} \mathrm{C}, 80 \%$ & 0.28 & - & - \\
\hline \multicolumn{5}{|l|}{ Activated carbon } \\
\hline AC Y $-60^{[36]}$ & $\begin{array}{l}27^{\circ} \mathrm{C}, 60^{\%} \\
27^{\circ} \mathrm{C}, 7 \%\end{array}$ & $\begin{array}{c}0.18-0.29 \\
0-0.4\end{array}$ & $2 \mathrm{~nm}$ & 70 \\
\hline $\mathrm{ACs}^{[61]}$ & $25^{\circ} \mathrm{C}, 60 \%$ & $0.25-0.50$ & $1,000-1,700 \mathrm{~m}^{2} / \mathrm{g}$ & - \\
\hline \multicolumn{5}{|l|}{ Composites } \\
\hline Silica gel/AC/ $\mathrm{CaCl}_{2}{ }^{[65]}$ & $27^{\circ} \mathrm{C}, 9^{\%} \%$ & 0.23 & $\begin{array}{l}1,117 \mathrm{~m}^{2} / \mathrm{g} \\
0.53 \mathrm{~cm}^{3} / \mathrm{g}\end{array}$ & - \\
\hline Silica gel $/ \mathrm{AC}^{[35]}$ & $27^{\circ} \mathrm{C}, 60 \%$ & 0.25 & - & - \\
\hline $\mathrm{Al}_{2} \mathrm{O}_{3}-\mathrm{SiO}_{2}^{[89,90]}$ & $23^{\circ} \mathrm{C}, 50 \%$ & 0.19 & $0.26-0.48 \mathrm{~cm}^{3} / \mathrm{g}$ & - \\
\hline $\begin{array}{l}\mathrm{CaCl}_{2} / \text { silica gel } \\
\text { SWS-1 L }\end{array}$ & $35^{\circ} \mathrm{C}, 4 \%$ & 0.19 & - & $60-80$ \\
\hline $\mathrm{SiO}_{2} \cdot \mathrm{XH}_{2} \mathrm{O} \cdot \mathrm{YCaCl}_{2}{ }^{[91]}$ & $25^{\circ} \mathrm{C}, 50 \%$ & 0.40 & & \\
\hline $\mathrm{CaCl}_{2} / \mathrm{MCM}-41^{[64]}$ & $25^{\circ} \mathrm{C}, 70 \%$ & 0.75 & $2-10 \mathrm{~nm}$ & $70-120$ \\
\hline \multirow[t]{2}{*}{$\begin{array}{l}\text { Aluminum pillared clay } \\
\text { PILC-BEN-B }\end{array}$} & $25^{\circ} \mathrm{C}, 80 \%$ & 0.21 & $350 \mathrm{~m}^{2} / \mathrm{g}$ & - \\
\hline & & & $\begin{array}{c}0.118 \mathrm{~cm}^{3} / \mathrm{g} \\
1.73 \mathrm{~nm}\end{array}$ & \\
\hline $\begin{array}{l}\text { Silicoaluminophosphate } \\
\text { SAPO-34 }\end{array}$ & $30-40^{\circ} \mathrm{C}, 60 \%$ & 0.34 & $720 \mathrm{~m}^{2} / \mathrm{g}$ & - \\
\hline \multicolumn{5}{|l|}{ Metal organic framework } \\
\hline MOF-MIL-101 (Cr) ${ }^{[79]}$ & $30-40^{\circ} \mathrm{C}, 60 \%$ & $1.5-1.7$ & $4,150 \mathrm{~m}^{2} / \mathrm{g}$ & $70-80$ \\
\hline MOF-MIL-100 (Fe) ${ }^{[79]}$ & $30-40^{\circ} \mathrm{C}, 60 \%$ & $>0.84$ & $2,300 \mathrm{~m}^{2} / \mathrm{g}$ & $70-80$ \\
\hline MOF-MIL-100 (Cr) ${ }^{[79]}$ & $30-40^{\circ} \mathrm{C}, 60 \%$ & $\sim 0.84$ & $1,980 \mathrm{~m}^{2} / \mathrm{g}$ & $70-80$ \\
\hline MOF-MIL-100 (Al) ${ }^{[79]}$ & $30-40^{\circ} \mathrm{C}, 60 \%$ & $\sim 0.84$ & $1,970 \mathrm{~m}^{2} / \mathrm{g}$ & $70-80$ \\
\hline \multicolumn{5}{|l|}{ Others } \\
\hline Dry coconut coir ${ }^{[75]}$ & $32^{\circ} \mathrm{C}, 75 \%$ & 0.30 & - & - \\
\hline Dry durian peel ${ }^{[75]}$ & $32^{\circ} \mathrm{C}, 75 \%$ & 0.17 & - & - \\
\hline $\begin{array}{l}\text { Starch-based polysaccharide } \\
\text { adsorbent }{ }^{[74]}\end{array}$ & $25^{\circ} \mathrm{C}$ & Dew point of $-63^{\circ} \mathrm{C}$ & - & - \\
\hline
\end{tabular}

Table 1: Adsorption parameters of some adsorbents [63]

The "Adsorbent temperature, $\mathrm{RH}$ " is the temperature and $\mathrm{RH}$ the desiccant was subject to for adsorption, while the "Regeneration temperature" is the desorption temperature. Looking at this table alone, it appears that some of these are suitable - the desorption temperatures suit our requirements of low temperature desorption, and the capacity is $\sim 40 \% \mathrm{w} / \mathrm{w}$. However, none of the desiccants listed in the table meet all of our requirements, especially when we 
are considering kinetics (not listed in the table) which can be several hours for adsorb and desorb processes.

We now review the available literature on the thermodynamics of $\mathrm{HDH}$ systems. The basic surface vapor pressure-humidity relation is shown in Fig 9 the surface vapor pressure is inversely proportional to the desiccant's water adsorption capacity. It is observed that there is a temperature increase with sorption (humidification), and the reverse with desorption (dehumidification).

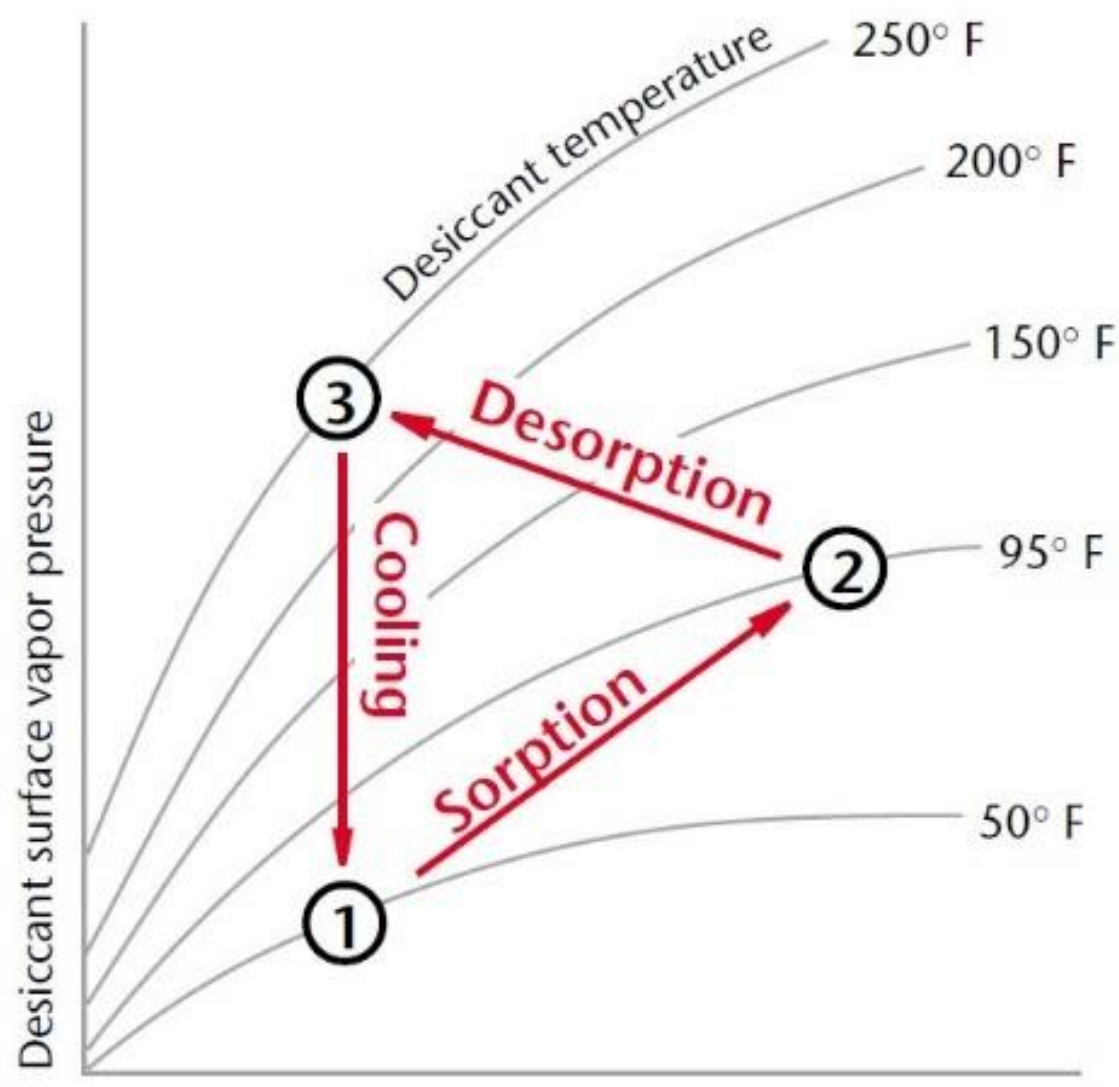

Desiccant moisture content

Figure 9: Vapor Pressure vs Moisture [55]. The stages of Adsorption, Desorption and Cooling of a desiccant, along with its moisture content. 
A potential problem can be identified when we consider low temperature desorption desiccants. Since adsorption will cause the desiccant temperature to increase, the water holding capacity will reduce. MoF's [64] have been shown to adsorb $120 \%$ by weight [65] - this however takes several hours. Were this adsorption to take place in a few minutes, the heat of condensation, $2230 \mathrm{~J} / \mathrm{gm}$, would substantially increase the temperature of the desiccant, pushing the desiccant to adsorb sub-optimally [66]. This will become a critical issue with desiccants that have fast kinetics. It would appear then, that desiccants with fast or ultra-fast kinetics that desorb at low temperatures, will actually perform better with low capacity, or where only a limited adsorb capacity is used.

An alternate strategy, appropriate for AWG systems (though not for $\mathrm{HDH}$ systems, like desalination), is to subject the desiccant to excess air flow (even after complete adsorption) which will cool the desiccant by carrying away the heat of condensation. This implies excess energy is required for the air pump here atmospheric air is free, and the pump is the only input power required.

The cooling stage, 3 to 1 , in Fig 9, is also managed during the initial part of the adsorb cycle in the AWG system. This reduces system complexity, as the desiccant is subject to 3-1-2 in the adsorb phase, and 2-3 in the desorb phase.

Whereas Fig 10 illustrates the temperature - moisture for a rotary wheel system, this is generally applicable to all HDH systems. Note the "Reactivation" in this figure means "Desorb", for our purposes. 'Gr' is grains, an old English unit of moisture [67]. 


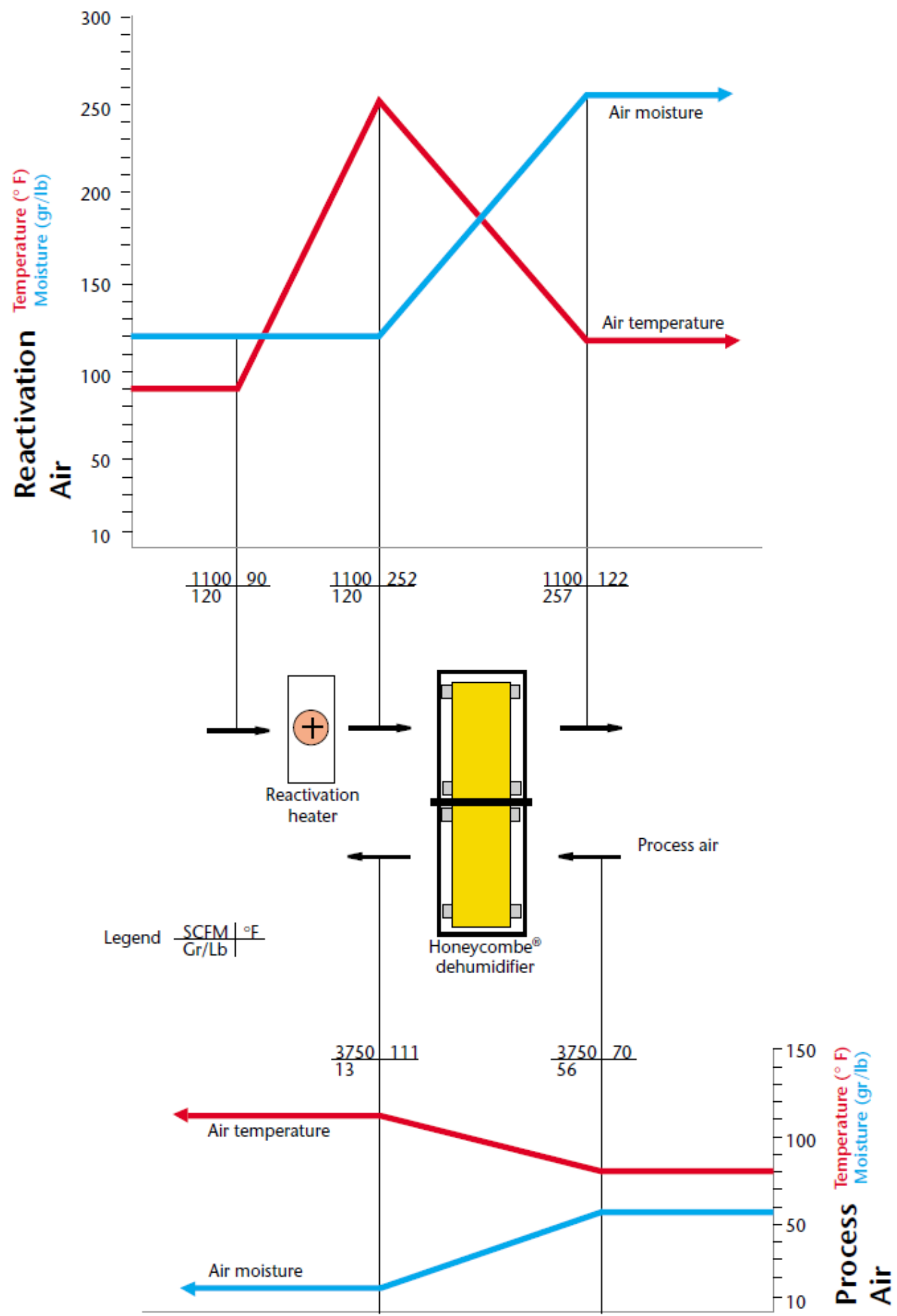

Figure 10: Airflow temperature and humidity changes [55] in a rotary $\mathrm{HDH}$ system, showing the temperature and moisture changes over the Adsorption and Desorption stages. 
Since the temperature of the dehumidification air stream falls as it picks up moisture, the desiccant is subject to a lower temperature at exit side, and two, the absolute water holding capacity of the air reduces. We saw earlier these systems operate best when the exit temperature is high enough that ambient temperature cooling will cause a reasonable quantity of water to condense from the air stream. This implies that the entry temperature to the desiccant in the desorb phase needs to be higher than its desorb temperature - equivalent to an overpotential. This overpotential can be reduced by optimizing the packed bed thickness as well as controlling the mass flow of air.

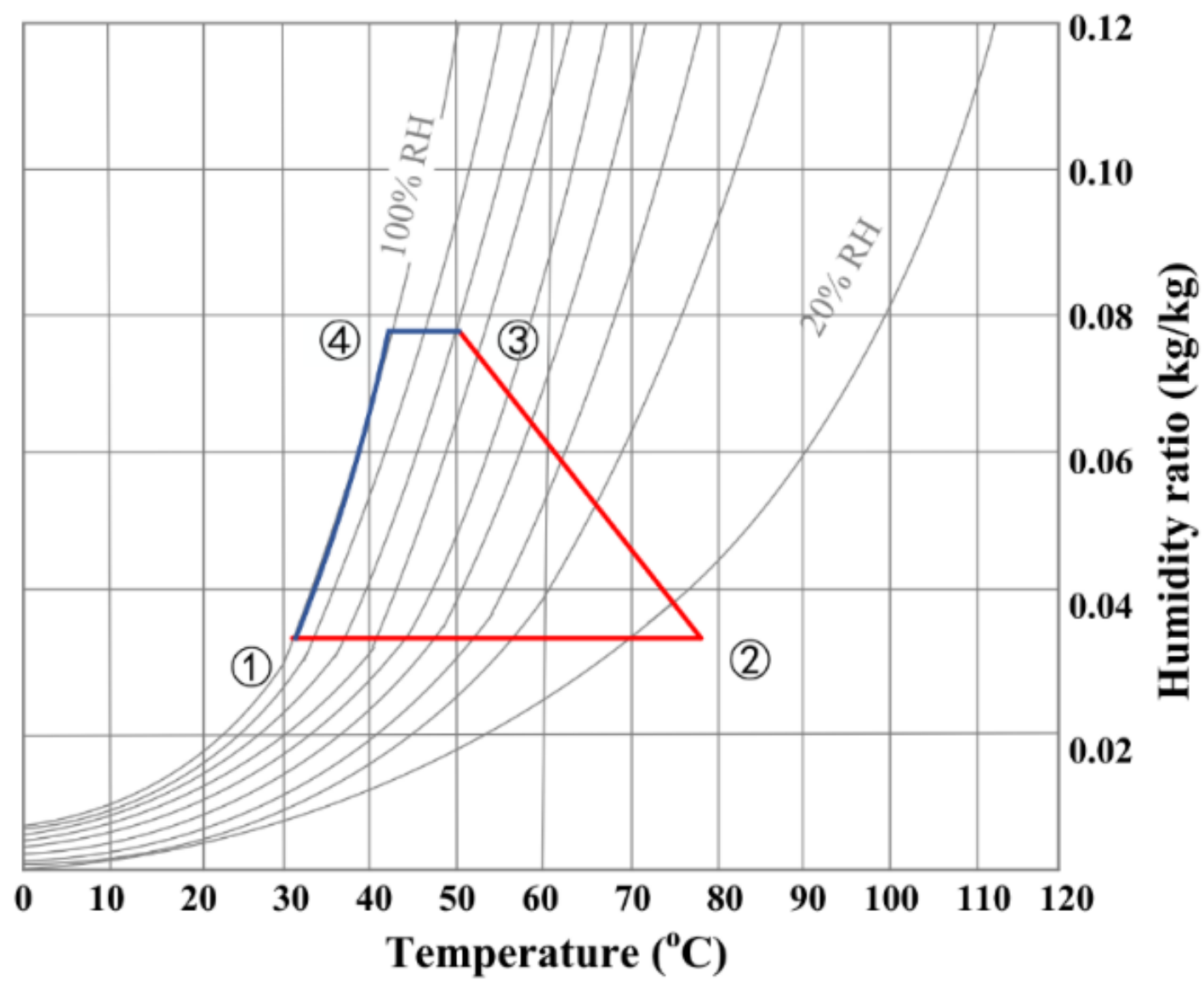

Figure 11: Psychrometric chart [36] illustrating the humidity ratio (water content) of air in the dehumidification loop. 
We now look at the temperature and absolute humidity in the dehumidification loop. In Fig 11, process 1-2 is heat addition to the air. The temperature scales are typical of simple (flat plate) solar driven heating systems. Process $2-3$ is the dehumidification process, with the typical exit humidity around $80 \% \mathrm{RH}$. Process 3-4-1 is the condensation phase. Condensation starts at 4 , the dew point.

Consider a Thermo Gravimetric Analysis - Differential Scanning Calorimetry, TGA-DSC (TGA for short in this document) of a desiccant, as shown in Fig 12. The desiccant desorption knee $\left(73^{\circ} \mathrm{C}\right.$ in Fig 12$)$ is the point 3 of Fig 11. The temperature at point 2 is necessarily higher than at point 3 since heat is required for desorption which causes the temperature to come down. The exit temperature from the desiccant would need to near the desorption knee, for complete desorption. 


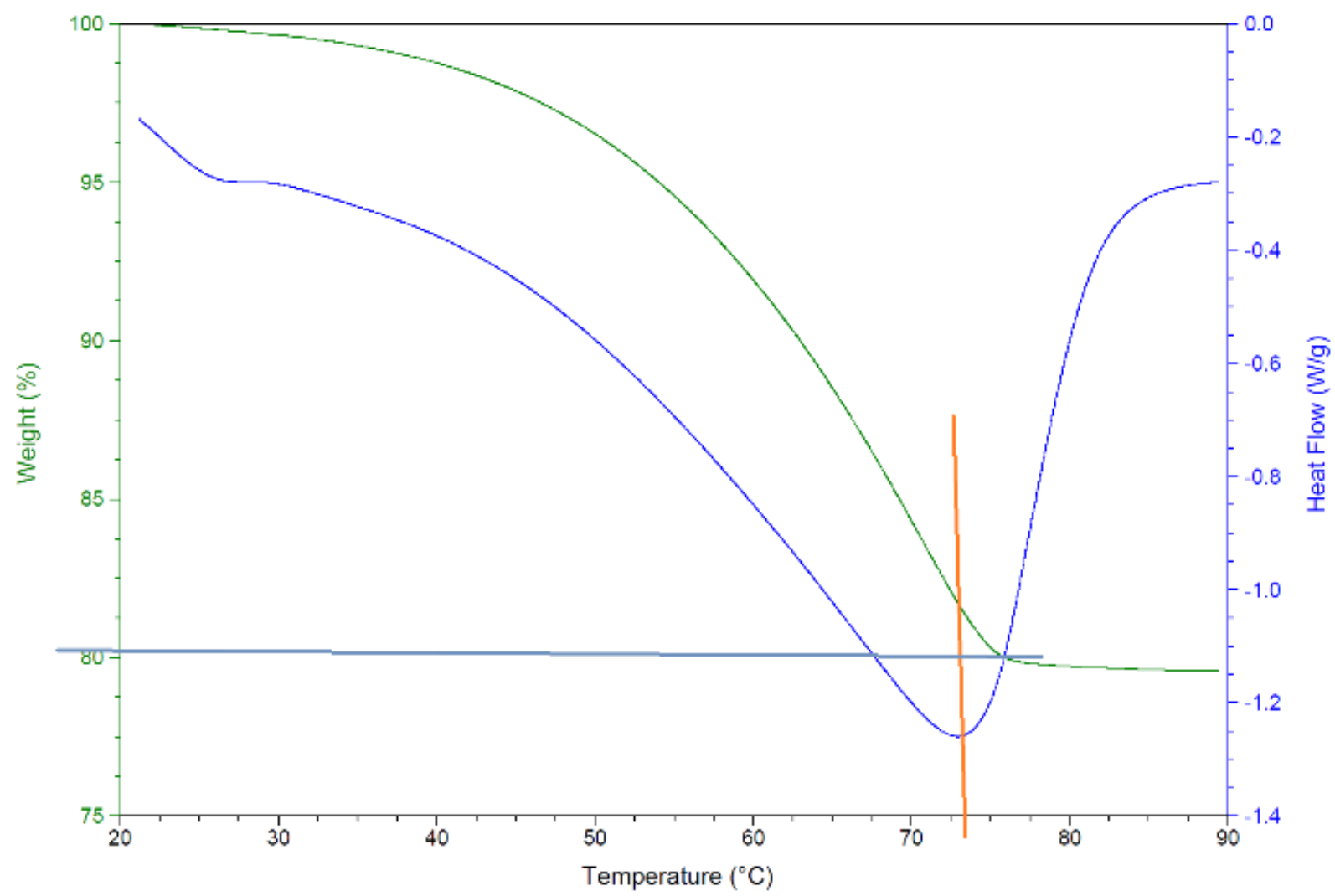

Figure 12: TGA for a LAO sample showing the point of maximum heat flow (representing the desorb temperature) and the maximum desiccant capacity.

Thermo Gravimetric Analysis is a method of thermal analysis in which the mass of a sample is measured over time as the temperature changes, while Differential Scanning Calorimetry measures the energy input or released by the sample over the same time. These simultaneous measurements provide information about physical phenomena, such as phase transitions, absorption and desorption; as well as chemical phenomena including chemisorption, thermal decomposition, and solid-gas reactions (e.g., oxidation or reduction). Figure 13 is an illustrative graph [68]. 


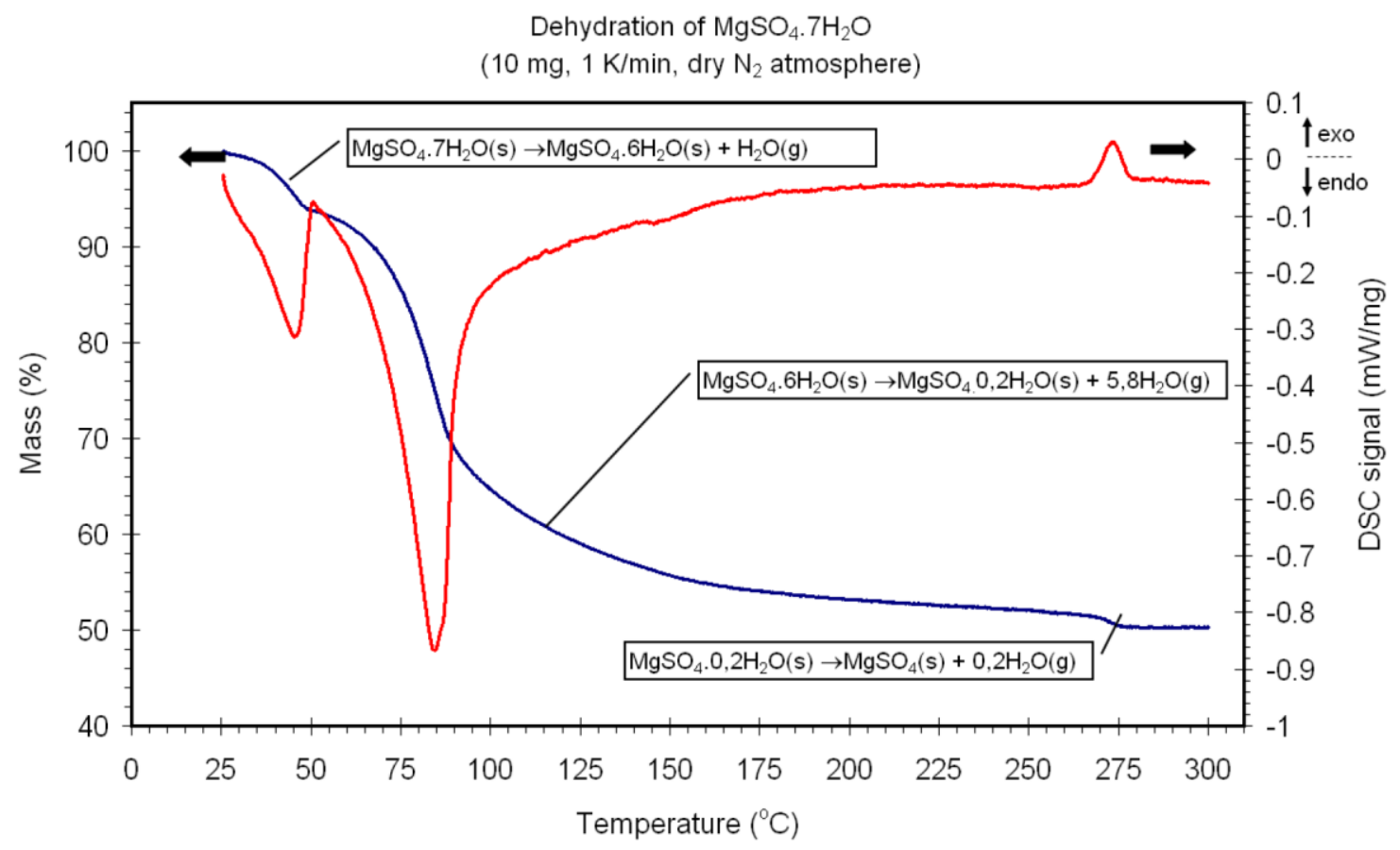

Figure 13: A typical TGA-DSC curve for dehydration of $\mathrm{MgSO}_{4} .7 \mathrm{H}_{2} \mathrm{O}$. The blue line denotes TGA curve and the red line denotes the DSC curve.

The graph indicates that dehydration of $\mathrm{MgSO}_{4} .7 \mathrm{H}_{2} \mathrm{O}$ proceeds in three discrete steps as illustrated in Figure 13: the first dehydration reaction occurs at low temperature $\left(<50^{\circ} \mathrm{C}\right)$ and involves the loss of one water molecule. The largest number of water molecules is dehydrated during the second reaction $\left(50^{\circ} \mathrm{C}\right.$ to $\left.275^{\circ} \mathrm{C}\right)$, which also allows storing the largest amount of energy of the three dehydration reactions. The last water molecules are dehydrated at a high temperature $\left(\sim 275^{\circ} \mathrm{C}\right)$. In contrast to the first two dehydration reactions, the third dehydration reaction involves an exothermic process (indicated by the positive DSC signal). 
The literature is rich in HDH systems used for desalination [18, 19, 22-25, $28,31,32,69,70]$. Complex designs have been evaluated for maximum GOR, for maximum reuse of thermal energy. Fig 14 is one such design [32], where using a stratified design different air streams at different temperatures from the humidifier are directed to sections in the humidifier for maximum thermal efficiency, which contribute to improving GOR.

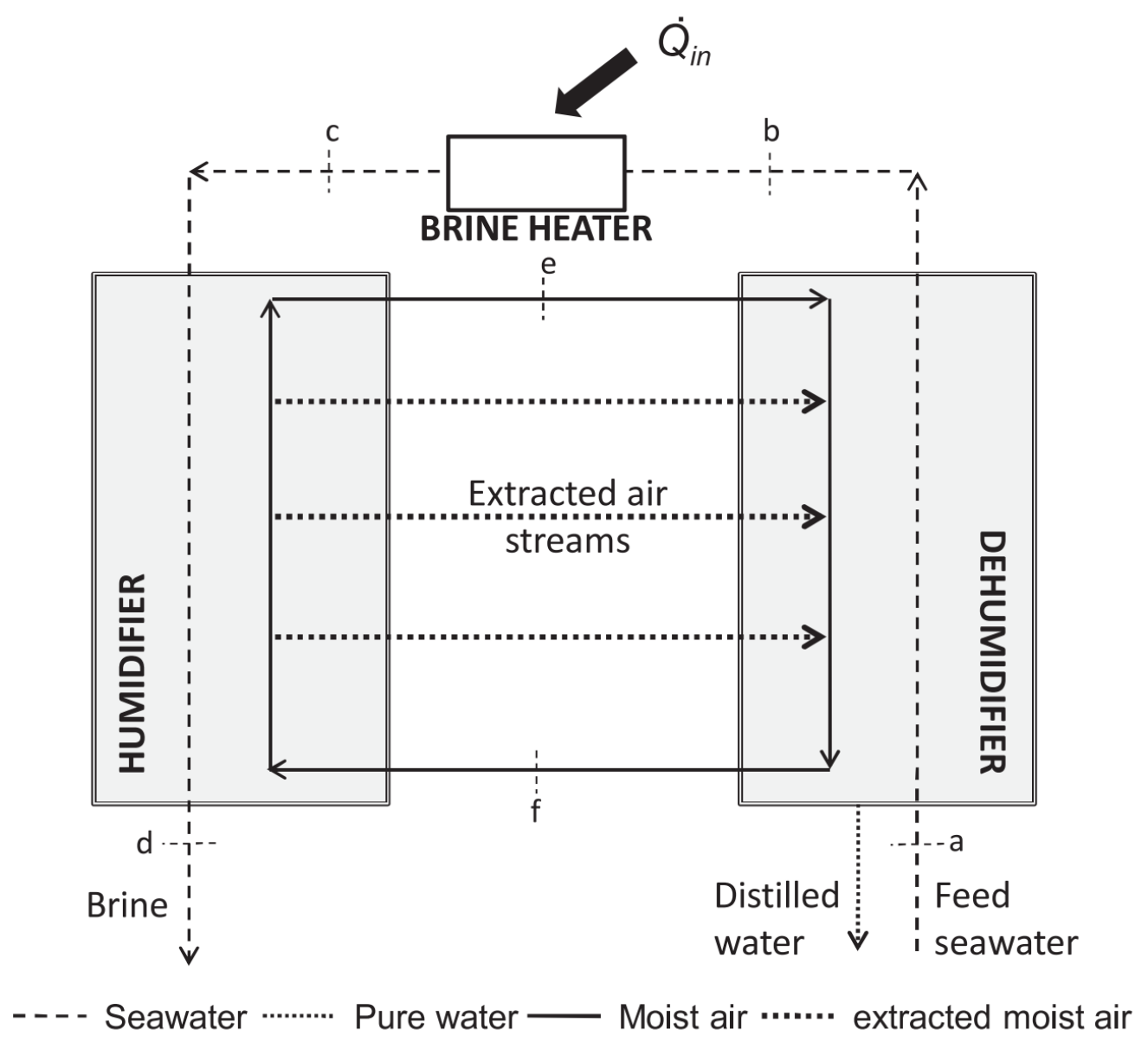

Figure 14: Desalination design for maximum GOR - showing a stratified design

The literature is however very scarce on the thermodynamics for AWG systems. Rotary desiccant based cooling devices, which can be considered to be 
closely related to AWG systems have been described along with thermodynamic calculations [71].

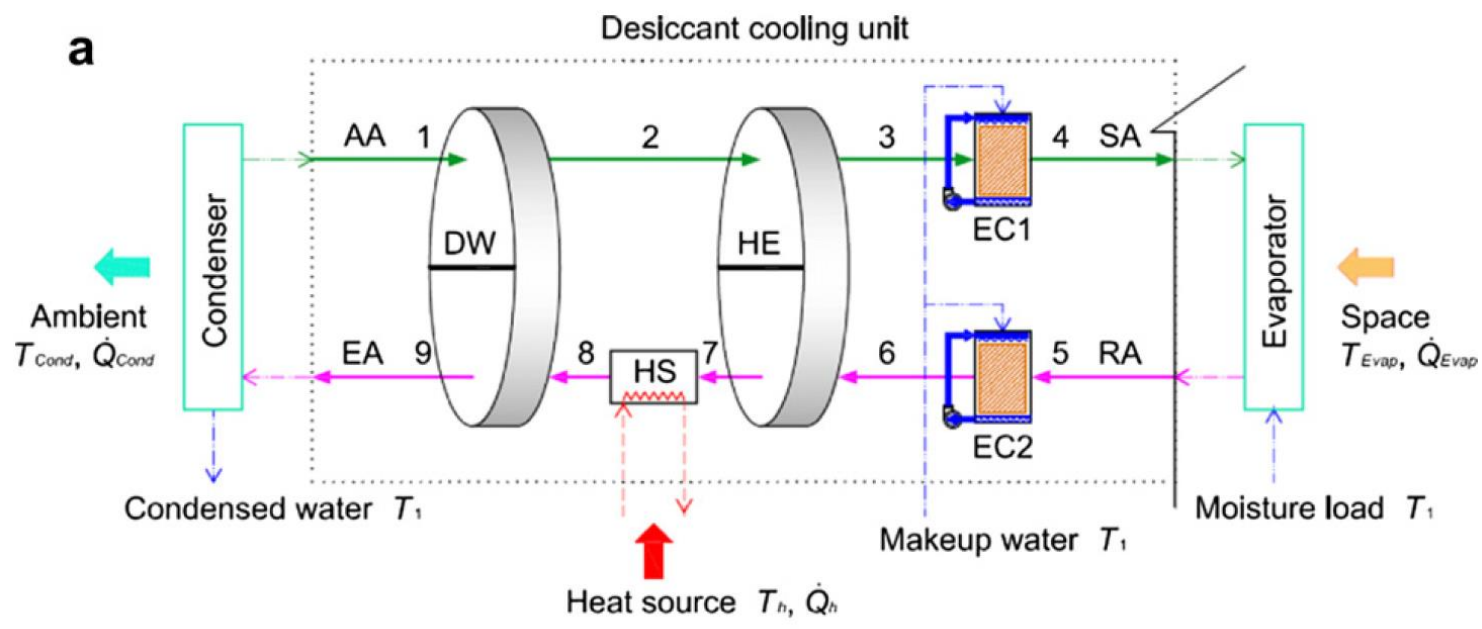

b

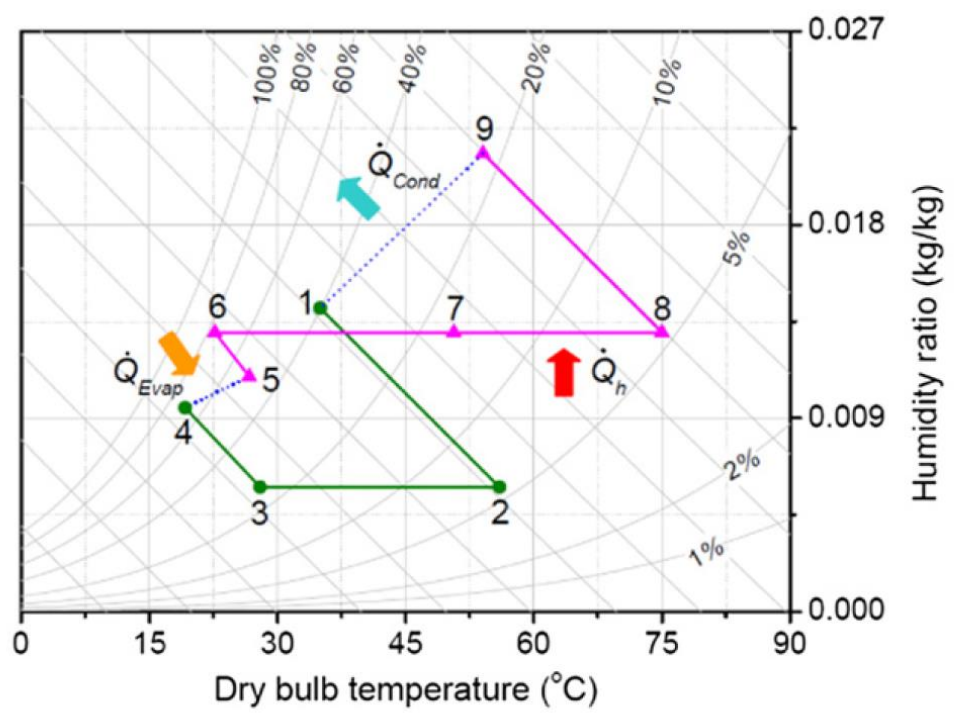

Figure 15: Desiccant Cooling System [71], using a heat recovery wheel. The colored lines in (b) correspond to the stages in (a).

We can observe from Fig 15, that there is heat recovery from the latent heat during adsorption that is used in the desorption phase. This heat recovery is not a possibility in low temperature AWG systems, as the temperature differences are insufficient for the air-to-air heat exchangers to be effective (even 
ignoring the power requirements for the pumps, which by themselves are not small). In the figure DW is the Desiccant Wheel, HE is the Heat Extraction wheel, HS is the Heat Source, EC1 \& EC2 are Evaporative Coolers, AA is Ambient Air, SA is Supply Air, RA is Return Air and EA is Exhaust Air.

Low temperature AWG systems operate as per Fig 11. The maximum theoretical GOR is one since the input energy is used in the desorb cycle to pick up moisture and lost to ambient during condensation - there is no recovery of thermal energy. From a thermodynamics viewpoint, therefore, there is little analysis of value to perform in stand-alone low-temperature desiccant based AWG systems, and this has therefore not been taken up in this study.

A GOR of one appears, at first sight, to negate any further research. However, from a systems perspective, efficiency is often not the only consideration. As an example, using conventional energy sources and $\mathrm{HDH}$ systems at high temperature, we can achieve a high GOR [23], yet if we were to consider affordability, supportability and operating costs, and the fact that solar thermal energy is practically free, GOR can be deprioritized. 
The table below summarizes the requirements for an ideal desiccant:

\begin{tabular}{|c|c|}
\hline Specification & Range / limit required \\
\hline $\begin{array}{l}\text { Adsorption } \\
\text { capacity }\end{array}$ & $\begin{array}{l}\sim 20 \% \text { w/w } \\
\text { As the kinetics we are aiming for are fast, in minutes, the } \\
\text { heat of adsorption will cause a rapid rise in the desiccant } \\
\text { temperature. We also require a low desorption } \\
\text { temperature. The specification is therefore set for a } \\
\text { relatively low adsorption capacity. }\end{array}$ \\
\hline $\begin{array}{l}\text { Desorption } \\
\text { temperature }\end{array}$ & $\begin{array}{l}>60^{\circ} \mathrm{C}<70^{\circ} \mathrm{C} \\
\text { Ambient temperatures in most parts of the tropics reach } \\
\sim 40^{\circ} \mathrm{C} \text {. The heat of adsorption will raise the desiccant } \\
\text { temperature. Too low a desorption temperature will result in } \\
\text { poor adsorption. } \\
\text { The target setup uses flat plate solar thermal air heaters. } \\
\text { Typically, these deliver no more than } 90^{\circ} \mathrm{C} \text {. Desorption } \\
\text { causes this temperature to fall. We therefore need the } \\
\text { desorption temperature to be lower than this. }\end{array}$ \\
\hline Toxicity & Negative \\
\hline Corrosive & Negative \\
\hline $\begin{array}{l}\text { Kinetics (adsorb } \\
\text { / desorb) }\end{array}$ & $\begin{array}{l}\text { Minutes and not hours } \\
\text { A simple and compact system will deliver higher water } \\
\text { output if multiple cycles can be run during the } \\
\text { approximately } 5 \text { hours of sunshine. The more the cycles we } \\
\text { can run, the more the output. Here we are assuming that } \\
\text { the area needed (the real estate) for the flat panel solar } \\
\text { thermal collectors are not the limiting factor in the system } \\
\text { design. }\end{array}$ \\
\hline Life & $\begin{array}{l}\sim 20 \text { years } \\
\text { Longer life desiccants imply lower capital and operating } \\
\text { costs. }\end{array}$ \\
\hline Form & $\begin{array}{l}\text { Solid } \\
\text { Simple, easy to maintain systems are best managed with } \\
\text { solid desiccants. }\end{array}$ \\
\hline $\begin{array}{l}\text { Absorption } \\
\text { Isotherm }\end{array}$ & $\begin{array}{l}\text { Langmuir } \\
\text { The desiccant must perform well and deliver substantially } \\
\text { the same water output irrespective of the } \mathrm{RH} \text {, as well as } \\
\text { work well in desert conditions. }\end{array}$ \\
\hline
\end{tabular}


\begin{tabular}{|l|l|}
\hline Material & Inorganic, earth abundant
\end{tabular}

Both for long life and cost factors.

Table 2: Properties of an ideal desiccant 


\section{SCOPE}

This research is focused on the synthesis, characterization and validation of a desiccant that will meet the requirements of an ideal desiccant, as detailed in Table 2.

We have seen from detailed literature reviews that none of the desiccants available either commercially or lab scale, meet all these requirements.

The scope of this research is to:

Identify potential desiccant candidates from literature reviews.

Starting with the first one, we synthesize the material in nano powder form.

Characterize the nano powder using TGA to verify that the material meets the basic requirement of adsorption capacity and temperature of desorption.

If the material meets these requirements, use SEM and XRD to confirm the material phase and 2D morphology.

To perform detailed tests on the kinetics of adsorption and desorption, to validate that the time scales we seek are achieved.

To perform tests on coating or directly synthesizing this material on metal surfaces and verify performance of such coatings.. 
To convert the nano-powder into a solid extrudate and test that the extrudate continues to meet our requirements.

To test the extrudates in a prototype to obtain real-world water output from the system.

While we do require a system (which we did design and setup to test the desiccants) (Appendix 2) the scope of this research is primarily focused on the identification, synthesis and characterization of a desiccant that will meet or exceed the specifications in Table 2. 


\section{METHODS}

A review of materials was undertaken to identify potential earth abundant inorganic solid materials that have desiccant properties, which are not commercially available and which are chemically stable.

This review indicated that Lithium Aluminate, $\mathrm{LiAlO}_{2}(\mathrm{LAO})$ has been known to be a water adsorbent, and significantly, of being stable in water. Using samples of surface area (as low as $0.2 \mathrm{~m}^{2} / \mathrm{gm}$ ) it was shown [72] that the adsorption was:

$$
\begin{aligned}
& Q_{a d}=2.6 \times 10^{7} A P^{1 / 2} \exp \frac{32000}{R T} \\
& \text { where } \left.Q_{a d} \text { : Amount of adsorbed water ( } \mathrm{mol}-\mathrm{H}_{2} \mathrm{O} / \mathrm{mol}-\mathrm{LiAlO}_{2}\right) \\
& \text { A: BET surface area of } \mathrm{LiAlO}_{2}\left(\mathrm{~m}^{2} / \mathrm{g}\right)
\end{aligned}
$$

We therefore considered the use of Lithium Aluminate as a promising desiccant, and from Eq. 1, we could safely assume that nano material would enable significant adsorption in terms of grams of water/grams of LAO. Using traditional sol-gel methods, the literature demonstrated [73] that surface areas of about $70 \mathrm{~m}^{2} / \mathrm{gm}$ was achievable. 
A UofL patented process (SolvoPlasma ${ }^{\mathrm{TM}}$ ) [74] was used to attempt synthesis of potential desiccant material in the nano powder form. The ratio of precursors, method of mixing and solvents are to be varied to arrive at a sample of a nano material.

If successful in the synthesis of the nano material, TGA was performed to validate the desorb temperature and desorb capacity. This method of analysis also provides insights into the desorb process (physisorption or chemisorption) and desorption energy required.

\section{Precursor preparation}

Different molar ratios of Aluminum Oxide (Nanoarc, Alfa Aesar) : Lithium hydroxide, anhydrous powder, 99.995\% metals basis, (Alfa Aesar) was weighed. The mixture was well mixed by hand in a mortar for about 3 minutes to obtain a homogeneous solid mixture. Deionized (DI) water was slowly added while the mixture was continuously hand ground to form a fine paste. For a total mixture of about $50 \mathrm{~g}$, the time required was 10 minutes.

\section{Plasma synthesis}

The Atmospheric Upstream Microwave Plasma Reactor in the Plasma Research Lab, was used for synthesis of nano-LAO.

The procedure to operate the plasma for our synthesis was:

- Flip power strip and back switches

- Turn on water (knob turns counter clockwise)

- Turn on Air to $10 \mathrm{~L} / \mathrm{min}$ (counter clockwise)

- Turn on Argon to $1 \mathrm{~L} / \mathrm{min}$ (counter clockwise) 
- Set the black knob to 1200

- Put the copper stick in and rotate around while flipping the switch to on to spark the

- flame

- Turn black knob down to 800 slowly

- Turn off the Argon

A thin coating of the mix, about $1 \mathrm{~mm}$ was applied on half of one side of a 1" $x$ 3", 3mm quartz slide. This was exposed for 60 seconds to the microwave plasma - mix facing down over the plasma flame in a gentle 'painting' motion. The slide was cooled by placing on a thick sheet of aluminum and the powder scraped off with a blade. The slide was then reused as is.

\section{$\underline{\text { Nano-LAO tests - Basic }}$}

The synthesized material was tested using the following techniques to assure us that we had obtained Nano-LAO, without using time consuming testing with SEM and BET.

1. A part of the synthesized powder was measured. This was then stirred in DI water. The mixture was filtered with a filter paper. The distillate was slowly heated till dry. The residue was then weighed. X-ray diffraction (XRD) of this residue was conducted and expected to show essentially $\mathrm{LiOH}$. The molar ratio of the mixture gives the theoretical weight of unreacted $\mathrm{LiOH}$ to be expected, and from the actual weight of unreacted $\mathrm{LiOH}$ from the distillate, we could conclude the reaction completion status in the formation of LAO. 
2. Another part of the synthesized powder is lightly dry ground by hand in a mortar. The powder is left open in a petri dish for $30+$ minutes in the lab as- the ambient environment of the lab is sufficient. This powder is then subject to TGA.

Once we satisfy ourselves that the material synthesized was probably nanoLAO, we proceed with detailed characterization:

1. XRD to confirm that the material phase is $\mathrm{LiAlO}_{2}$

2. SEM to confirm 2D morphology

If the results meet specifications, adsorb and desorb kinetic tests were performed. This was accomplished by forming thin coatings of the LAO nano material, using a variety of carriers, and subjecting these to air at different temperatures and humidity to determine adsorption kinetics. Once adsorption is complete, the coatings are to be heated in an oven at the desorption temperature for different durations, to determine the desorption kinetics of the material.

If the kinetics were suitable, material was prepared in bulk (about 100+ grams). This was converted to aggregate particles (2-5 mm in diameter), by mixing the nano powder with a binder (like Ethyl Cellulose) and liquid carrier (like Methanol), extruding the paste, drying and sintering the extrudates to a temperature that causes the binder to burn away, to result in a highly porous and 
sintered extrudate. These were then broken in a pestle to smaller $(2-5 \mathrm{~mm})$ aggregates

These aggregates were subjected to TGA and then to kinetics testing, to validate that the characteristics have not changed in the process of conversion from nano powder to aggregates

These aggregates were to be finally placed into the system prototype (described in Appendix 2) to arrive at actual capacity of water extracted per hour per kg of desiccant, under ambient conditions. This step was not done by us, and it is expected to be completed as part of further research. 


\section{RESULTS \& DISCUSSION}

This chapter looks at the results obtained and discusses how these fit, or do not fit the hypothesis that we have obtained a suitable desiccant.

Basic validation performance of the Lithium Aluminate materials of the literature [72] was performed first (don't understand this, are you saying you have literature data to compare with?). Samples of Lithium Aluminate were prepared using standard sol-gel techniques. Fig 16 is the Scanning Electron Microscope (SEM) image of the resulting powder. We see rod-like structures, about $1 \mathrm{~nm}$ in thickness and 1 to $8 \mathrm{~nm}$ in length, indicating that we have obtained a nano powder.

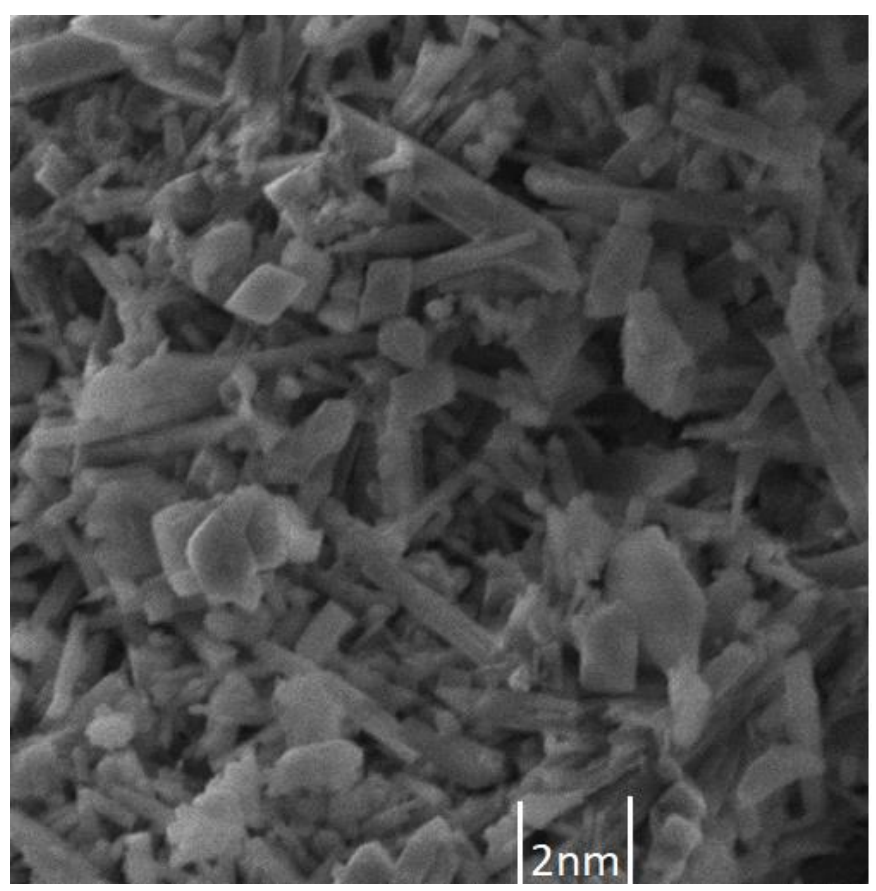


Figure 16: SEM of sol-gel synthesized LAO showing nano rods 
This powder was then subject to TGA. For this, and all future TGA tests, the system was setup as follows:

1. The TGA crucible is cleaned of impurities by heating to red hot on a Bunsen burner, till the flame color is blue. The crucible is cooled.

2. Initial temperature - ambient. It is important that in case the TGA had been in use, that it is given adequate time to cool down to ambient temperature before placing the crucible.

3. The empty crucible is placed in the TGA and tare set to zero.

4. The crucible is removed and the material sample loaded.

5. The gas source is set to 'Air'.

6. The ramp up rate is set to $2^{\circ} \mathrm{C} /$ minute.

7. The maximum temperature is set to $100^{\circ} \mathrm{C}$.

8. On reaching the maximum temperature, the ramp down rate was set to "cool as fast as possible", as we are not testing the TGA plot in the cooling cycle.

9. The sample is removed after the cooling fan shuts down. 
Fig 17 is the TGA of this sample It was observed that desorption reached its maximum at about $90^{\circ} \mathrm{C}$. This indicated that LAO was a promising lowtemperature desiccant. The moisture desorption was $5 \% \mathrm{w} / \mathrm{w}$. We therefore decided to proceed to the next step in the experimental flow - that of synthesizing LAO in nano material form.

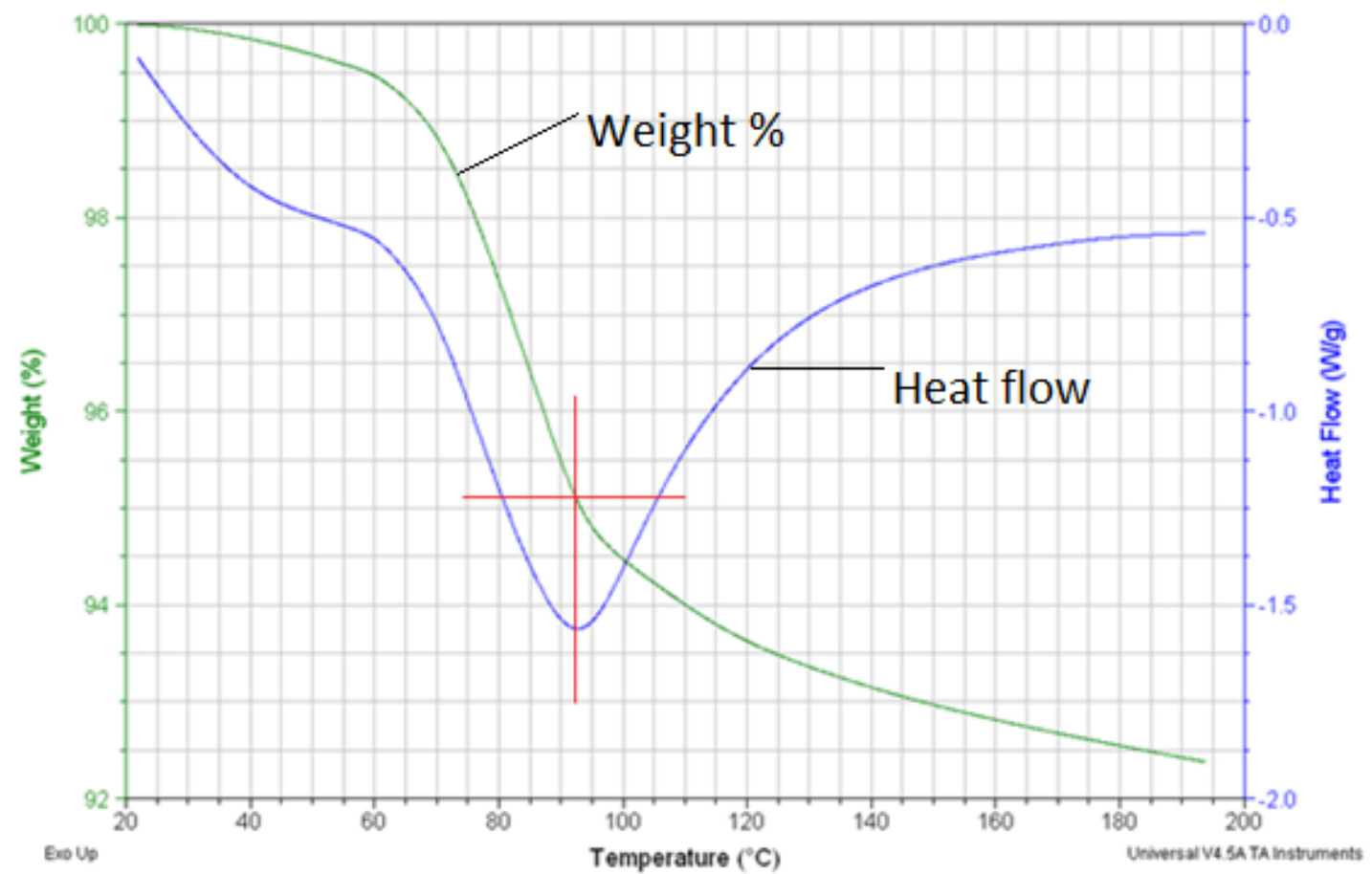

Figure 17: TGA of sol-gel LAO that shows a marked inverse peak in the heat flow

It is also important to appreciate the basic mechanism involved with moisture adsorption by LAO. Based on previous work, it was shown that the process was dissociative chemisorption [75]. The mechanism [72] is theorized to involve the reaction of moisture with $\mathrm{Li}$ atoms on the surface of the sorbent [76] creating a coating under a pure chemisorption step, while desorption is a reverse dissociative step. The exact reaction was not identified in this investigation. From 
the literature, it has been shown that $\mathrm{LiAlO}_{2}+4 \mathrm{H}_{2} \mathrm{O} \rightarrow \mathrm{Li}\left[\mathrm{Al}\left(\mathrm{H}_{2} \mathrm{O}\right)_{2}(\mathrm{OH})_{4}\right]$ a possibility - this reaction occurs in the presence of $\mathrm{LiOH}$. The activation energy of the actual reaction (chemisorption) has been estimated [72] to be 32.2 $\mathrm{kJ} / \mathrm{mol} . \mathrm{k}$

We can therefore theorize that this is not a physical adsorption (physisorption). Instead it appears that this is a chemisorption involving the diffusion of atoms in the solid phase. These atoms are likely to be lithium (which is provided by Lithium Hydroxide). Lithium Hydroxide must therefore be available, and that a pure LAO substance would not perform without an additional source of Lithium.

LAO nano material was prepared using the SolvoPlasma ${ }^{\text {TM }}$ process with 1:3, 1:5, 1:6 and 1:7 molar ratio of $\mathrm{AL}_{2} \mathrm{O}_{3}: \mathrm{LiOH}$, as described in the Methods chapter.

Material preparation had a significant impact on the synthesis. Different approaches to mix the precursors were tried - including sonication, ball mills etc. What worked best for us was to dry mix the materials together, add water and mechanically grind the paste by hand in a pestle. In the other cases the synthesized LAO did not result in the white flaky (similar to corn flakes) on synthesis that we had come to know was a nano LAO material, by simple visual inspection. 
The synthesized material was tested as described in the Methods chapter for assurance that the plasma process was executed correctly and that nanoLAO was formed.

It was observed from the experiments above that reaction completion was accomplished only at and above 1:6 molar ratio. Based on the actual stoichiometry, and synthesis, we had therefore established that excess $\mathrm{LiOH}$ was required to obtain nano Lithium Aluminate.

At the same time, the literature showed that an additional source of lithium was also needed for water adsorption. To validate this a TGA analysis was conducted:

Fig $18,19,20 \& 21$ are the TGA analysis of freshly synthesized LAO (performed at a temperature ramp rate of $2^{\circ} \mathrm{C} / \mathrm{min}$ ). Fig 21 , the TGA for $1: 3$ molar mix, clearly lacks the "knee" in the heat flow rate - indicating that there is no zone of desorption. Fig 19,20 \& 21 all have the "knee", as well as $12-22 \%$ reduction in the weight of the desiccant, implying a clear zone of desorption. Table 3 is a summary of the findings. The "knee" temperature is the inflection point on the heat flow plot.

\begin{tabular}{|c|c|c|}
\hline Molar Ratio of $\mathbf{m i x}$ & Desorption w/w \% & "Knee" temp. C \\
\hline $1: 3$ & 3 & $?$ \\
\hline $1: 5$ & 14 & 58 \\
\hline $1: 6$ & 16 & 68 \\
\hline $1: 7$ & 21 & 72 \\
\hline
\end{tabular}

Table 3: TGA summary of different pre-cursor molar mixes 


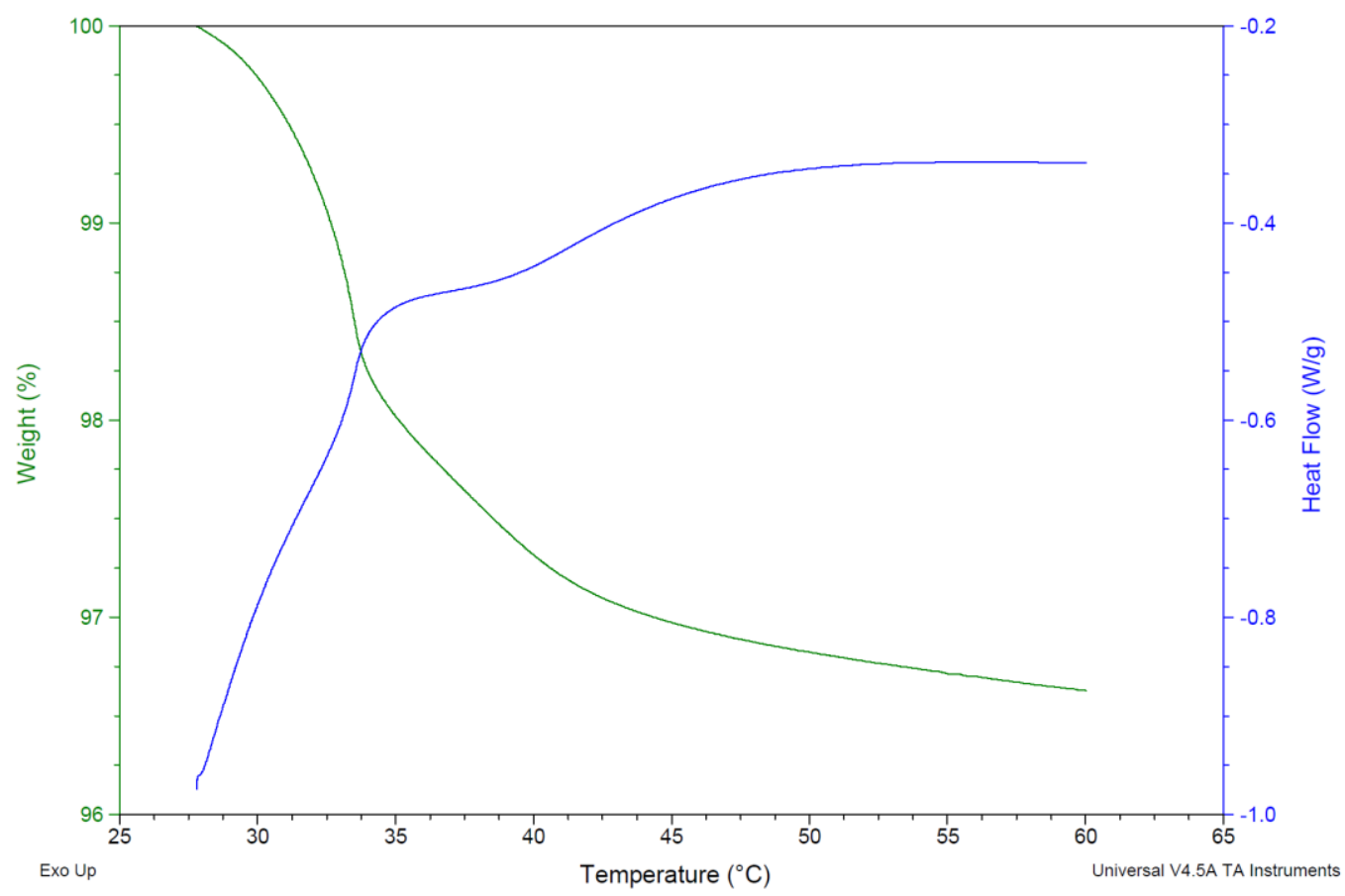

Figure 18: TGA of LAO from 1:3 molar pre-cursor mix that does not have a characteristic peak

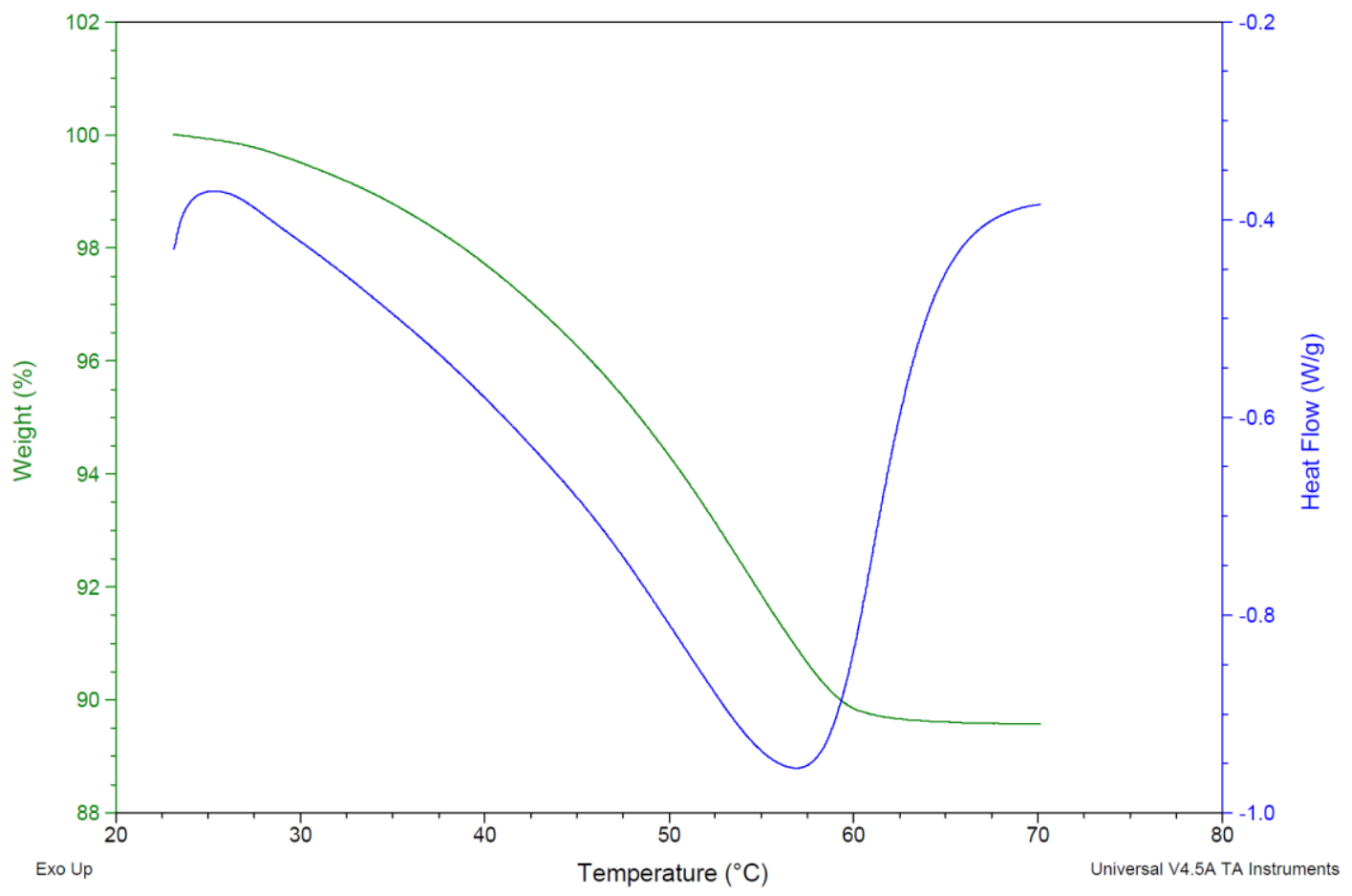

Figure 19: TGA of LAO from 1:5 molar pre-cursor mix showing the inverse peak at $58^{\circ} \mathrm{C}$ 


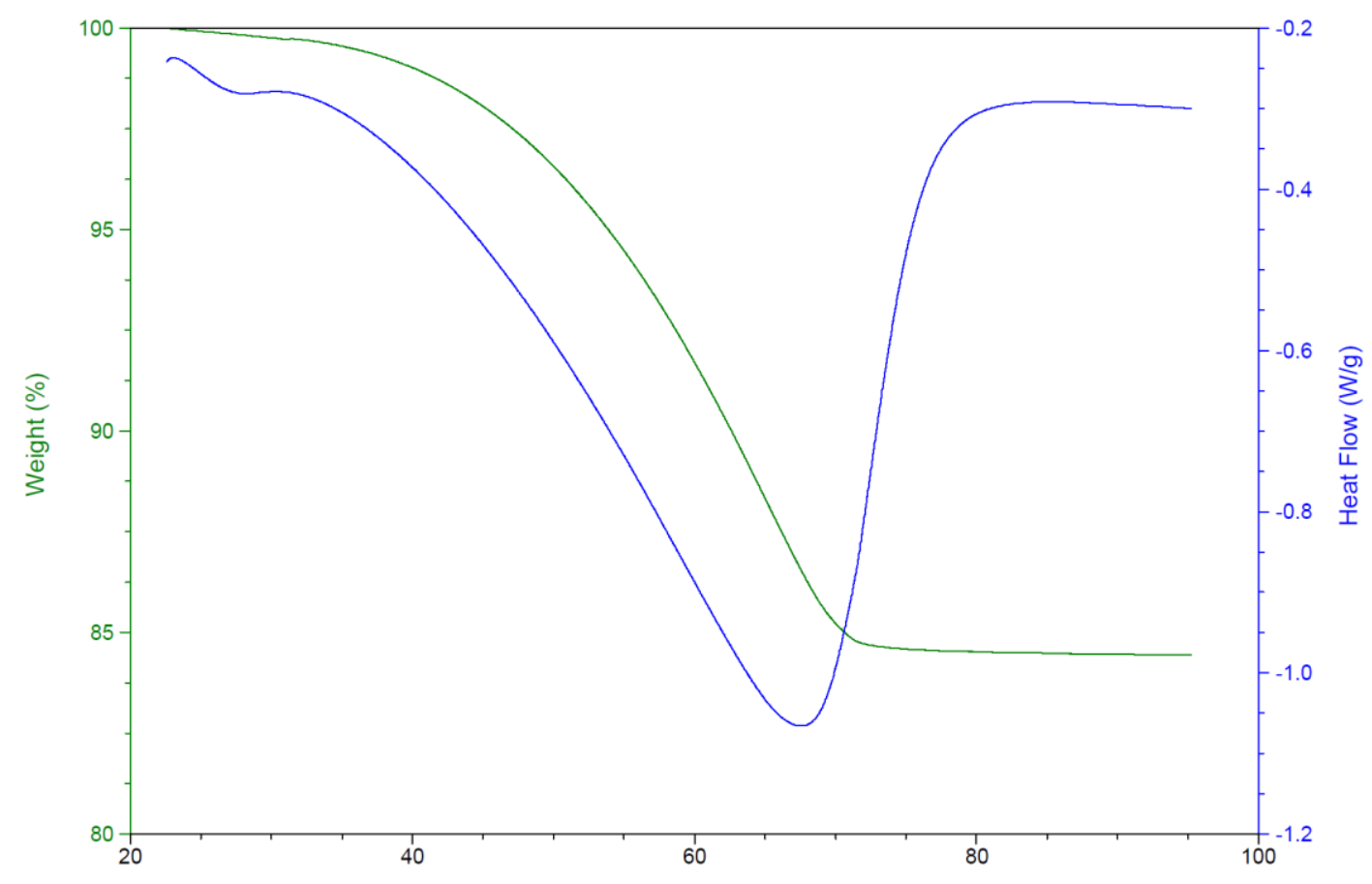

Figure 20: TGA of LAO from 1:6 molar pre-cursor mix showing the inverse peak at $68^{\circ} \mathrm{C}$

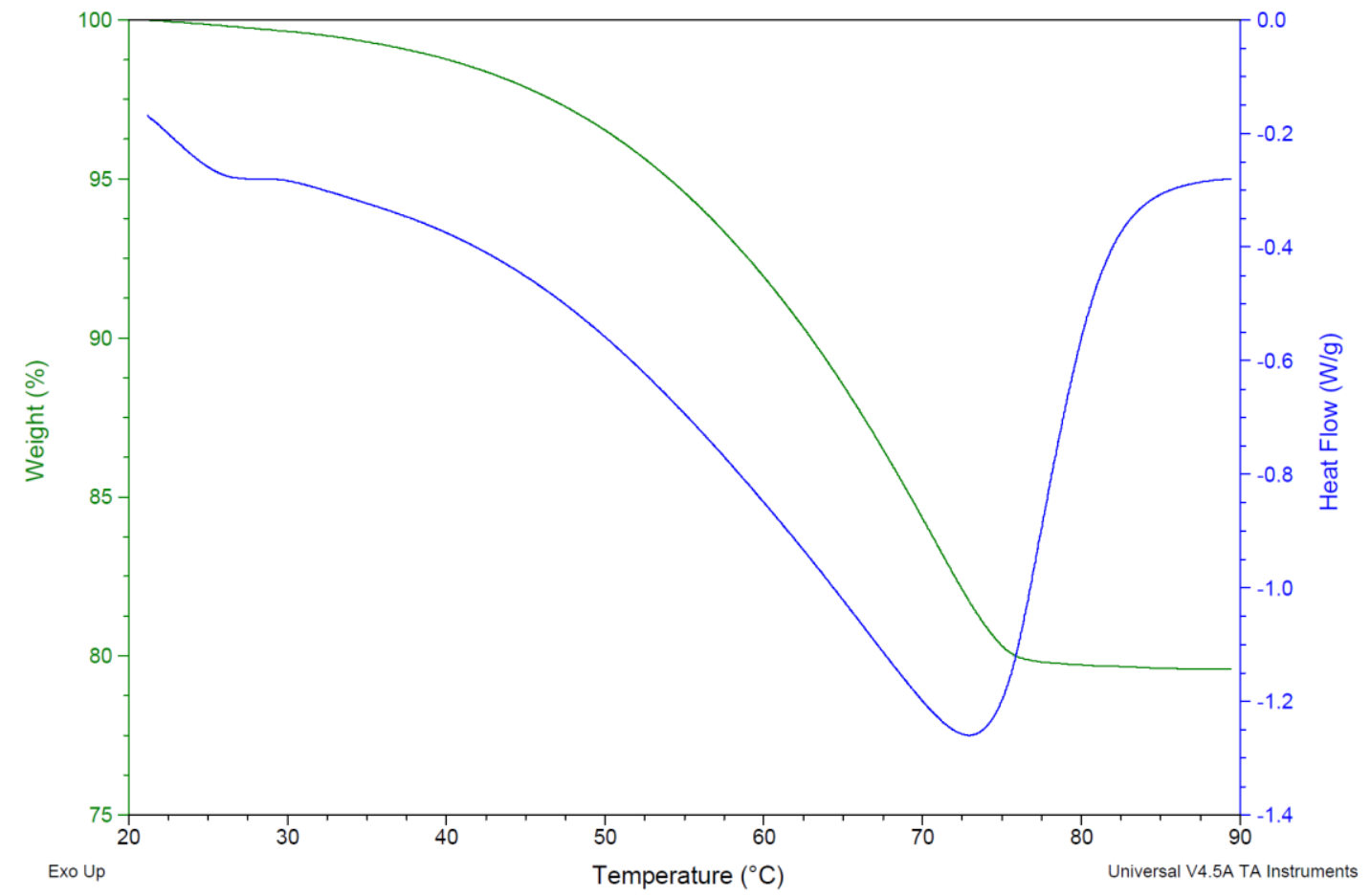

Figure 21: TGA of LAO from 1:7 molar pre-cursor mix showing the inverse peak at $72^{\circ} \mathrm{C}$ 
All further experiments were performed with a 1:6 molar mix. While Table 3 suggests that $1: 7$ has a higher adsorption of water over 1:6, we preferred 1:6 for two reasons: one, the cost of Lithium Hydroxide required us to minimize its usage, and two, the knee temperature was lower $\left(68^{\circ} \mathrm{C}\right.$ vs $\left.72^{\circ} \mathrm{C}\right)$, which makes this more suitable for solar thermal sources of heat.

To re-validate the need for excess lithium as part of the chemisorption, the synthesized nano LAO was washed in water to remove the excess $\mathrm{LiOH}$, dried, lightly powdered in a pestle and analyzed by TGA. Figure 22, 23 and 24 are the TGA results for samples that were subject to different wash and dry techniques, which show how washing off the excessive $\mathrm{LiOH}$ result in progressively reduced desiccant characteristics.

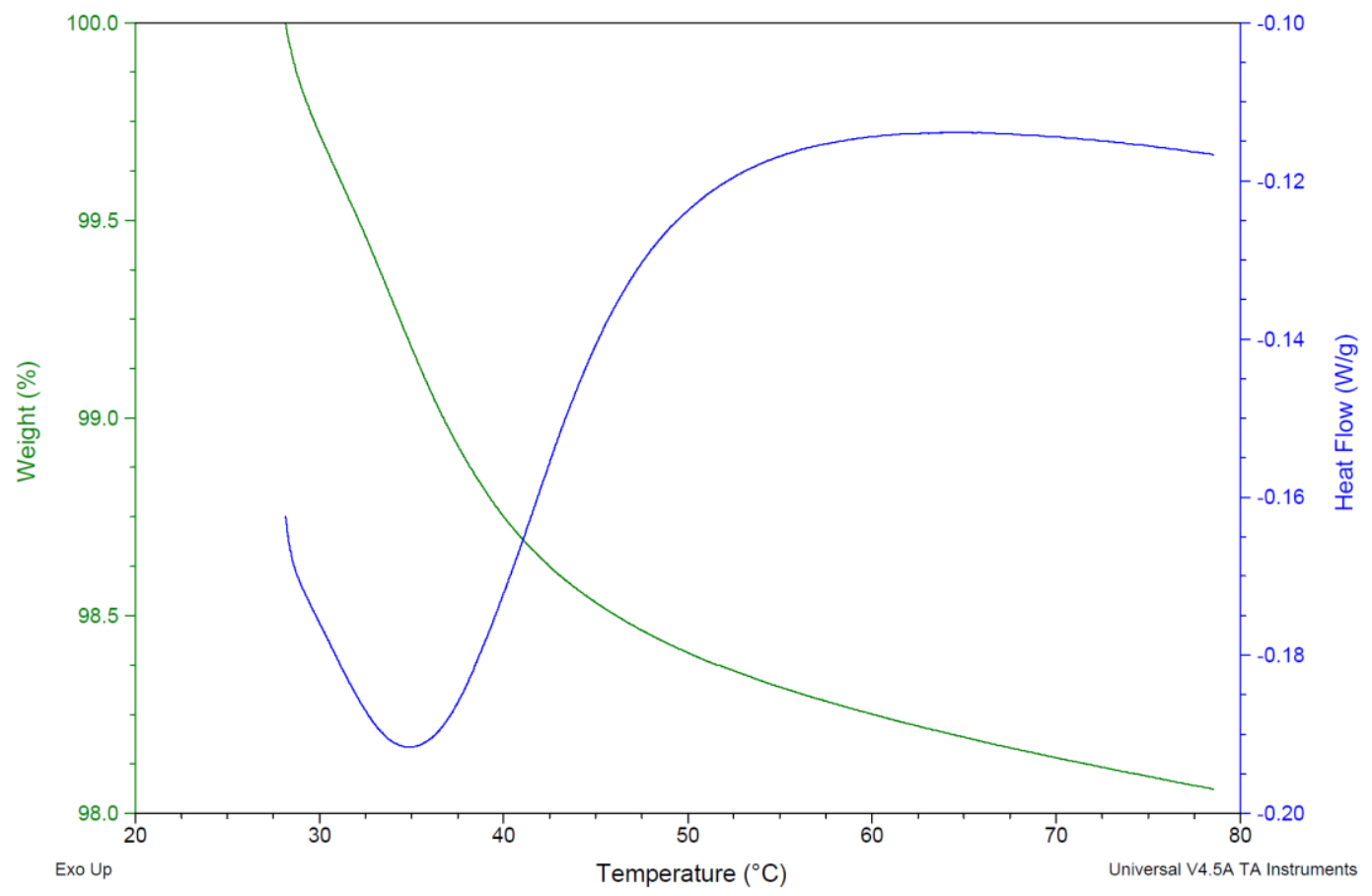

Figure 22: TGA of partially washed 1:6 nano LAO (typical results) showing the inverse peak at $35^{\circ} \mathrm{C}$ and $<2 \%$ desorption 


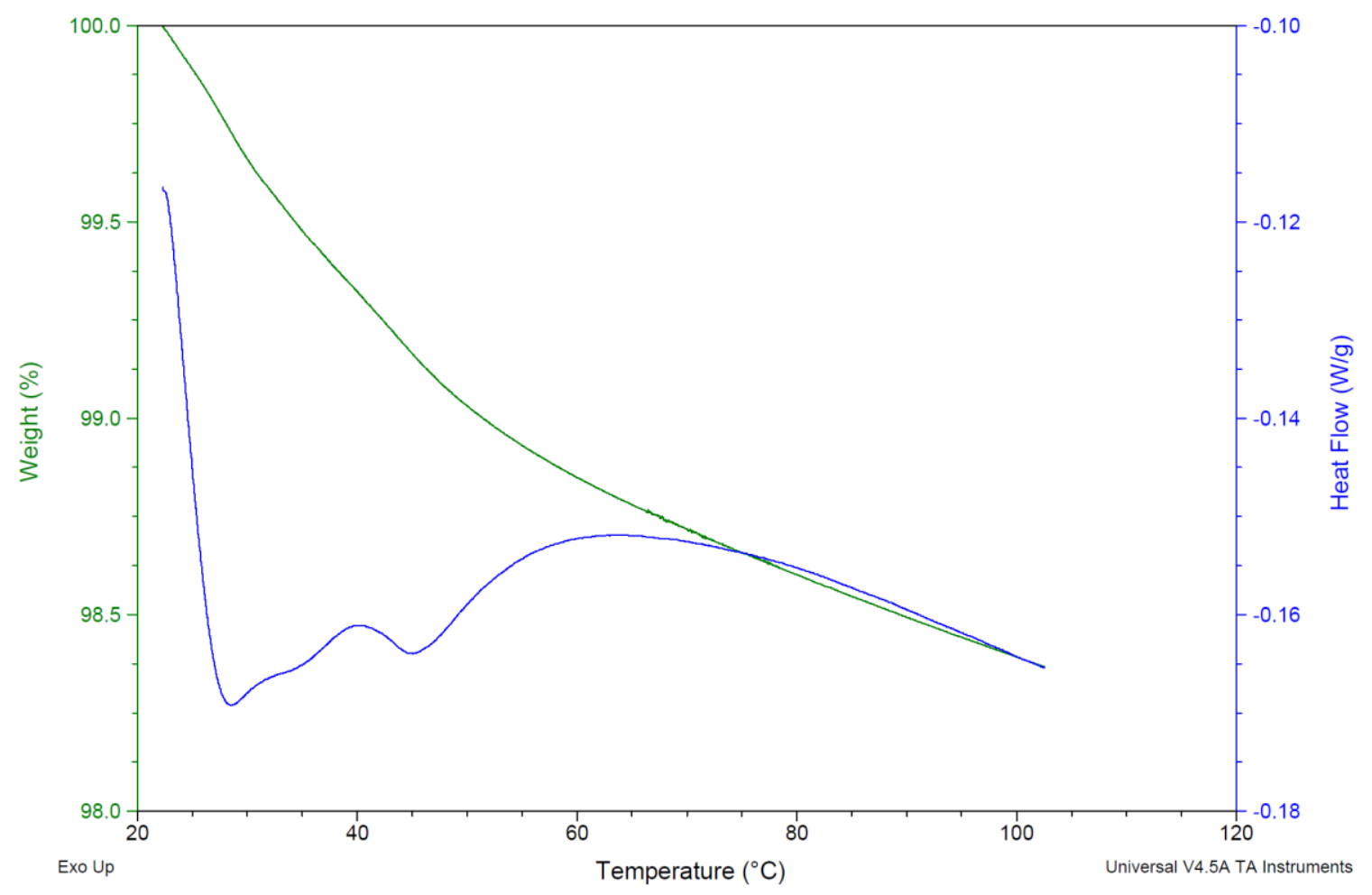

Figure 23: TGA of twice washed 1:6 nano-LAO, vacuum dried, that does not show the characteristic desiccant curves

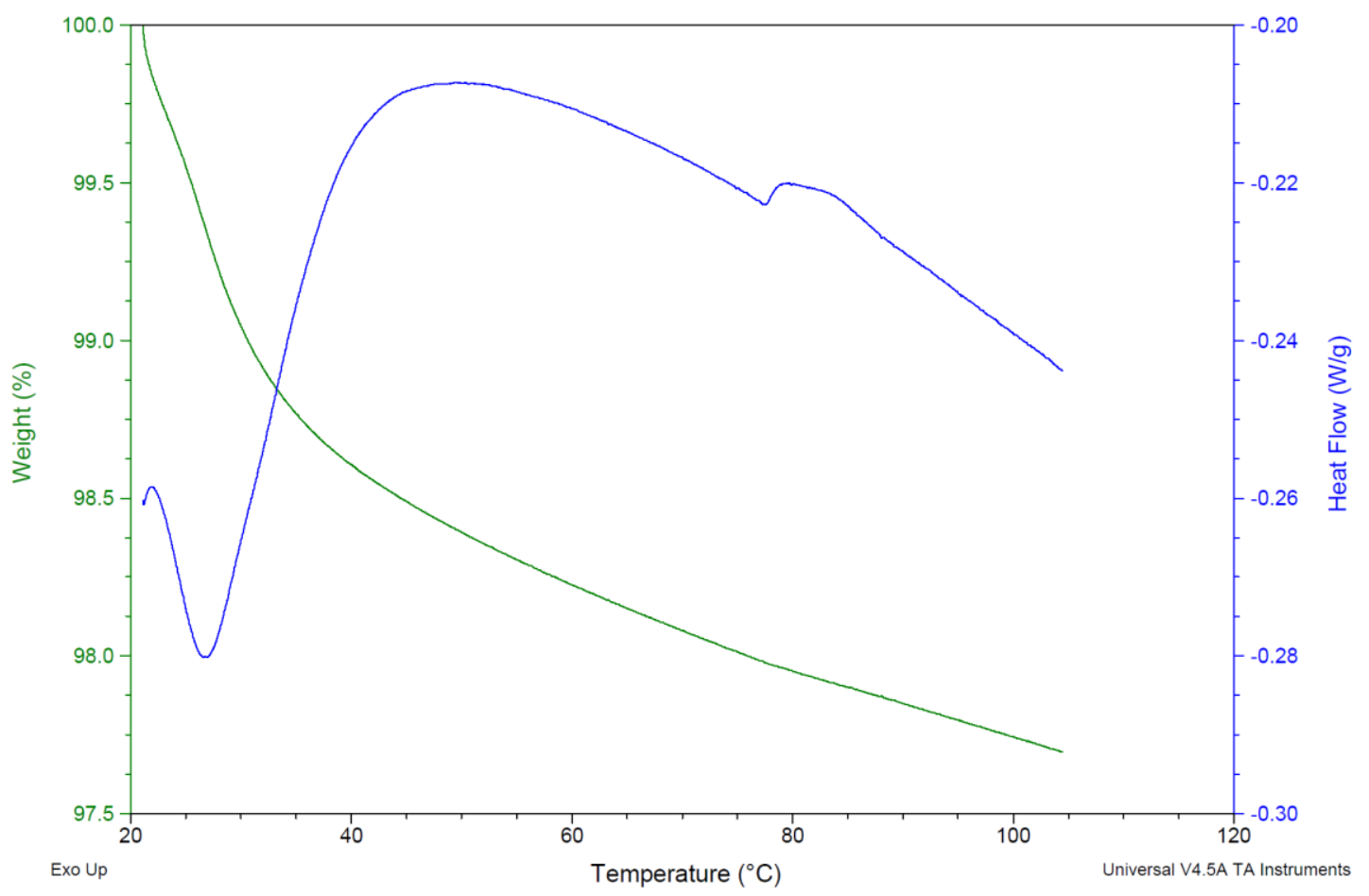

Figure 24: TGA of single washed 1:6 nano-LAO, dried at $120^{\circ} \mathrm{C}$, that does not show the characteristic desiccant curves 
We see an extremely marked reduction $(\sim 16 \% \mathrm{w} / \mathrm{w}$ to $1.8 \% \mathrm{w} / \mathrm{w})$ of water desorption. This confirms the need for the presence of $\mathrm{LiOH}$ to aid the chemisorption of water. The next step in material characterization required was to test the adsorb/desorb kinetics. The following approaches were validated:

Direct synthesis on aluminum sheets - During adsorption the desiccant heats up (the latent heat of vaporization). This heat would need to be taken away and potentially reused. Coating the desiccant on a thermally conducting substance, similar to coated desiccant wheels illustrated in Fig 15 , is one possible method to achieve this. Fig 25 is envisaged as a possible manufacturing process, if this synthesis on metal was successful. This illustrates a continuous coating and synthesis process - a metal sheet in run through a tray containing the precursor mix. The sheet is then subject to plasma on both sides, causing the synthesis of nano LAO on both surfaces. 


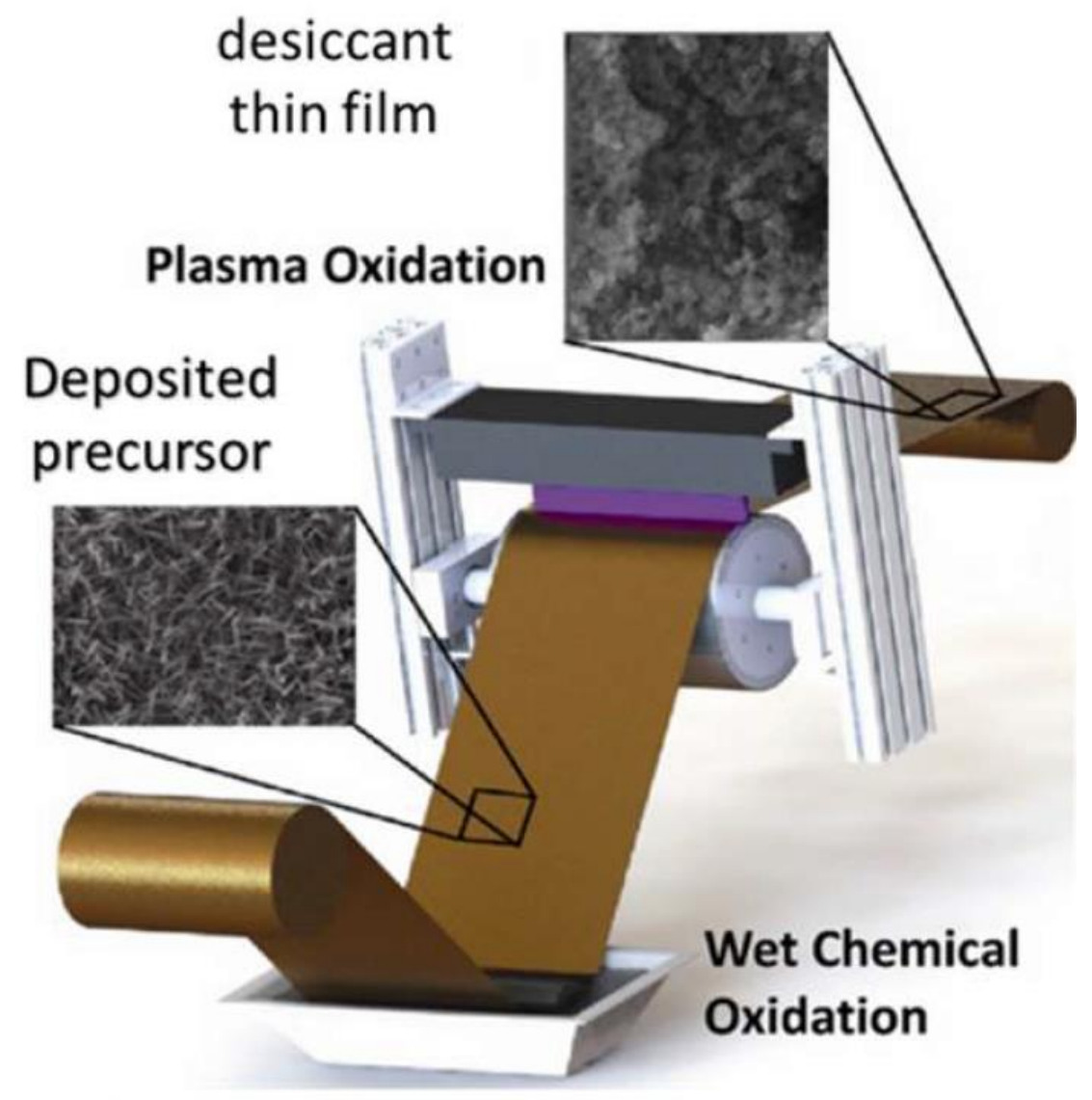

Figure 25: Proposed direct synthesis of LAO on metal foil where the sheet is coated in a dip tray and immediately subject to plasma oxidation

In this investigation, $0.125 \mathrm{~mm}$ aluminum sheets were coated with 1:6 molar ratio materials and subject to the plasma technique described earlier.

A critical observation was that the time taken to coat the sheet with the precursor paste prior to the plasma synthesis had to be extremely short, just a few seconds ( $<10$ seconds), as otherwise the aluminum sheet would rapidly corrode and disintegrate, and we could then not hold the sheet over the plasma flame. Thermal synthesis failed for every temperature setting, as even $1.25 \mathrm{~mm}$ sheets would crumble to powder, but the SolvoPlasma ${ }^{\mathrm{TM}}$ method worked without 
corroding even $0.01 \mathrm{~mm}$ thin foil. Fig 26 is the SEM analysis of the on-substrate direct synthesis LAO, which clearly shows 2D 'clusters of wires' morphologies.

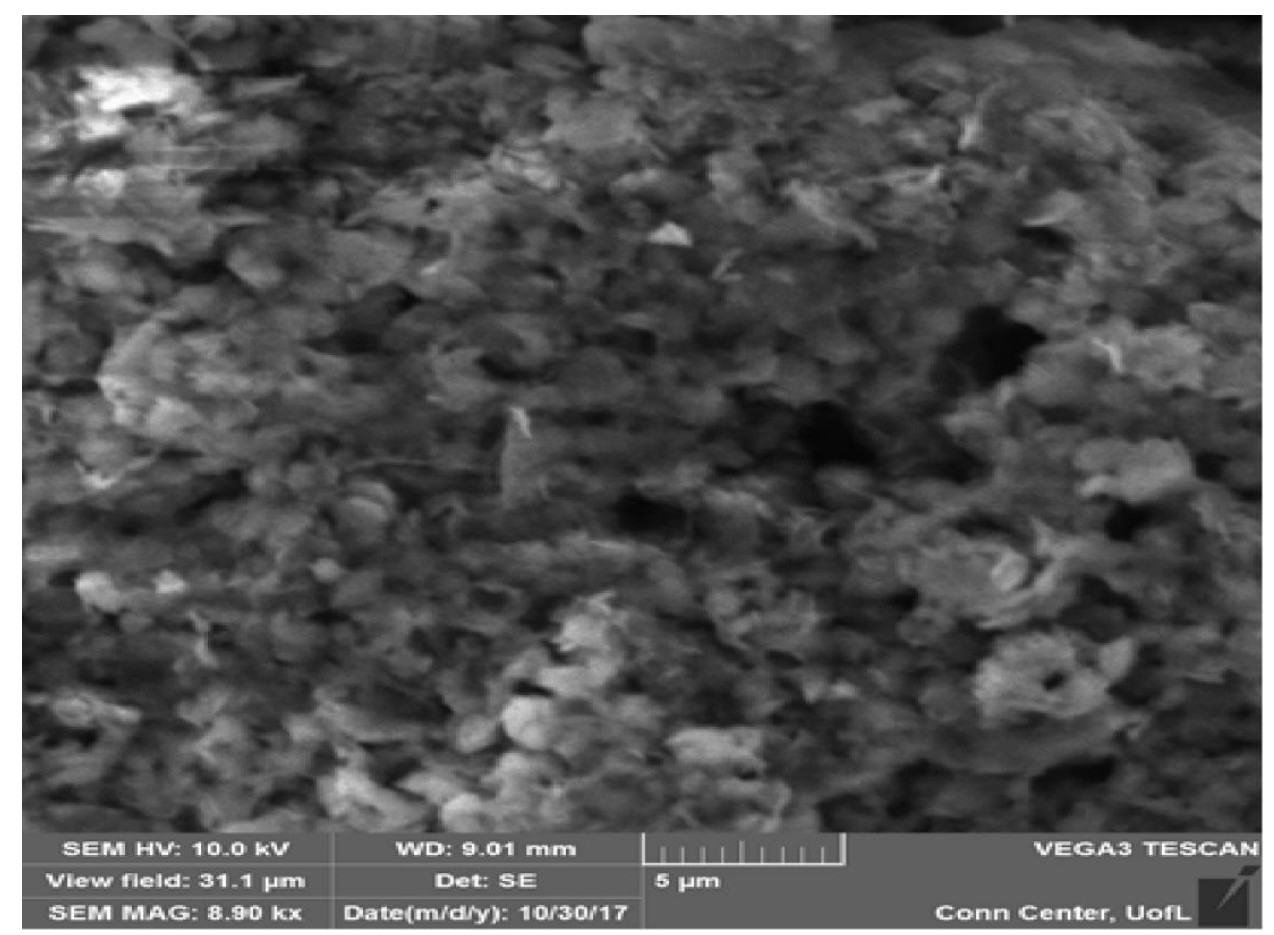

Figure 26: SEM of on-aluminum synthesized LAO showing nano fibres

The kinetics on adsorption and desorption are tabulated in Table 4 (coating thickness of $11.5 \mathrm{~g} / \mathrm{m}^{2}$ ) and Table 5 (coating thickness of $2 \mathrm{~g} / \mathrm{m}^{2}$ ). The ambient temperature is $23^{\circ} \mathrm{C}, \mathrm{RH}$ is $60 \%$, and the desorption is at $86^{\circ} \mathrm{C}$. Time for adsorption and desorption is 120 seconds each.

\begin{tabular}{|c|c|c|c|c|c|c|c|}
\hline Status & Run 1 & Run 2 & Run 3 & Run 4 & Run 5 & Run 6 & Run 7 \\
\hline After adsorb (grams) & 15.9846 & 15.9845 & 15.9844 & 15.9845 & 15.9846 & 15.9844 & 15.9844 \\
\hline After desorb (grams) & 15.9823 & 15.9823 & 15.9824 & 15.9825 & 15.9823 & 15.9826 & 15.9826 \\
\hline Wt change (grams) & 0.0023 & 0.0022 & 0.002 & 0.002 & 0.0023 & 0.0018 & 0.0018 \\
\hline $\begin{array}{l}\% \text { wt change from } \\
\text { initial sample weight }\end{array}$ & 6.03 & 5.77 & 5.24 & 5.24 & 6.03 & 4.72 & 4.72 \\
\hline
\end{tabular}

Table 4: Direct synthesis on aluminum foil, $11.5 \mathrm{~g} / \mathrm{m}^{2}, 120 \mathrm{sec}$ adsorption +120 secdesorption@86 


\begin{tabular}{|c|c|c|c|c|c|c|c|}
\hline Status & Run 1 & Run 2 & Run 3 & Run 4 & Run 5 & Run 6 & Run 7 \\
\hline After adsorb (grams) & 15.7265 & 15.7264 & 15.7263 & 15.7261 & 15.7263 & 15.7263 & 15.7264 \\
\hline After desorb (grams) & 15.7246 & 15.7247 & 15.7245 & 15.7243 & 15.7245 & 15.7246 & 15.7243 \\
\hline Wt change (grams) & 0.0019 & 0.0017 & 0.0018 & 0.0018 & 0.0018 & 0.0017 & 0.0021 \\
\hline$\%$ wt change from & & & & & & & \\
\hline initial sample weight & 27.14 & 24.29 & 25.71 & 25.71 & 25.71 & 24.29 & 30.00 \\
\hline
\end{tabular}

Table 5: Direct synthesis on aluminum foil, $2 \mathrm{~g} / \mathrm{m}^{2}, 120 \mathrm{sec}$ adsorption + $120 \mathrm{sec}$ desorption@86C

Thinner coatings were attempted but abandoned as the weight of the coating was too small and even with four decimal places to the gram of our weighing scale, the measurements were unreliable.

We observe the substantial increase (from an average of $5.39 \%$ to $26.12 \%$ ) in the adsorption with a thinner layer of the desiccant. Irrespective of the difference in the performance based on the thickness of coating we conclude that this desiccant is extremely promising for coated desiccant wheels. Fig 27 compares these results along with error bars.

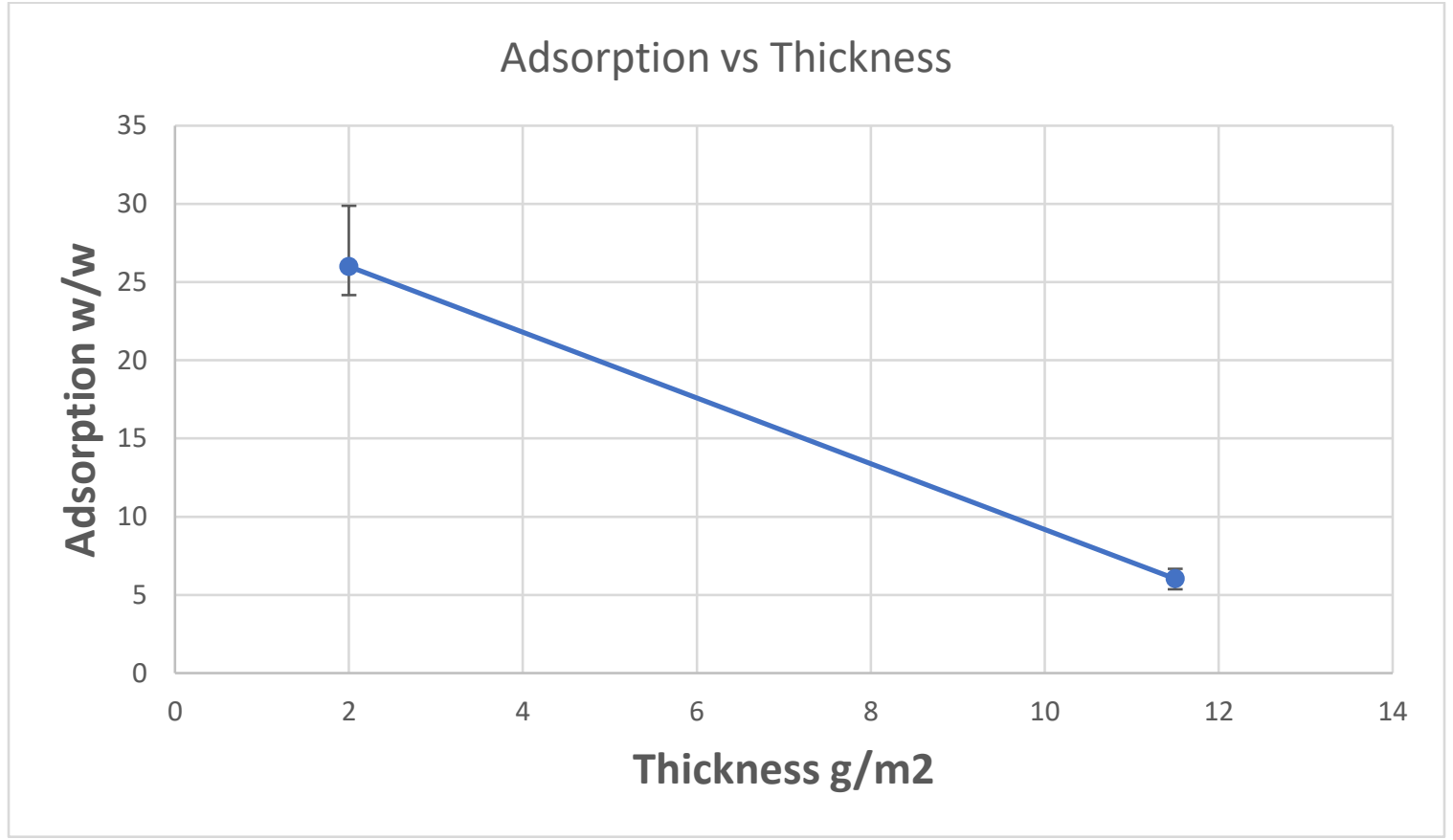

Figure 27: Adsorption vs Coating Thickness, with error bars 
No further research was done by this author in this area - the conclusion that was taken away from these experiments was that the kinetics are extremely fast, in the order of minutes, instead of hours for the commercial desiccants that are presently available. Table 6 lists the time taken for adsorption compared to a few commercial desiccants [77].

\begin{tabular}{|l|r|r|l|}
\hline Desiccant & Capacity \%w/w & Time & Units \\
\hline Silica Gel & 33 & 5.5 & Hours \\
\hline Bentonite Clay & 25 & 7 & Hours \\
\hline Molecular Sieve & 21 & 3 Hours \\
\hline Calcium Sulphate & 10 & 4 & Hours \\
\hline Calcium Oxide & 5 & 10 Hours \\
\hline LAO & 16 & 120 Seconds \\
\hline
\end{tabular}

Table 6: Time taken for adsorption (75\%RH, 25 C) [76] showing the extremely favorable kinetics of LAO vs some commercial desiccants

Increasing the thickness of coating without reducing the \% adsorb/desorb is desirable to enable us to load more desiccant on the same surface area. One approach that was tried was to add other materials to the precursor mix, that would increase the porosity of the coating. Of the several different materials that we tried, Triton-X, a laboratory-grade detergent, was the only one that demonstrated desorption with thicker layers (as much as $20 \mathrm{~g} / \mathrm{m}^{2}$ ). Fig 28 shows the TGA for on-glass synthesis with 0.02 mMole of TritonX-114 - we can see that the desorption has come down from $16 \% \mathrm{w} / \mathrm{w}$ to $12 \% \mathrm{w} / \mathrm{w}$. This is still substantially better than the $\sim 5 \%$ that we observe in Table $4\left(11.5 \mathrm{~g} / \mathrm{m}^{2}\right)$ - future 
research on the use of Triton-X in the pre-cursors for on-foil direct synthesis is therefore warranted.

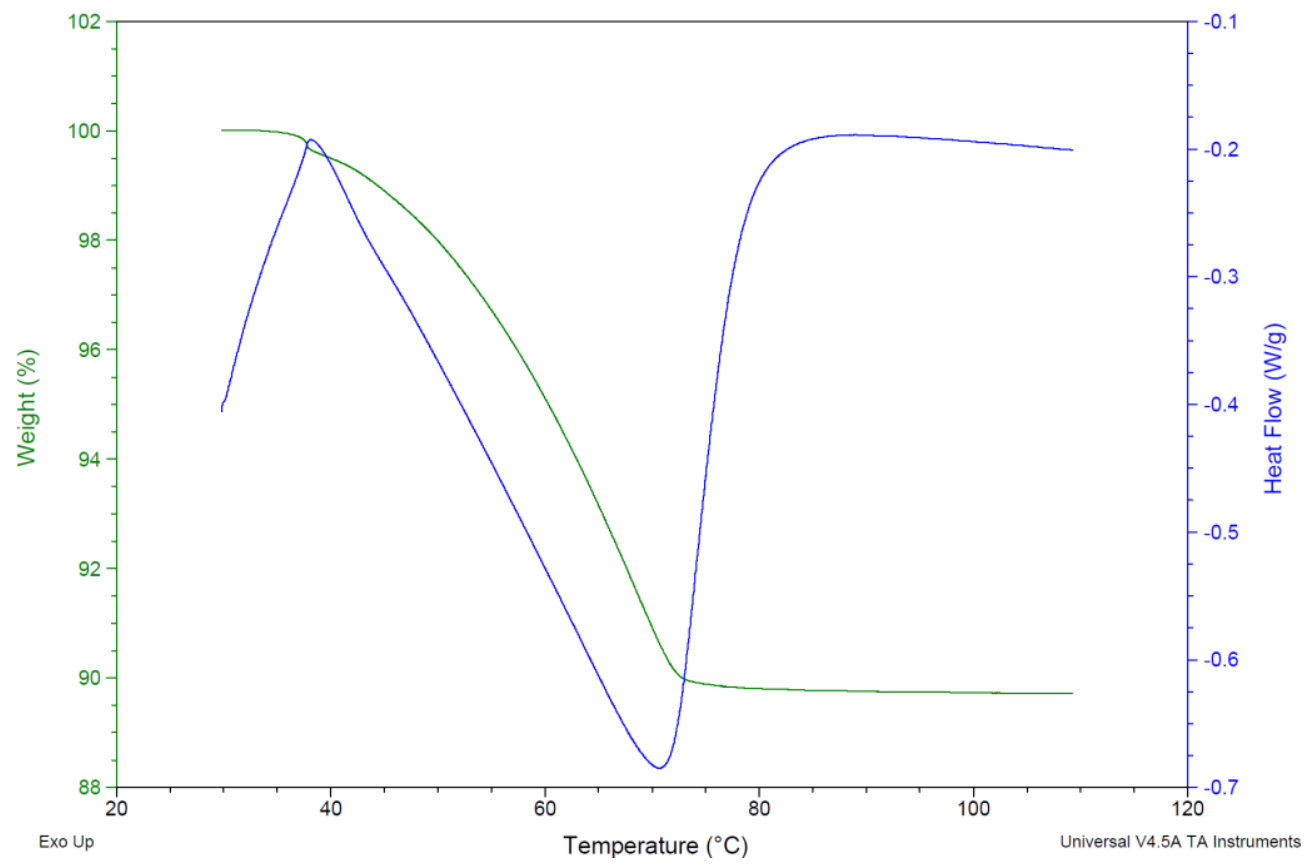

Figure 28: TGA: On-glass synthesis with TritonX-114, + sinter @ 400C showing $\sim 12 \%$ desorption capacity at $70^{\circ} \mathrm{C}$

Coatings - We next looked at coating material with the desiccant after synthesis, as this could be a preferred mode for several applications. The precursor material is coated with a roller or sprayed on a conveyor which is then subject to a downstream plasma flame and optionally a flame source, after which the synthesized nano material is scraped and collected in a reservoir.

Synthesized nano LAO was crushed into fine powder and mixed with (a) 1-Methoxy, 2-Propanol and (b) Methanol. Both pastes were applied to glass slides, heated to $100^{\circ} \mathrm{C}$, and different slides sintered to $500^{\circ} \mathrm{C}$ and $900^{\circ} \mathrm{C}$. It was observed that the adsorption and desorption reduced substantially $(<3 \%)$ and the X-Ray Diffraction (XRD) analysis showed significant shifts, indicating change 
in the material composition - that there was a chemical change in the Lithium Aluminate (Fig $29 \& 30$ ). We therefore concluded that further research was needed to be done on the process of using post-synthesis coatings.

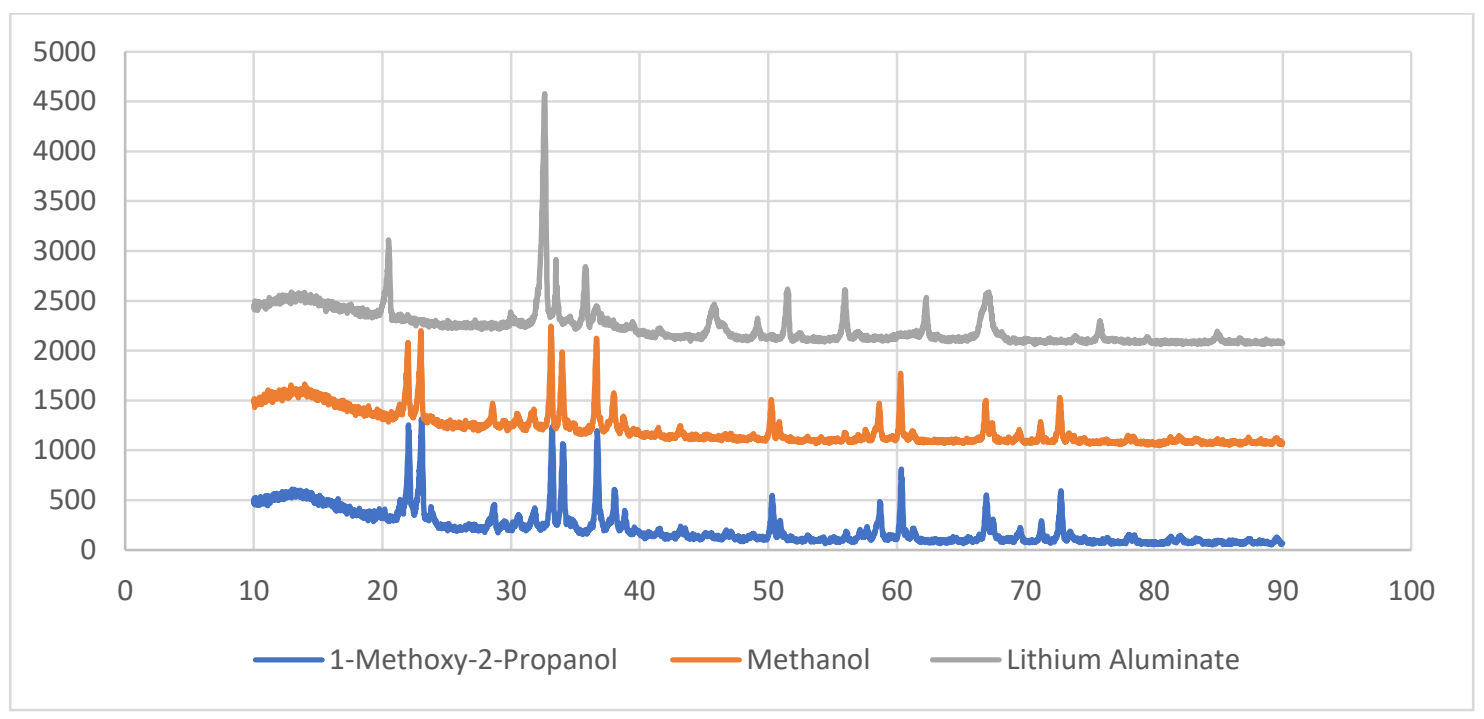

Figure 29: XRD of $L A O$ coatings, sintered @ $550^{\circ} \mathrm{C}$ that show chemical changes due to the process employed

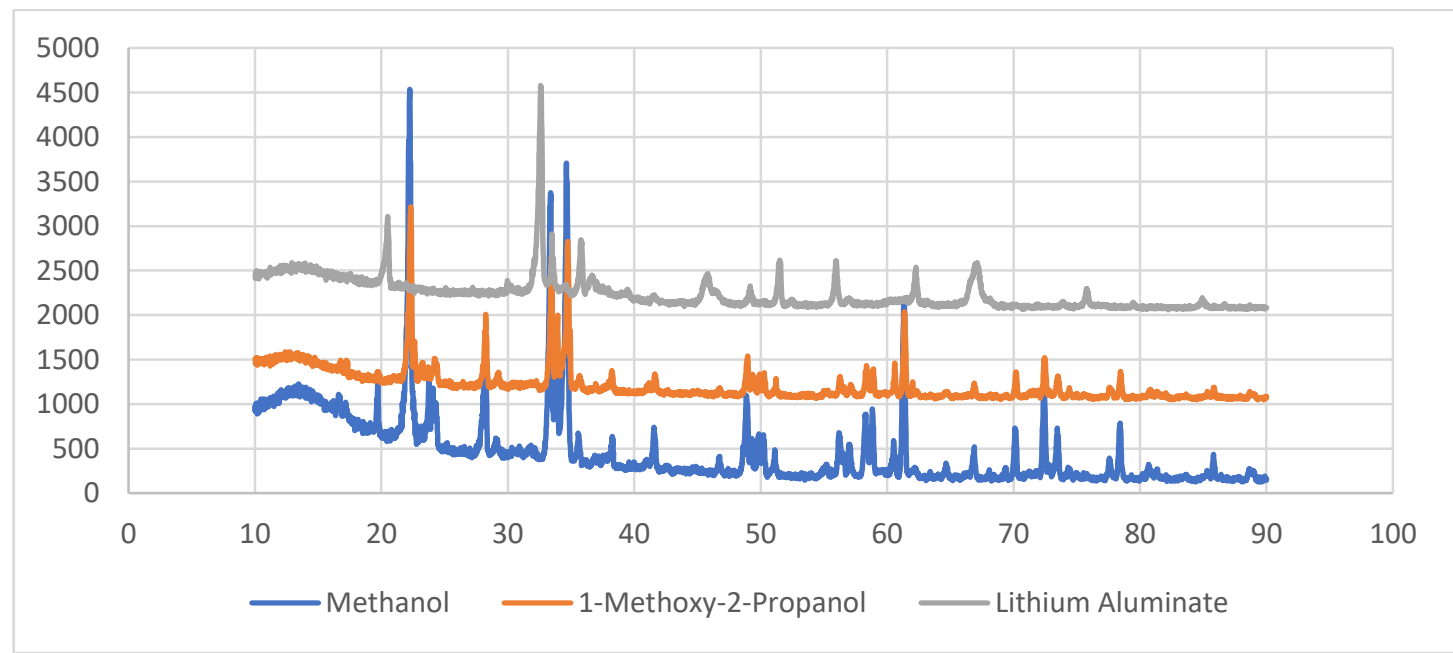

Figure 30: XRD of LAO coatings, sintered@900 C also showing chemical changes

Parallel experiments to coat corderite honeycomb structures, with waterbased suspensions, 1-Methoxy 2-Propanol and with Methanol, had all three 
showing no measurable adsorb/desorb values. The advantage of using corderite honeycombs are many, and further research on loading these with synthesized LAO is a promising research area, though we did not succeed in our specific tests.

Aggregates: While films on metal foil were successful, we reasoned that loading large quantities (we would need several kilograms of material) of LAO with surface thicknesses of $2 \mathrm{~g} / \mathrm{m}^{2}$ would result in extremely heavy and large rotating systems. These would make the final system complex and expensive to maintain. We therefore wanted the LAO to be available in an extrudate form, which will allow us to pack the large quantity of LAO into a small chamber, through which ambient air would be blown for adsorption, and heated air for desorption.

Appendix 1 details our research to develop aggregates in an extrudate form. We failed in these attempts - all extrudates showed zero capacity.

From our experiments we therefore conclude the following: The nano form of Lithium Aluminate (LAO) synthesized using the facile and patented SolvoPlasma ${ }^{\mathrm{TM}}$ method meets the desiccant properties listed in Table 2. This table is reproduced below, as Table 6 , indicating the parameters that were tested and found suitable:

\begin{tabular}{|l|l|l|}
\hline Specification & Range / limit required & Validated? \\
\hline $\begin{array}{l}\text { Adsorption } \\
\text { capacity }\end{array}$ & $\sim \mathbf{2 0 \%} \mathbf{w} \mathbf{w}$ & Yes \\
& $\begin{array}{l}\text { As the kinetics we are aiming for } \\
\text { are fast, in minutes, the heat of } \\
\text { adsorption will cause a rapid rise }\end{array}$ & $\begin{array}{l}\text { Capacity was found to be } \\
16 \% \text { for 1:6 precursor }\end{array}$ \\
\hline
\end{tabular}




\begin{tabular}{|c|c|c|}
\hline & $\begin{array}{l}\text { in the desiccant temperature. We } \\
\text { also require a low desorption } \\
\text { temperature. The specification is } \\
\text { therefore set for a relatively low } \\
\text { adsorption capacity. }\end{array}$ & $\begin{array}{l}\text { molar ratio, and } 21 \% \text { for } \\
1: 7\end{array}$ \\
\hline $\begin{array}{l}\text { Desorption } \\
\text { temperature }\end{array}$ & $\begin{array}{l}>60^{\circ} \mathrm{C}<70^{\circ} \mathrm{C} \\
\text { Ambient temperatures in most } \\
\text { parts of the tropics reach } \sim 40^{\circ} \mathrm{C} \text {. } \\
\text { The heat of adsorption will raise } \\
\text { the desiccant temperature. Too } \\
\text { low a desorption temperature will } \\
\text { result in poor adsorption. } \\
\text { The target setup uses flat plate } \\
\text { solar thermal air heaters. } \\
\text { Typically, these deliver no more } \\
\text { than } 90^{\circ} \mathrm{C} \text {. Desorption causes } \\
\text { this temperature to fall. We } \\
\text { therefore need the desorption } \\
\text { temperature to be lower than this. }\end{array}$ & $\begin{array}{l}\text { Yes } \\
\text { The 1:6 mix has a } \\
\text { desorption temperature } \\
\text { of } 68^{\circ} \mathrm{C}\end{array}$ \\
\hline Toxicity & Negative & $\begin{array}{l}\text { Yes } \\
\text { As per literature }\end{array}$ \\
\hline Corrosive & Negative & $\begin{array}{l}\text { Yes } \\
\text { As per literature }\end{array}$ \\
\hline $\begin{array}{l}\text { Kinetics } \\
\text { (adsorb / } \\
\text { desorb) }\end{array}$ & $\begin{array}{l}\text { Minutes and not hours } \\
\text { A simple and compact system will } \\
\text { deliver higher water output if } \\
\text { multiple cycles can be run during } \\
\text { the approximately } 5 \text { hours of } \\
\text { sunshine. The more the cycles } \\
\text { we can run, the more the output. } \\
\text { Here we are assuming that the } \\
\text { area needed (the real estate) for } \\
\text { the flat panel solar thermal } \\
\text { collectors are not the limiting } \\
\text { factor in the system design. }\end{array}$ & $\begin{array}{l}\text { Yes } \\
\sim 120 \text { seconds for } \\
\text { adsorption and } \sim 120 \\
\text { seconds for desorption } \\
\text { when tested in powder } \\
\text { form as well as on-foil } \\
\text { direct synthesis }\end{array}$ \\
\hline Life & $\begin{array}{l}\sim 20 \text { years } \\
\text { Longer life desiccants imply lower } \\
\text { capital and operating costs. }\end{array}$ & Not validated \\
\hline Form & Solid & Yes \\
\hline
\end{tabular}




\begin{tabular}{|l|l|l|}
\hline & $\begin{array}{l}\text { Simple, easy to maintain systems } \\
\text { are best managed with solid } \\
\text { desiccants. }\end{array}$ & $\begin{array}{l}\text { The material is a solid } \\
\text { powder }\end{array}$ \\
\hline $\begin{array}{l}\text { Absorption } \\
\text { Isotherm }\end{array}$ & $\begin{array}{l}\text { Langmuir } \\
\text { The desiccant must perform well } \\
\text { and deliver substantially the } \\
\text { same water output irrespective of } \\
\text { the RH, as well as work well in } \\
\text { desert conditions. }\end{array}$ & $\begin{array}{l}\text { Not fully tested } \\
\text { During tests we did not } \\
\text { observe a significant } \\
\text { change in the adsorption } \\
\text { teme with changing RH. } \\
\text { However, this was not } \\
\text { systematically tested }\end{array}$ \\
\hline Material & Inorganic, earth abundant & $\begin{array}{l}\text { Yes } \\
\text { Both for long life and cost factors. }\end{array}$ \\
& $\begin{array}{l}\text { Lithium is the 25 } 25^{\text {th }} \text { most } \\
\text { abundant material, and } \\
\text { while not considered } \\
\text { earth abundant, it is not a } \\
\text { rare earth material either. } \\
\text { Aluminum comprises } \\
\text { about 8\% of the Earth's } \\
\text { crust. }\end{array}$ \\
\hline
\end{tabular}

Table 7: Summary of results, as compared to the ideal desiccant

The lower operating temperature is significant is several respects:

- A lower temperature would mean significant savings in the energy required to heat air and the desiccant compared to a desiccant that desorbs at a higher temperature. While we do not save on energy requirements for the latent heat of vaporization, we do save on the sensible heat needed for air and the desiccant. The energy needed to heat any substance is $\mathrm{mC}_{p} \mathrm{dT}$, where $\mathrm{m}$ is the mass, $\mathrm{C}_{\mathrm{p}}$ is the heat capacity, and dT the change in temperature of the substance. Here dT is decreased, implying that the amount of heat necessary is also decreased. 
- A lower temperature has far more significant saving due to lower thermal losses of the overall system.

- Systems powered by solar thermal have lower radiative losses when these are operated at lower temperatures.

- A lower temperature of the desorption stream however implies a lower water carrying capacity (see Fig 4) of colder air. For the same amount of water condensed, we would therefore require larger amounts of air to be heated and sent through the desiccant. This will mean higher energy consumption for the blowers. Cooling this air will also require far more efficient, large and power intensive condensers as the temperature difference with the ambient air (used for cooling) is quite small $\left(30-40^{\circ} \mathrm{C}\right)$.

We can theoretically arrive at expected energy savings from using a lower temperature desiccant $\left(68^{\circ} \mathrm{C}\right.$ for LAO vs $118^{\circ} \mathrm{C}$ for Silica Gel). Here the only parameter we consider is the temperature, and how this impacts efficiency (we ignore all other parameters like thermal losses of systems operating at higher temperatures, which if considered will show an increase in thermal efficiency of LAO). From Table 8 we arrive at an efficiency improvement of $43 \%$, considering energy required for heating air (heating air by $41^{\circ} \mathrm{C}$ for LAO vs $91^{\circ} \mathrm{C}$ for Silica Gel), heating the water in the desiccant (m Cp dT of $171.63 \mathrm{KJ}$ for LAO vs $380.93 \mathrm{KJ}$ for Silica Gel) and solar thermal conversion efficiency ( $45 \%$ for Flat Plate @ $68^{\circ} \mathrm{C}$ vs $32 \%$ for Concentrated Solar @ $\left.118^{\circ} \mathrm{C}\right)$. Were we to ignore the 
efficiency of the solar thermal collectors and consider energy requirement only, we still arrive at an improved efficiency of $19.5 \%$.

\begin{tabular}{|c|c|c|}
\hline Parameter & LAO & Silica Gel \\
\hline Desorb Temperature ${ }^{\circ} \mathrm{C}$ & 68 & 118 \\
\hline Specific heat capacity or air J/g C & 0.933 & 0.933 \\
\hline Specific heat capacity or water J/g C & 4.186 & 4.186 \\
\hline Ambient temperature ${ }^{\circ} \mathrm{C}$ & 27 & 27 \\
\hline Latent heat of evaporation Water kJ/g C & 2.264 & 2.264 \\
\hline Air heated by ${ }^{\circ} \mathrm{C}$ & 41 & 91 \\
\hline Energy to heat $10 \mathrm{Kg}$ air $\mathrm{KJ}$ & 382.53 & 849.03 \\
\hline $\begin{array}{l}\text { Energy to desorb } 1 \mathrm{Kg} \text { water from desiccant } \mathrm{KJ} \text { ( } 10 \% \text { desorption from } \\
10 \mathrm{Kg} \text { of desiccant) } \mathrm{KJ}\end{array}$ & 2264.00 & 2264.00 \\
\hline $\begin{array}{l}\text { Energy required to heat water from ambient to desorption } \\
\text { temperature ( } m \mathrm{Cp} d T) \mathrm{KJ}\end{array}$ & 171.63 & 380.93 \\
\hline Total for air + desorption + m Cp dT KJ & 2818.16 & 3493.96 \\
\hline Watt Hours & 782.821 & 970.543 \\
\hline Type of solar thermal collector & Flat Plate & Evacuated Tube \\
\hline $\begin{array}{l}\text { Effeciency (typical @ desorption temperature) Data from } \\
\text { solarhotwater-systems.com }\end{array}$ & $45 \%$ & $32 \%$ \\
\hline $\begin{array}{l}\text { Area required in } \mathrm{m} 2 \text { of land (assuming same time required for } \\
\text { desorption, say } 1 \text { hour, for comparision), insolation of } 1000 \mathrm{~W} / \mathrm{m} 2\end{array}$ & 1.74 & 3.03 \\
\hline $\begin{array}{l}\% \text { effeciency improvement, just considering area required for solar } \\
\text { thermal collectors }\end{array}$ & $43 \%$ & \\
\hline
\end{tabular}

Table 8: Thermal Efficiency LAO vs Silica Gel, showing 43\% improvement in energy efficiency using $L A O$

These calculations do not take into account the overall improvement of the complete system due to the ultra-fast kinetics of LAO as we would need a substantially smaller system, and the savings in real estate (estimated to be about $30 \%$, based on solar thermal efficiency of $45 \%$ for Flat Plate @ $68^{\circ} \mathrm{C}$ vs $32 \%$ for Concentrated Solar @ $118^{\circ} \mathrm{C}$ ) , capital and operating costs by using simpler and cheaper direct air flat plate collectors over evacuated tube collectors which also require heat exchangers to transfer heat from the liquid in the collector to air. 
A detailed study to arrive at the system power gains using a low temperature desiccant has not been done, in the absence of the aggregates.

We conclude that there is promise in proceeding with the developing the aggregate form of $L A O$, as nano-LAO meets the core requirements of a low temperature, ultra-fast solid desiccant. We however failed in our attempt to obtain aggregates - the final step in the search for our ideal desiccant. 


\section{FURTHER RESEARCH}

Much needs to be done to take this to commercial readiness:

The desiccant needs to be available in extrudate or other aggregate form. Coatings on aluminum foils were promising. We are however interested in the extrudate approach (beds of desiccant) as the system design is far simpler (no rotating wheels), far more desiccant material can be loaded in a smaller system, and maintenance and operating costs are far lower for desiccant beds.

Detailed temperature studies of the desiccant in adsorb \& desorb are required - as the temperature rise during adsorption is an extremely important aspect in system design

The life of the desiccant needs to be established, including the impact of environmental pollutants, as commercial life is expected to be about 20 years

The control systems for dynamic configuration needs to be modelled and used as input to system design

A "cartridge" system may be needed to hold the desiccant for easy replacement and periodic off-chamber recharge

Detailed studies are required for solar air heating, with or without thermal storage 


\section{CONCLUSION}

The supply of potable water is rapidly becoming a crisis. Desalination is not appropriate for many parts of the world, nor is fossil fuels as energy sources appropriate, given their negative environmental impact. Atmospheric moisture coupled with solar energy (both thermal and photovoltaics) are emerging as an extremely promising combination and allows the direct extraction of water from air.

There are two main challenges that need to be overcome for this combination to be practical. For real-world, distributed, small-scale installations (2,000 liters per day (LPD) - 20,000 LPD) we need rugged, low-maintenance, simple systems (powered by flat panel solar collectors used to heat air). These systems will deliver low temperature air: about $50^{\circ} \mathrm{C}$ more than ambient - typically $80-90^{\circ} \mathrm{C}$. A solid desiccant that can adsorb and desorb rapidly to support multiple cycles during the 5-7 hours that solar energy is available, without using expensive and complex storage systems, is required.

Secondly, humidity, temperature and solar insolation vary widely across any time period - any time of the day or year. The system must support these variables and still perform optimally. 
This research identifies a metal oxide, Lithium Aluminate, as a nanomaterial synthesized using a UofL patented process, that meets all our requirements for an ideal desiccant for use in simple, low cost, solar powered AWG systems: an earth abundant and stable material, non-toxic, non-corrosive, low temperature of desorption, extremely fast kinetics for adsorption and desorption, operating over a wide range of humidity conditions and manufactured in a low-cost facile process.

This desiccant was validated for its low desorb temperature using TGA, kinetics was established by measurements of adsorption and desorption over time scales as low as 120 seconds, surface morphology by SEM; and XRD to identify the synthesized material as Lithium Aluminate. 


\section{REFERENCES}

1. THE MILLENNIUM DEVELOPMENT GOALS. June 9, 2018]; Available from:

http://www.unfoundation.org/what-we-do/issues/mdgs.html.

2. Wang, L.K., et al., Membrane and desalination technologies. Vol. 13. 2008: Springer.

3. American, S. Israel Proves the Desalination Era Is Here. June 11, 2018]; Available from: https://www.scientificamerican.com/article/israel-proves-the-desalination-era-is-here/.

4. Zhou, Y. and R.S. Tol, Evaluating the costs of desalination and water transport. Water resources research, 2005. 41(3).

5. Karnataka State Natural Disaster Monitoring Cell. Available from:

https://www.ksndmc.org/Drought.aspx.

6. River Systems of Karnataka. Available from:

http://waterresources.kar.nic.in/river systems.htm\#map.

7. Rodell, M., I. Velicogna, and J.S. Famiglietti, Satellite-based estimates of groundwater depletion in India. Nature, 2009. 460(7258): p. 999.

8. $\quad$ Farmer Suicides in Karnataka. Instuture of Social \& Economic Change.

9. Postel, S., et al., Drip irrigation for small farmers: A new initiative to alleviate hunger and poverty. Water International, 2001. 26(1): p. 3-13.

10. Carruthers, S., Hydroponics in India. Practical Hydroponics and Greenhouses, 2013(133): p. 16.

11. Association, N.G.W. June 9, 2018]; Available from:

http://www.ngwa.org/Fundamentals/teachers/Pages/information-on-earth-water.aspx.

12. Kim, H., et al., Water harvesting from air with metal-organic frameworks powered by natural sunlight. Science, 2017. 356(6336): p. 430-434.

13. SkyWater June 9, 2018]; Available from: https://islandsky.com/.

14. Wahlgren, R.V., Atmospheric water vapour processor designs for potable water production: a review. Water Research, 2001. 35(1): p. 1-22.

15. Khalil, A., Dehumidification of atmospheric air as a potential source of fresh water in the UAE. Desalination, 1993. 93(1-3): p. 587-596.

16. Harry, B., Dehumidifiers. 1962, Google Patents.

17. Tripathi, A., et al., Atmospheric water generator. International Journal of Enhanced Research in Science, 2016(5): p. 69-72.

18. Narayan, G.P., et al., Thermodynamic analysis of humidification dehumidification desalination cycles. Desalination and Water Treatment, 2012. 16(1-3): p. 339-353.

19. Chehayeb, K.M., et al., Thermodynamic balancing of a fixed-size two-stage humidification dehumidification desalination system. Desalination, 2015. 369: p. 125139.

20. Mistry, K.H., A. Mitsos, and J.H. Lienhard, Optimal operating conditions and configurations for humidification-dehumidification desalination cycles. International Journal of Thermal Sciences, 2011. 50(5): p. 779-789.

21. Prakash Narayan, G., et al., Thermal design of the humidification dehumidification desalination system: An experimental investigation. International Journal of Heat and Mass Transfer, 2013. 58(1-2): p. 740-748.

22. Sharqawy, M.H., et al., Optimum thermal design of humidification dehumidification desalination systems. Desalination, 2014. 349: p. 10-21. 
23. Wu, G., et al., A study on the maximum gained output ratio of single-effect solar humidification-dehumidification desalination. Solar Energy, 2017. 157: p. 1-9.

24. Campos, B.L.d.O., A.O.S.d. Costa, and E.F.d. Costa Junior, Mathematical modeling and sensibility analysis of a solar humidification-dehumidification desalination system considering saturated air. Solar Energy, 2017. 157: p. 321-327.

25. Franchini, G. and A. Perdichizzi, Modeling of a Solar Driven HD (HumidificationDehumidification) Desalination System. Energy Procedia, 2014. 45: p. 588-597.

26. Ghazy, A. and H.E.S. Fath, Solar desalination system of combined solar still and humidification-dehumidification unit. Heat and Mass Transfer, 2016. 52(11): p. 24972506.

27. Giwa, A., et al., Recent advances in humidification dehumidification (HDH) desalination processes: Improved designs and productivity. Renewable and Sustainable Energy Reviews, 2016. 57: p. 929-944.

28. Hamed, M.H., et al., Mathematical and experimental investigation of a solar humidification-dehumidification desalination unit. Desalination, 2015. 358: p. 9-17.

29. Narayan, G.P., et al., The potential of solar-driven humidification-dehumidification desalination for small-scale decentralized water production. Renewable and Sustainable Energy Reviews, 2010. 14(4): p. 1187-1201.

30. Shalaby, S.M., M.A. Bek, and A.E. Kabeel, Design Recommendations for Humidificationdehumidification Solar Water Desalination Systems. Energy Procedia, 2017. 107: p. 270274.

31. Chehayeb, K.M., et al., Use of multiple extractions and injections to thermodynamically balance the humidification dehumidification desalination system. International Journal of Heat and Mass Transfer, 2014. 68: p. 422-434.

32. Narayan, G.P., et al., Thermodynamic balancing of the humidification dehumidification desalination system by mass extraction and injection. International Journal of Heat and Mass Transfer, 2013. 57(2): p. 756-770.

33. Prakash Narayan, G., J.H. Lienhard, and S.M. Zubair, Entropy generation minimization of combined heat and mass transfer devices. International Journal of Thermal Sciences, 2010. 49(10): p. 2057-2066.

34. Kumar, M. and A. Yadav, Comparative study of solar-powered water production from atmospheric air using different desiccant materials. International Journal of Sustainable Engineering, 2016. 9(6): p. 390-400.

35. Wang, J.Y., R.Z. Wang, and L.W. Wang, Water vapor sorption performance of ACF-CaCl 2 and silica gel-CaCl 2 composite adsorbents. Applied Thermal Engineering, 2016. 100: $\mathrm{p}$. 893-901.

36. Wang, J.Y., et al., Experimental investigation on two solar-driven sorption based devices to extract fresh water from atmosphere. Applied Thermal Engineering, 2017. 127: $p$. 1608-1616.

37. William, G.E., M.H. Mohamed, and M. Fatouh, Desiccant system for water production from humid air using solar energy. Energy, 2015. 90: p. 1707-1720.

38. Gad, H.E., A.M. Hamed, and I.I. El-Sharkawy, Application of a solar desiccant/collector system for water recovery from atmospheric air. Renewable Energy, 2001. 22(4): p. 541556.

39. Tongue, S., Water-from-air system using desiccant wheel and exhaust.

40. Water Maker India. June, 10 2018]; Available from:

http://watermakerindia.com/product.php. 
41. Yuan, Y., et al., Inorganic composite sorbents for water vapor sorption: A research progress. Renewable and Sustainable Energy Reviews, 2016. 54: p. 761-776.

42. Zheng, X., et al., Performance study of composite silica gels with different pore sizes and different impregnating hygroscopic salts. Chemical Engineering Science, 2014. 120: p. 19.

43. Wu, X.N., et al., Review on substrate of solid desiccant dehumidification system. Renewable and Sustainable Energy Reviews, 2018. 82: p. 3236-3249.

44. Henninger, S.K., et al., New materials for adsorption heat transformation and storage. Renewable Energy, 2017. 110: p. 59-68.

45. ScienceDAILY. Pulling water from thin air. June 11, 2018]; Available from: https://www.sciencedaily.com/releases/2016/02/160224150731.htm.

46. Schemenauer, R.S. and P. Cereceda, A proposed standard fog collector for use in highelevation regions. Journal of Applied Meteorology, 1994. 33(11): p. 1313-1322.

47. Sharan, G., Dew Harvest: To Supplement Drinking Water Sources in Arid Coastal Belt of Kutch.

48. Water, W. W-Water. Available from: http://www.warkawater.org/.

49. Lindblom, J. and B. Nordell, Water production by underground condensation of humid air. Desalination, 2006. 189(1-3): p. 248-260.

50. Lawrence, M.G., The relationship between relative humidity and the dewpoint temperature in moist air: A simple conversion and applications. Bulletin of the American Meteorological Society, 2005. 86(2): p. 225-233.

51. Machine Applications Corporation, O. HUMIDITY/MOISTURE HANDBOOK.

52. Water, Z.M. Hydropanel. June 12, 2018]; Available from: https://www.zeromasswater.com/.

53. Fontus. Solar Water Bottle. Available from: http://fontus.at/products/.

54. Miller, J.E., Review of water resources and desalination technologies. Sandia national labs unlimited release report SAND-2003-0800, 2003.

55. Cargocaire, M., The Dehumidification Handbook. Munters, USA, 1990.

56. Zhang, H., et al., Concentrated solar power plants: Review and design methodology. Renewable and Sustainable Energy Reviews, 2013. 22: p. 466-481.

57. Duffie, J.A. and W. Beckman, Solar thermal engineering processes. 1980, Wiley, New York.

58. Ameel, T.A., K.G. Gee, and B.D. Wood, Performance predictions of alternative, low cost absorbents for open-cycle absorption solar cooling. Solar Energy, 1995. 54(2): p. 65-73.

59. Ge, T.S., et al., Experimental comparison and analysis on silica gel and polymer coated fin-tube heat exchangers. Energy, 2010. 35(7): p. 2893-2900.

60. Ng, K., et al., Experimental investigation of the silica gel-water adsorption isotherm characteristics. Applied Thermal Engineering, 2001. 21(16): p. 1631-1642.

61. Brunauer, S., Adsorption of Gases and Vapors [Russian translation], IL, Moscow (1948). Google Scholar: p. 389.

62. Chang, K.-S., H.-C. Wang, and T.-W. Chung, Effect of regeneration conditions on the adsorption dehumidification process in packed silica gel beds. Applied Thermal Engineering, 2004. 24(5-6): p. 735-742.

63. Wang, W., et al., An Overview of Adsorbents in the Rotary Desiccant Dehumidifier for Air Dehumidification. Drying Technology, 2013. 31(12): p. 1334-1345.

64. Küsgens, P., et al., Characterization of metal-organic frameworks by water adsorption. Microporous and Mesoporous Materials, 2009. 120(3): p. 325-330.

65. Sanders, R., Device pulls water from dry air, powered only by the sun. 2017, April. 
66. Dawoud, B. and Y. Aristov, Experimental study on the kinetics of water vapor sorption on selective water sorbents, silica gel and alumina under typical operating conditions of sorption heat pumps. International Journal of Heat and Mass Transfer, 2003. 46(2): p. 273-281.

67. Authority, H.T. The psychrometric chart and its use.

68. Van Essen, V., et al. Materials for thermochemical storage: characterization of magnesium sulfate. in Proceedings Eurosun. 2008.

69. Mistry, K.H. and S.M. Zubair, Effect of entropy generation on the performance of humidification-dehumidification desalination cycles. International Journal of Thermal Sciences, 2010. 49(9): p. 1837-1847.

70. Zubair, S.M., et al., Performance evaluation of humidification-dehumidification (HDH) desalination systems with and without heat recovery options: An experimental and theoretical investigation. Desalination, 2018. 436: p. 161-175.

71. La, D., et al., Development of a novel rotary desiccant cooling cycle with isothermal dehumidification and regenerative evaporative cooling using thermodynamic analysis method. Energy, 2012. 44(1): p. 778-791.

72. Kawamura, Y., et al., Adsorption Characteristics of Water Vapor on Gamma-Lithium Aluminate. Journal of Nuclear Science and Technology, 1992. 29(5): p. 436-444.

73. Khomane, R.B., A. Agrawal, and B.D. Kulkarni, Synthesis and characterization of lithium aluminate nanoparticles. Materials Letters, 2007. 61(23-24): p. 4540-4544.

74. Sunkara, M.K., et al., Methods for synthesizing metal oxide nanowires. 2016, Google Patents.

75. Ceyer, S.T., Dissociative chemisorption: dynamics and mechanisms. Annual Review of Physical Chemistry, 1988. 39(1): p. 479-510.

76. Andreu, N., et al., New Investigations on the Surface Reactivity of Layered Lithium Oxides. The Journal of Physical Chemistry C, 2012. 116(38): p. 20332-20341.

77. Desiccant Chart Comparisons. Available from: https://www.sorbentsystems.com/desiccants charts.html.

78. Karellas, S., et al., Energetic and exergetic analysis of waste heat recovery systems in the cement industry. Energy, 2013. 58: p. 147-156.

79. JISEA, Concentrating Solar Power and Water Issues in the U.S. Southwest.

80. Stone, B., Urban Heat and Air Pollution: An Emerging Role for Planners in the Climate Change Debate. Journal of the American Planning Association, 2005. 71(1): p. 13-25. 


\section{Appendix 1: AGGREGATES}

Several months of research were invested to research the making of solid extrudates. The following were attempted. In all cases an industrial mixer \& 'noodle' extruder was used. Weeks of time had to be spent in manually synthesizing LAO by hand, as the production was about $40 \mathrm{~g} /$ day, as each run of using the extruder required $250 \mathrm{~g}$ of the material.

We then added different binders to each batch:

- $10 \%$ Boehmite, $1 \%$ Ethyl Cellulose

- $10 \%$ Guar Gum

- $2 \%$ Ethyl Cellulose

- $2 \%$ Ethyl Cellulose and $0.1 \%$ TritonX-114

Each extrudate, diameter 1mm, (Fig 31) was dried @ 100C and then sintered at $500 \mathrm{C}$ or $900 \mathrm{C}$ (Fig 32) to burn away the binder as well as create a porous self-supported extrudate. The resulting aggregates were left overnight exposed to the atmosphere and then subject to desorption tests, using a modified syringe (Fig 33). The top of the syringe (where the needle fits) was cut out. Both ends of the syringe were closed with an aluminum mesh and taped with foil, after packing the syringe with extrudate. 


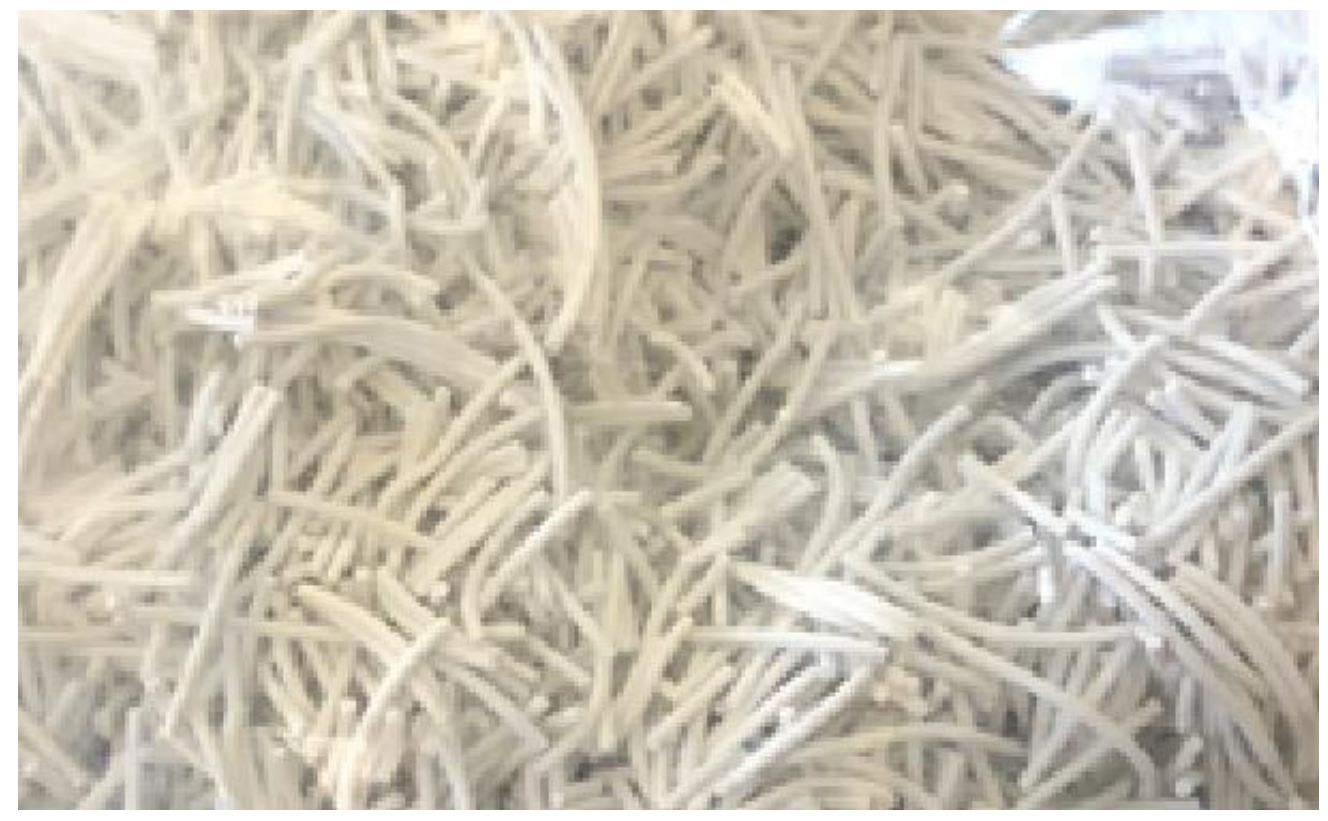

Figure 31: Dried extrudates, before sintering

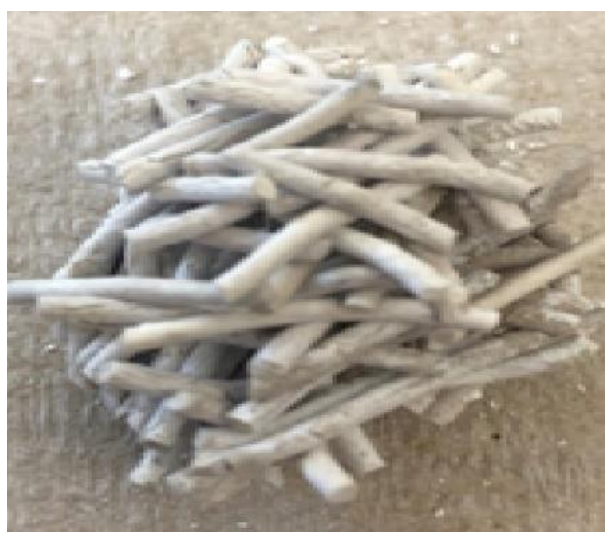

Figure 32: Sintered Extrudates 

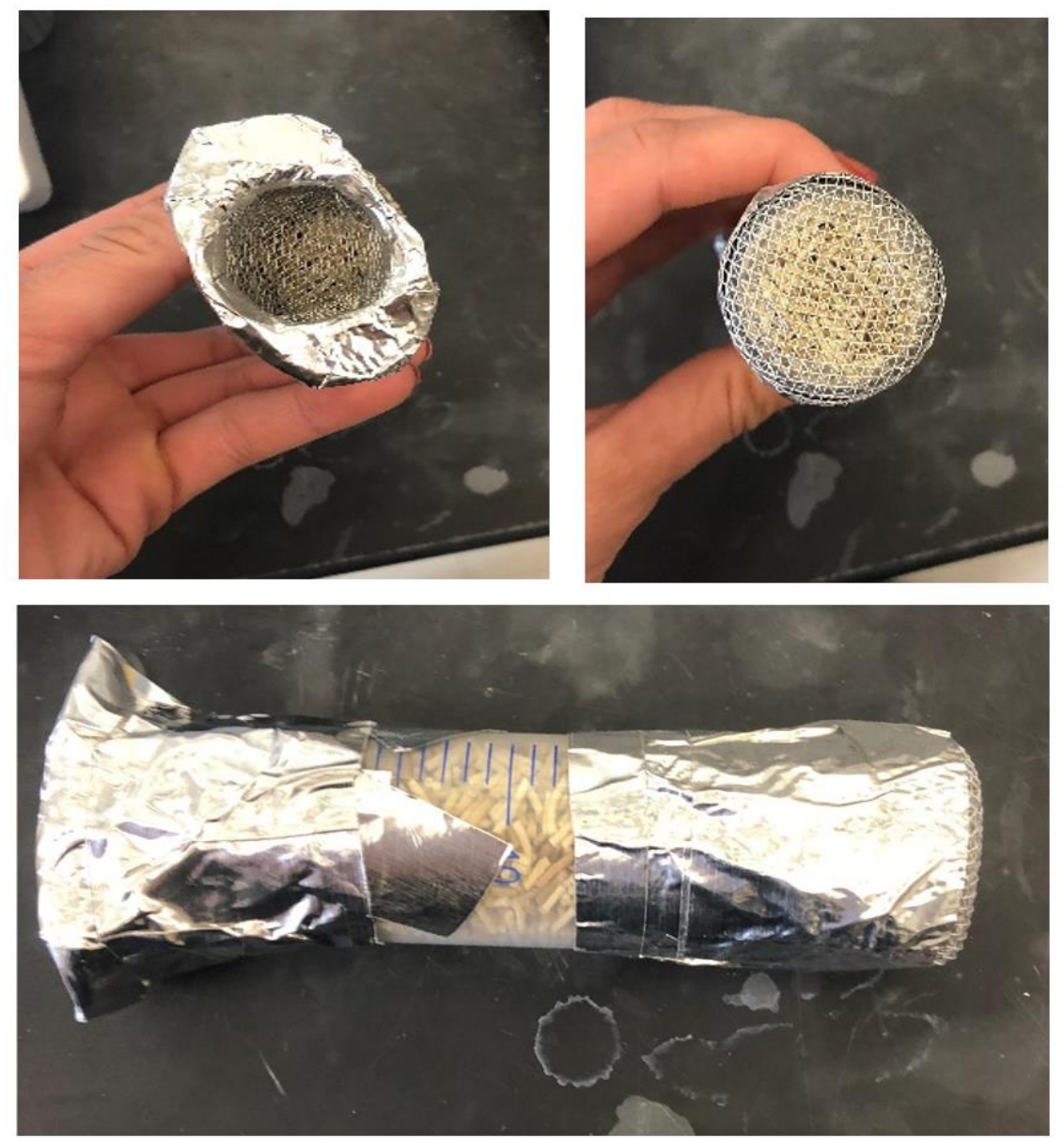

Figure 33: Test rig for the extrudates, using a modified syringe

Typical adsorption results are 'flat' $(<1 \% \mathrm{w} / \mathrm{w})$, as one example (this one was with $2 \%$ Ethyl Cellulose) in Table 8 shows (extrudate weight: $28.7368 \mathrm{~g}$ ):

\begin{tabular}{|l|r|r|r|r|r|}
\hline Status & \multicolumn{1}{|l|}{ Run 1 } & Run 2 & \multicolumn{1}{|l|}{ Run 3 } & Run 4 & \multicolumn{1}{|l|}{ Run 5 } \\
\hline $\begin{array}{l}\text { After adsorb (grams, } \\
\text { including syring) }\end{array}$ & 50.0751 & 49.9969 & 50.0142 & 50.0328 & 50.0577 \\
\hline $\begin{array}{l}\text { After desorb (grams, } \\
\text { including syring) }\end{array}$ & 49.8895 & 49.9067 & 49.9145 & 49.9463 & 49.9576 \\
\hline Wt change & 0.1856 & 0.0902 & 0.0997 & 0.0865 & 0.1001 \\
\hline $\begin{array}{l}\text { \% wt change from } \\
\text { initial sample weight }\end{array}$ & 0.65 & 0.31 & 0.35 & 0.30 & 0.35 \\
\hline
\end{tabular}

Table 9: Typical results of extrudates using the test rig of Fig 33 with 5 minutes adsorb \& 5 minutes desorb@100C, showing that the desiccant was destroyed 
We found that the adsorption capacity of LAO is destroyed in the process of making it into an aggregate. It is hypothesized that (1) using water post synthesis, causes changes in the surface of the nano wires and/or (2) that a film of carbon from the binder causes of this drop in performance.

Only the first hypothesis was tested extensively. Washed, dried and powdered samples were prepared. Tap water, deionized water, distilled water; air dry, vacuum dry, oven dry at different temperatures, post dry sintering @ 400C, 550C \& 900C were tested. In all cases TGA showed a massive drop in the characteristics, suggesting either irreversible surface chemistry changes, or the lack of excess Lithium needed in the dissociative chemisorption process. Fig 34 shows a typical TGA of one sample.

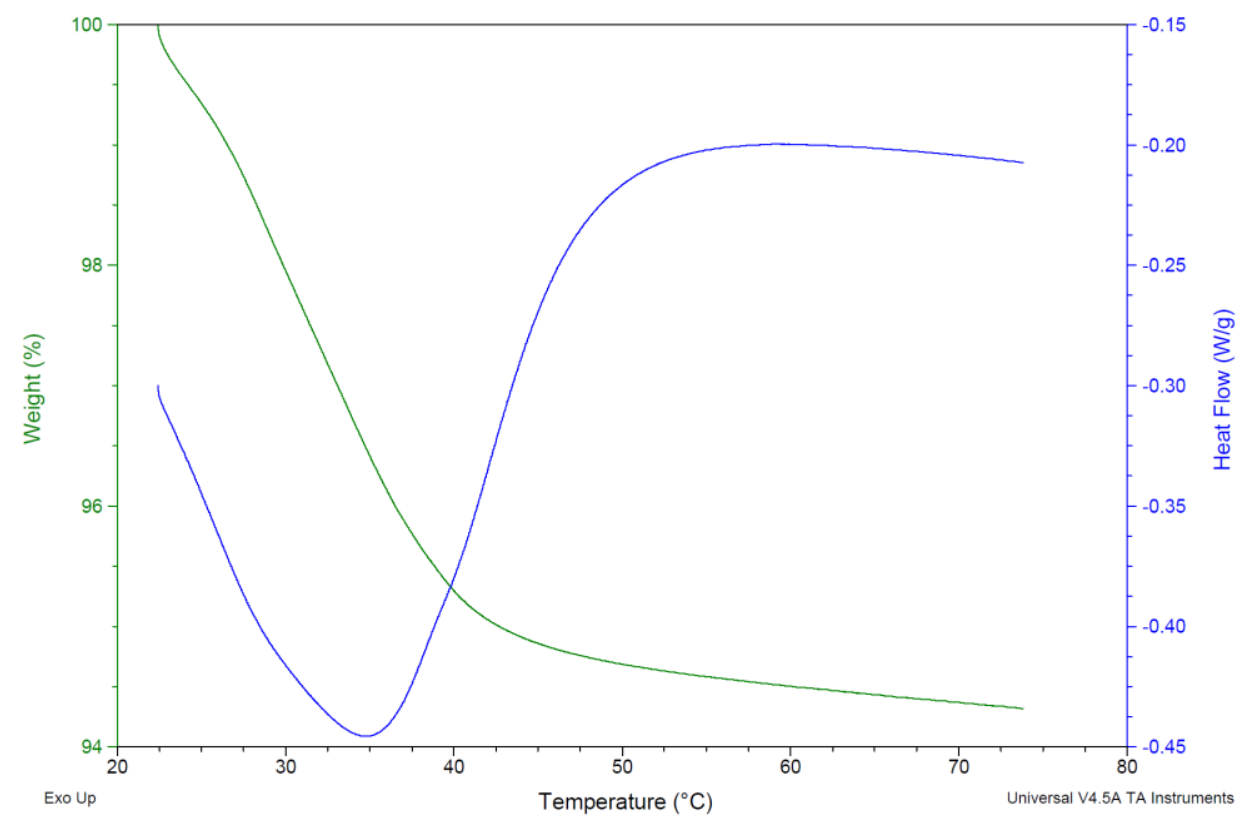

Figure 34: Typical TGA of washed sample showing a marked reduction of the desiccant's capacity 


\section{Appendix 2: WORKING PROTOTYPE}

Solar energy, as well as atmospheric temperature and humidity, are extremely dynamic and independently variable. This section details the development of a dynamic system to deliver optimal performance under wide variations in environmental conditions.

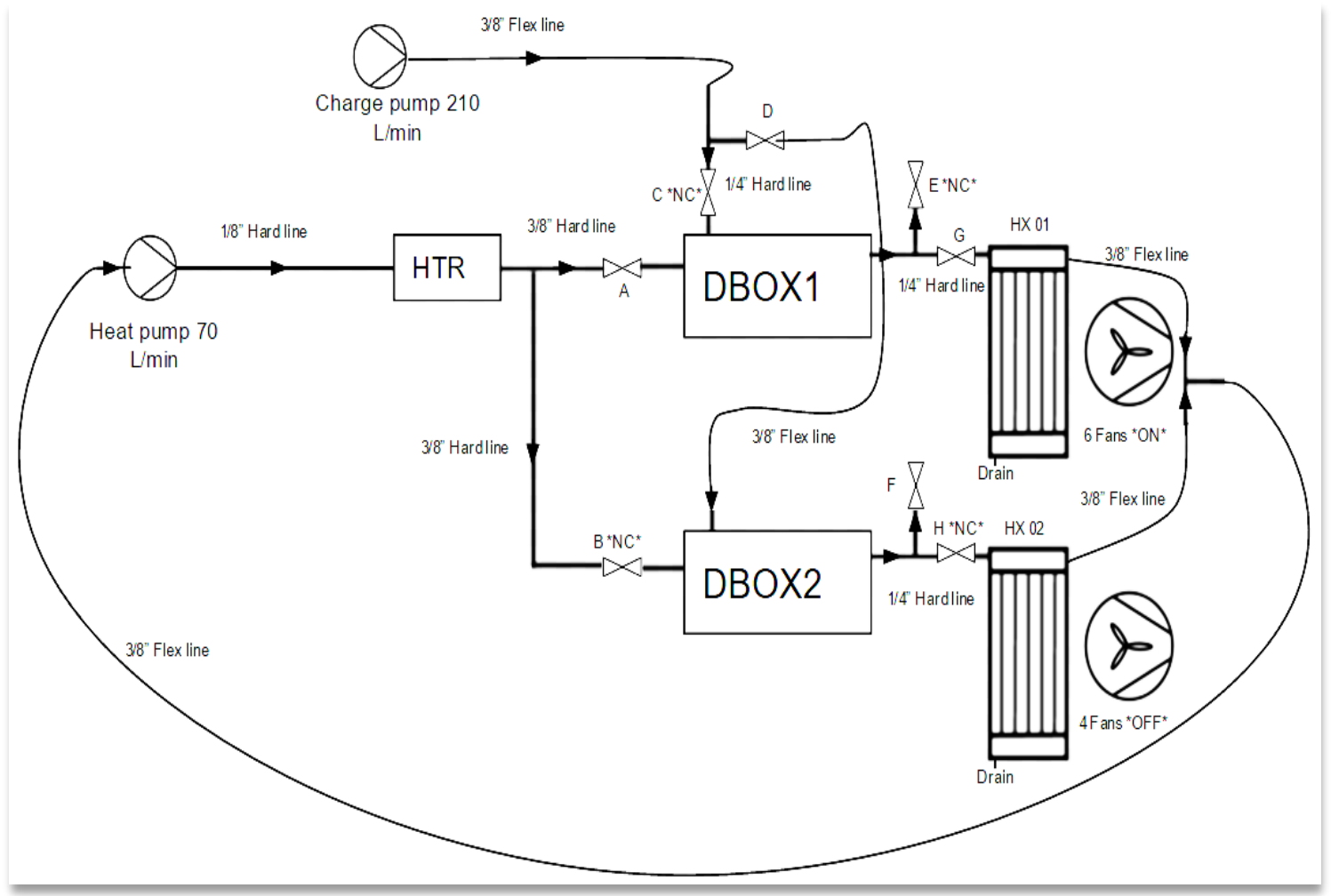

Figure 35: Schematic of the prototype

We propose a significant change from standard dehumidification / AWG systems - a completely decoupled, dynamically re-configurable, adsorption and desorption system. Fig 35 is the schematic of the prototype, Fig 36 is the solid model of the prototype while it was being built, and Fig 35 shows the actual system. 
In Fig 35, DBOX1 \& 2 are insulated chambers containing the solid desiccant. It is expected that the commercial models will have ' $n$ ' chambers. HTR is an electrically powered air heater (to be replaced eventually with solar heating), HX $01 \& 02$ are air cooled condensers - in practice it is expected to have one condenser for every ' $m$ ' ('m' $\leq$ ' $n$ ') chambers.

One or more pumps, shown as "Charge Pump", are commercially available air blowers that push filtered atmospheric air into some of the chambers - in our model, either DBOX1 or DBOX2. These chambers would be in the "adsorb" stage. Solar heated hot air is pushed through the other chambers containing saturated desiccant - in our model the other DBOX2 or DBOX1. These chambers would be in the "desorb" stage. The resulting moist air is sent into condensers whose cooling surfaces are coated with a hydrophobic substance, causing condensation of water. The collected water is pumped away or collected by gravitational force. The air leaving the intercooler is sent back to the heating system, establishing a closed air loop to improved humidity \& thermal efficiency.

With the desiccant discussed in Chapter 4 , rapid ( $\sim 3$ minutes) adsorption and desorption at low temperatures ( 80C) allows multiple cycles every day, reducing cost of device and also allows finer control of chambers to manage humidity and temperature variations. For example, with low humidity, the adsorb cycle may need to be longer that the standard $\sim 3$ minutes, or that more air is pumped through the chambers - as more pumps are pressed into service. If the weather causes the solar air heater to provide a low temperature air, the volume 
of air could be reduced, thus keeping the temperature at the level required, and a reduced number of chambers used for desorption - or the entire system could be powered down. This way active chambers are always operating at their most efficient point, and power consumption is dynamically varied based on the environmental factors.

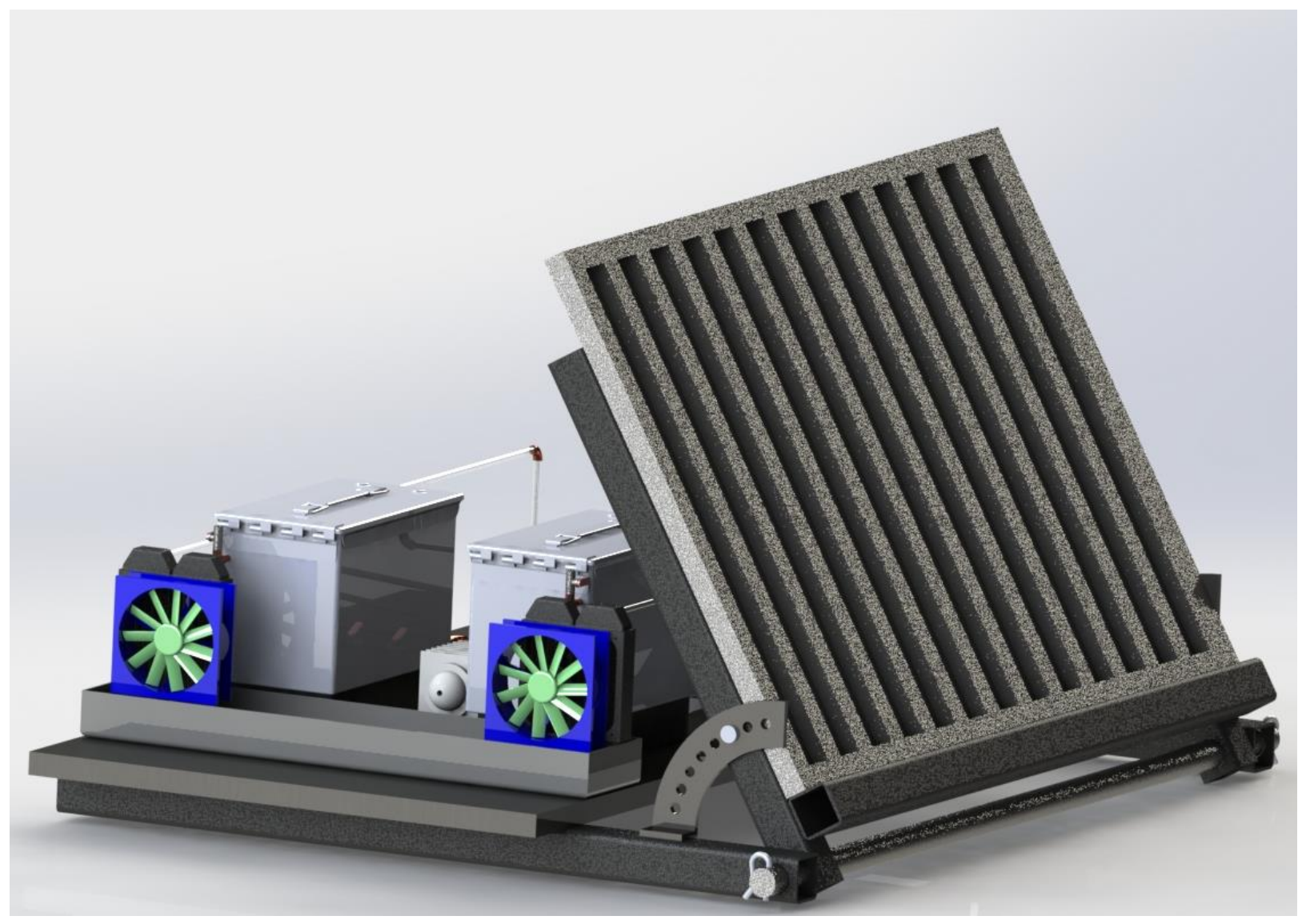

Figure 36: Solid model of prototype 


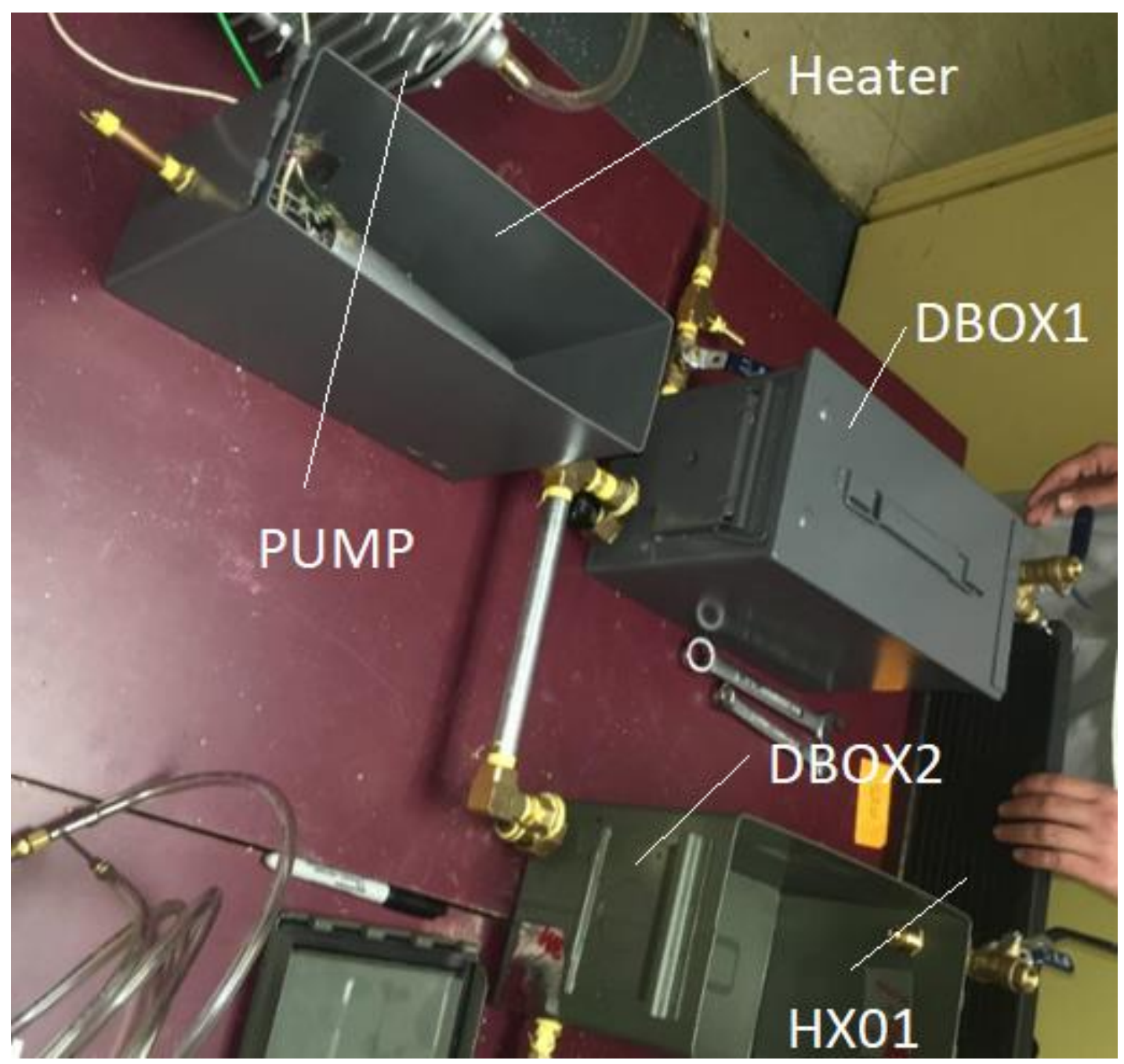

Figure 37: Prototype fabrication in progress

While the initial prototype used CPU coolers as the condenser, this was replaced by a car intercooler, as seen in Fig 38 as ' $\mathrm{HX} 01$ \& 02', in anticipation of the availability of the solid desiccant and its multiple desorb cycles. 


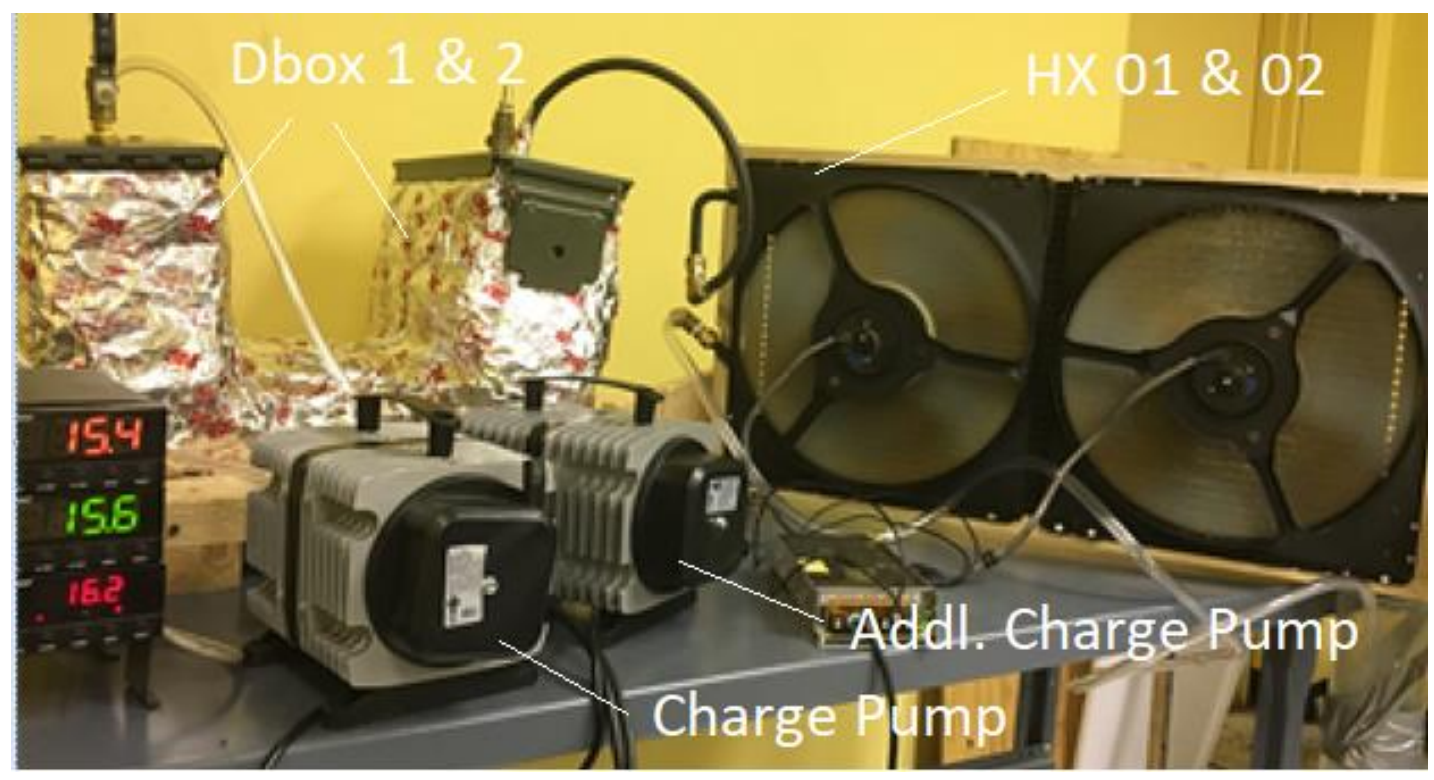

Figure 38: Use of a car intercooler as the condenser

The prototype was tested under the following ambient conditions: $60 \%$ $\mathrm{RH}, 22^{\circ} \mathrm{C}$, Silica Gel, $1.0 \mathrm{KG} /$ chamber, two chambers. Charge pump $210 \mathrm{CFM}$, heater pump 70 LPM. Table 8 has the computation and Fig 39 show the water collected over time. (This section has not been reported in the main body of the research, as this is just a test of the prototype using Silica Gel, and not of LAO aggregates) 


\begin{tabular}{|c|c|c|}
\hline Relative Humidity of Air Intake (\%) & $60.00 \%$ & Environmental factor \\
\hline Temperture of Air Intake $\left({ }^{*} \mathrm{C}\right)$ & 25.00 & Environmental factor \\
\hline Airflow Processed (m^3/hr) & 360.00 & Design decision \\
\hline Airflow Processed (ft^3/hr) & 12703.52 & \\
\hline CFM & 211.73 & \\
\hline Vapor Density of Air $\left(\mathrm{gm} / \mathrm{m}^{\wedge} 3\right)$ & 23.10 & Approx. based on temperature of the air \\
\hline Amount of Water Removed (\%) & 0.50 & $\begin{array}{l}\text { Percentage of water that is removed from } \\
\text { processed air, will be based on efficiency of } \\
\text { design }\end{array}$ \\
\hline Harvested Water (grams/hr) & 2494.30 & Based on removal of above $\%$ \\
\hline Harvested Water (L/hr) & 2.49 & \\
\hline Harvested Water (L/Day) & 59.86 & $\begin{array}{l}24 \text { hour/day opearation (as this is based on } \\
\text { electricity from the grid) }\end{array}$ \\
\hline Latent Heat (J/g) & 2441.86 & $\begin{array}{l}\text { Cubic approx. based on the temperature of the } \\
\text { air only good from }-25 \mathrm{C} \text { to } 40 \mathrm{C}\end{array}$ \\
\hline Heat Removal Rate (J/hr) & 6090747.10 & $\begin{array}{l}\text { Based on removing all latent heat from processed } \\
\text { air }\end{array}$ \\
\hline Heating rate for desorption (KJ/hr) & 6090.75 & $\begin{array}{l}\text { Based on removing all latent heat from processed } \\
\text { air }\end{array}$ \\
\hline Power needed (W) & 1691.87 & The power consumed removing latent heat \\
\hline Power for Heat Removal (KW) & 1.69 & $\begin{array}{l}\text { Power needed to heat air, approx, ignoring } \\
\text { sensible heat }\end{array}$ \\
\hline Cost of electricity KW-hr (US\$) & 0.08 & \\
\hline Cost of electricity KW-hr/Day (US\$) & 1.92 & \\
\hline Cost of Liter (US\$) & 0.03 & $\begin{array}{l}\text { Does not include power needed for blowers, } \\
\text { system and etc. }\end{array}$ \\
\hline
\end{tabular}

Table 10: Computation for the prototype, for 60 liters/day output, using electricity from the grid 


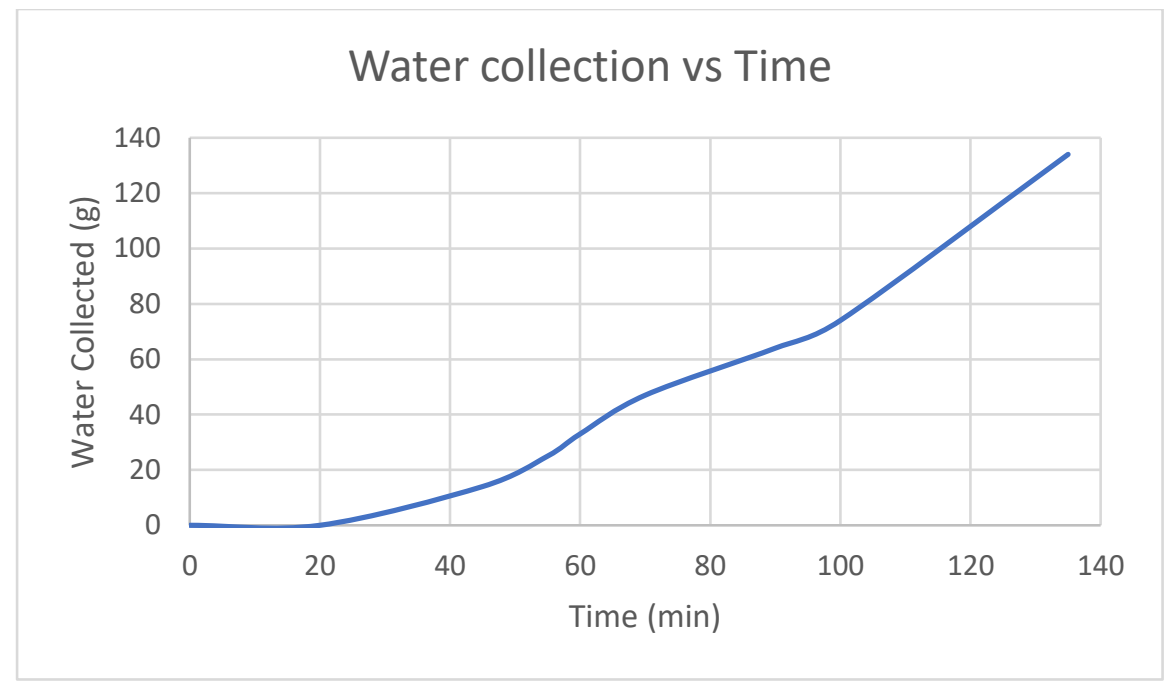

Figure 39: Water collection over time from the prototype using Silica Gel

As we stated in the introduction to this section, we can see how with multiple desiccant chambers, we can control the numbers that are in adsorption \& desorption - all based on the humidity in the environment, ambient \& condenser temperatures and solar insolation available for heating - ensuring that those chambers that are operating are doing so at their maximum operating efficiencies. The condensers and blowers would similarly be multiple units (for example the "Addl. Charge Pump" in Fig. 38), thus ensuring the highest energy and system efficiency.

We believe that this is a unique design approach, one not seen in the literature, and is suitable for one or more design patents.

An interesting source of thermal energy is from waste heat from industrial plants that otherwise has little exergy left [78]. With a AWG system using this low temperature desiccant, water can be cheaply extracted from air, which often is a 
critical input for most industries [79] and reducing industrial heat pollution [80] at the same time. 


\section{CURRICULUM VITAE}

Name: Sunil Gupta

Address: Department of Mechanical Engineering

J.B.Speed School of Engineering

University of Louisville

Louisville, KY 40292

Education: M.S., Mechanical Engineering

University of Louisville

Louisville, KY 40292

B.E., Mechanical Engineering

Bangalore University

Karnataka, INDIA 560056 